\title{
LOCALIZATIONS FOR QUIVER HECKE ALGEBRAS
}

\author{
MASAKI KASHIWARA, MYUNGHO KIM, SE-JIN OH, AND EUIYONG PARK \\ In Memory of Professor Bertram Kostant
}

\begin{abstract}
In this paper, we provide a generalization of the localization procedure for monoidal categories developed in [12] by Kang-Kashiwara-Kim by introducing the notions of braiders and a real commuting family of braiders. Let $R$ be a quiver Hecke algebra of arbitrary symmetrizable type and $R$-gmod the category of finitedimensional graded $R$-modules. For an element $w$ of the Weyl group, $\mathscr{C}_{w}$ is the subcategory of $R$-gmod which categorifies the quantum unipotent coordinate algebra $A_{q}(\mathfrak{n}(w))$. We construct the localization $\widetilde{\mathscr{C}}_{w}$ of $\mathscr{C}_{w}$ by adding the inverses of simple modules $\mathrm{M}\left(w \Lambda_{i}, \Lambda_{i}\right)$ which correspond to the frozen variables in the quantum cluster algebra $A_{q}(\mathfrak{n}(w))$. The localization $\widetilde{\mathscr{C}}_{w}$ is left rigid and it is conjectured that $\widetilde{\mathscr{C}}_{w}$ is rigid.
\end{abstract}

\section{Contents}

Introduction 2

1. Preliminaries 7

1.1. Quantum groups 7

1.2. Quiver Hecke algebras 9

1.3. Cyclotomic quiver Hecke algebras 12

1.4. The categories $\mathscr{C}_{w} \quad 14$

Date: December 28, 2020.

2010 Mathematics Subject Classification. 18D10, 16D90, 81R10.

Key words and phrases. Categorification, Localization, Monoidal category, Quantum unipotent coordinate ring, Quiver Hecke algebra.

The research of M. Ka. was supported by Grant-in-Aid for Scientific Research (B) 15H03608, Japan Society for the Promotion of Science.

The research of M. Kim was supported by the National Research Foundation of Korea(NRF) Grant funded by the Korea government(MSIP) (NRF-2017R1C1B2007824).

The research of S.-j. Oh was supported by the Ministry of Education of the Republic of Korea and the National Research Foundation of Korea (NRF-2019R1A2C4069647).

The research of E. P. was supported by the National Research Foundation of Korea(NRF) Grant funded by the Korea Government(MSIP)(NRF-2017R1A1A1A05001058). 
1.5. Monoidal categories 16

2. Localizations 20

2.1. Real commuting family of braiders 20

2.2. Localizations 23

2.3. Graded cases 32

3. Affinizations and R-matrices 37

3.1. Affinizations 37

3.2. R-matrices 41

3.3. Determinantial modules 47

4. Braiders in $R$-gmod $\quad 52$

4.1. Non-degenerate braiders $\quad 52$

4.2. Localization $\quad 55$

4.3. Left duals $\quad 58$

5. Localization of $\mathscr{C}_{w} \quad 65$

5.1. Graded braiders associated with a Weyl group element 65

5.2. Rank-one case 68

5.3. Left rigidity of $\tilde{\mathscr{C}}_{w} \quad 69$

$\begin{array}{ll}\text { References } & 74\end{array}$

\section{INTRODUCTION}

Khovanov-Lauda and Rouquier independently introduced a family of $\mathbb{Z}$-graded associative algebras $R(\beta)$ over a commutative ring $\mathbf{k}$, called the quiver Hecke algebras, in order to categorify the half of a quantum group $U_{q}(\mathfrak{g})([18,22])$. For an $R(\beta)$ module $M$ and an $R(\gamma)$-module $N$, one can form an $R(\beta+\gamma)$-module $M \circ N$ from the $R(\beta) \otimes R(\gamma)$-module $M \otimes N$, called the convolution product, which yields monoidal category structures on the direct sum $R$-proj of the categories of finitely generated projective graded $R(\beta)$-modules and the direct sum $R$-gmod of the categories of finite dimensional graded $R(\beta)$-modules. It turns out that the Grothendieck rings $K(R$-proj) and $K(R$-gmod $)$ are isomorphic to the half of quantum group $U_{q}^{-}(\mathfrak{g})$ and to its dual $U_{q}^{-}(\mathfrak{g})^{*}$, respectively. Moreover, when $\mathfrak{g}$ is symmetric and $\mathbf{k}$ is a field of characteristic zero, the set of classes of indecomposable projective graded modules and the set of classes of simple graded modules correspond to the lower and upper global basis, respectively $([23,25])$.

The monoidal category $R$-gmod has an interesting connection with quantum affine algebras via KLR-type quantum Schur-Weyl duality. In [12], a family of functors from 
$R$-gmod to the categories of finite-dimensional modules over quantum affine algebras was introduced. Briefly speaking, for a given family of simple modules over the quantum affine algebra that we are interested in, we have a procedure to obtain a quiver $\Gamma$ from the family and a functor $\mathcal{F}$ from $R^{\Gamma}$-gmod to the category of modules over the quantum affine algebra, where $R^{\Gamma}$ is the quiver Hecke algebra corresponding to the symmetric Kac-Moody algebra associated with the underlying graph of the quiver $\Gamma$. Among those functors, the case when the quantum affine algebra is of type $A_{N-1}^{(1)}$ and the family is given as all the successive $q^{ \pm 2}$-shifts of the first fundamental representation $V\left(\varpi_{1}\right)$, was studied in detail. In this case, the quiver Hecke algebra is of type $A_{\infty}$ which is denoted by $R^{A_{\infty}}$. Set $\mathcal{A}:=R^{A_{\infty}}$-gmod. Then the functor $\mathcal{F}$ factors through the quotient category $\mathcal{A} / \mathcal{S}_{N}$, where $\mathcal{S}_{N}$ denotes the kernel of the functor $\mathcal{F}$. But still there are rather big differences between the category $\mathcal{A} / \mathcal{S}_{N}$ and the full subcategory consisting of images of $\mathcal{F}$, denoted by $\mathcal{C}_{A_{N-1}^{(1)}}^{0}$ : there is an infinite family of simples in $\mathcal{A} / \mathcal{S}_{N}$ which are isomorphic to the unit object (trivial representation) of $\mathcal{C}_{A_{N-1}^{(1)}}^{0}$ under $\mathcal{F}$, and the latter is a rigid monoidal category, i.e., every object has left and right duals, whereas the former is not. In an effort to obtain a more similar category to $\mathcal{C}_{A_{N-1}^{(1)}}^{0}$, a localization of the category $\mathcal{A} / S_{N}$ by a commuting family of central objects was introduced in [12]. More precisely, a monoidal category $\mathcal{T}_{N}$ and a functor $\Phi: \mathcal{A} / \mathcal{S}_{N} \rightarrow \mathcal{T}_{N}$ were constructed such that the functor $\mathcal{F}$ factors through $\Phi$ and the simple objects in $\mathcal{A} / \mathcal{S}_{N}$ which correspond to the trivial representation under $\mathcal{F}$ become isomorphic to the unit object in $\mathcal{T}_{N}$ under $\Phi$. Moreover it turned out that the category $\mathcal{T}_{N}$ is a rigid monoidal category. One of key observations was that the family considered above forms a commuting family of central objects and this condition is enough to construct a larger category on which all the objects in the family can be inverted. Note that the construction in [12, Appendix A] was done in an abstract setting: i.e., for an arbitrary monoidal category $\mathcal{T}$ with a commuting family of central objects, one obtains a localization of $\mathcal{T}$ with desired properties.

Since the application of the localization procedure to the category $\mathcal{A} / \mathcal{S}_{N}$ was quite successful, it is natural to pursue further along this line with other monoidal categories coming from $R$-gmod. For example, for each element $w$ in the Weyl group of $\mathfrak{g}$, there is an interesting monoidal subcategory $\mathscr{C}_{w}$ of $R$-gmod whose Grothendieck ring is isomorphic to the quantum unipotent coordinate ring $A_{q}(\mathfrak{n}(w))$. Moreover the category $\mathscr{C}_{w}$ reflects the quantum cluster algebra structure on $A_{q}(\mathfrak{n}(w))$ in an essential way: i.e., all the cluster monomials belong to the set of classes of simple modules in $\mathscr{C}_{w}([14])$. Note that in the quantum cluster algebra $A_{q}(\mathfrak{n}(w))$, the frozen cluster variables are not invertible, whereas there are several works considering the version of unipotent quantum 
coordinate ring with invertible frozen variables (for example, see [20, 21]). Hence it is desirable to have a localization of the category $\mathscr{C}_{w}$ on which the modules corresponding to the frozen variables are invertible. But the procedure in $[12$, Appendix A] is not directly applicable to this case because the simple modules of $\mathscr{C}_{w}$ corresponding to the frozen variables may not form a commuting family of central objects.

The first goal of this paper is to provide a generalization of the localization procedure developed in [12], which is applicable to more general cases. We introduce a braider and a real commuting family of braiders which generalize a central object and a commuting family of central objects in a broad sense. Let $\mathcal{T}$ be a $\mathbf{k}$-linear monoidal category with the tensor product $\otimes$ and a unit object 1. A pair $\left(C, R_{C}\right)$ of an object $C$ and a natural transformation $R_{C}:(C \otimes-) \rightarrow(-\otimes C)$ in $\mathcal{T}$ is called a (left) braider of $\mathcal{T}$, when $R_{C}$ is compatible with the tensor product and the unit object (see (2.1)). Note that we do not require that $R_{C}(X)$ are isomorphisms which means that $C$ may not be central. We call a family $\left\{\left(C_{i}, R_{C_{i}}\right)\right\}_{i \in I}$ of braiders in $\mathcal{T}$ a real commuting family if $R_{C_{i}}\left(C_{i}\right) \in \mathbf{k}^{\times}$id $_{C_{i} \otimes C_{i}}$ and $R_{C_{j}}\left(C_{i}\right) \circ R_{C_{i}}\left(C_{j}\right) \in \mathbf{k}^{\times} \mathrm{id}_{C_{i} \otimes C_{j}}$, which is a key difference from a commuting family of central objects. In the case of a commuting family of central objects, the morphisms $R_{C_{i}}\left(C_{i}\right)$ and $R_{C_{j}}\left(C_{i}\right) \circ R_{C_{i}}\left(C_{j}\right)$ should be the identities. This difference seems to be small but important and essential (see Example 4.7). One of our main theorems is the existence and the uniqueness of localization of the monoidal category $\mathcal{T}$ via the real commuting family $\left\{\left(C_{i}, R_{C_{i}}\right)\right\}_{i \in I}$ of braiders (Theorem 2.7): there exists a monoidal category $\widetilde{\mathcal{T}}$ and a monoidal functor $\Upsilon: \mathcal{T} \rightarrow \widetilde{\mathcal{T}}$ such that the object $\Upsilon\left(C_{i}\right)$ is invertible in $\widetilde{\mathcal{T}}$ and the morphisms $\Upsilon\left(R_{C_{i}}(X)\right): \Upsilon\left(C_{i} \otimes X\right) \rightarrow \Upsilon\left(X \otimes C_{i}\right)$ are isomorphisms in $\widetilde{\mathcal{T}}$ for any $i \in I$ and $X \in \mathcal{T}$. Moreover, the pair $(\widetilde{\mathcal{T}}, \Upsilon)$ is universal (an initial object) among those satisfying the above two conditions. A part of this paper is devoted to define the compositions and the tensor products of morphisms in $\widetilde{\mathcal{T}}$ so that $\widetilde{\mathcal{T}}$ becomes a monoidal category. Since our main motivation is to construct localizations of monoidal categories arising from $R$-gmod, we also developed the localization for graded cases: assume that $\mathcal{T}$ is a k-linear monoidal category with a decomposition $\mathcal{T}=\bigoplus_{\lambda \in \Lambda} \mathcal{T}_{\lambda}$ for some abelian group $\Lambda$ such that $\mathbf{1} \in \mathcal{T}_{0}$ and $\otimes$ induces a bifunctor $\mathcal{T}_{\lambda} \times \mathcal{T}_{\mu} \rightarrow \mathcal{T}_{\lambda+\mu}$ for any $\lambda, \mu \in \Lambda$. We further assume that there is an invertible central object $q$ in $\mathcal{T}_{0}$. Then we introduce a notion of graded braiders $\left(C, R_{C}, \phi\right)$, which roughly means that $\left(C, R_{C}\right)$ is a braider with an abelian group homomorphism $\phi: \Lambda \rightarrow \mathbb{Z}$ such that $R_{C}(X): C \otimes X \rightarrow q^{\phi(\lambda)} \otimes X \otimes C$ for $X \in \mathcal{T}_{\lambda}$. These are modelled on simple modules in $R$-gmod and homogeneous homomorphisms between convolution products. Then by a careful study on the degrees of morphisms, we obtain a localization of $\mathcal{T}$ with analogous properties as ungraded cases. 
Our second goal is to apply the localization procedure we have developed to subcategories of $R$-gmod. Assume that $R$ is a quiver Hecke algebra of arbitrary symmetrizable type. A distinguished feature of the monoidal category $R$-gmod is that every simple object $M$ admits a structure of a non-degenerate graded braider $\left(M, R_{M}, \phi\right)$, which is unique in some sense (Lemma 4.3). Thus one can consider various localizations of subcategories of $R$-gmod. We are more interested in the cases that the real commuting family of graded braiders in a subcategory $\mathscr{C}$ of $R$-gmod is originated from a family of real simple modules admitting affinizations, because such a case enjoys more pleasant properties out of $R$-matrices. For a quiver Hecke algebra of arbitrary type, the notion of affinization was developed in [16] in order to define the $R$-matrices, which is the distinguished nonzero homomorphism $\mathbf{r}_{M, N}: q^{\Lambda(M, N)} M \circ N \rightarrow N \circ M$ for simple $R$-modules $M$ and $N$ such that one of them admits an affinization. Let $M$ be a real simple $R$-module with an affinization. Then we investigate the non-degenerate graded braider structure $\left(M, R_{M}, \phi\right)$, which is closely related to the R-matrices. Proposition 4.4 tells that the morphism $R_{M}(N)$ for a simple module $N$ is either zero or equal to $\mathbf{r}_{M, N}$ up to a constant multiple, and Lemma 4.6 gives a criterion to check when given non-degenerate braiders $\left(S_{a}, R_{S_{a}}, \phi\right)$ form a real commuting family of graded braiders. It is shown in Proposition 4.8 that if $\left(C_{a}, R_{C_{a}}, \phi_{a}\right)_{a \in A}$ is a real commuting family of non-degenerate graded braiders in $\mathscr{C}$ such that every $C_{a}$ admits an affinization, then the localization functor $\Phi: \mathscr{C} \rightarrow \tilde{\mathscr{C}}$ sends a simple $S$ to a simple or zero.

Thus it is important to determine whether a given simple module admits an affinizations or not. Note that if the quiver Hecke algebra $R$ is of symmetric type, then there is a natural affinization for any module in $R$-gmod ([12]). But in general, finding affinizations of a given $R$-module is a nontrivial problem. In this paper, we prove that a special family of simple modules, called the determinantial modules, admit affinizations (Theorem 3.26). The determinantial module $\mathrm{M}(\lambda, \mu)$ associated with a pair of weights $\lambda, \mu$ in the Weyl group orbit of a dominant integral weight of $\mathfrak{g}$ is a simple $R$-module corresponding to the unipotent quantum minors $D(\lambda, \mu)$, which is a certain element in the upper global basis ([15]). To prove existence of affinizations of determinantial modules, we introduce and investigate the polynomials $\chi_{i}(M), \mathfrak{e}_{i}(M)$ and $\mathfrak{e}_{i}^{*}(M)$ associated with an $R$-module $M$ (Definition 3.4). Note that $\mathfrak{e}_{i}(\widehat{L})$ and $\mathfrak{e}_{i}^{*}(\widehat{L})$ can be viewed as lifts of $\varepsilon_{i}(L)$ and $\varepsilon_{i}^{*}(L)$ when $\widehat{L}$ is an affinization of a simple module $L$. Then we consider the determinantial module $\mathrm{M}\left(\lambda, \mu ; a_{\Lambda}\right)$ using the cyclotomic quiver Hecke algebra $R_{A}^{a_{\Lambda}}$ over the polynomial ring $A=\mathbf{k}[z]$, and prove that $\mathrm{M}\left(\lambda, \mu ; a_{\Lambda}\right)$ is an affinization of $\mathrm{M}(\lambda, \mu)$ by studying the inductive formula for $\mathfrak{e}_{i}\left(\mathrm{M}\left(\lambda, \Lambda ; a_{\Lambda}\right)\right)$ given in Proposition 3.25. Recall that the ring $A_{q}(\mathfrak{n}(w))$ has a set of generators consisting of some unipotent quantum minors. In particular, the determinantial modules $\left\{\mathrm{C}_{i}:=\mathrm{M}\left(w \Lambda_{i}, \Lambda_{i}\right)\right\}_{i \in I}$ belong to the 
category $\mathscr{C}_{w}$ and they correspond to the frozen variables of the quantum cluster algebra $A_{q}(\mathfrak{n}(w))$. Proposition 5.1 tells that $\left\{\left(\mathrm{C}_{i}, R_{\mathrm{C}_{i}}, \phi_{i}\right)\right\}_{i \in I}$ is a real commuting family of non-degenerate graded braiders in the category $\mathscr{C}_{w}$, which actually are central in $\mathscr{C}_{w}$ (Theorem 5.2). Thus there exists a localization $\tilde{\mathscr{C}}_{w}$ of $\mathscr{C}_{w}$ by the family $\left\{\left(\mathrm{C}_{i}, R_{\mathrm{C}_{i}}, \phi_{i}\right)\right\}_{i \in I}$ with the canonical functor $\Phi: \mathscr{C}_{w} \rightarrow \widetilde{\mathscr{C}}_{w}$, which was one of our primary goals. It follows that the Grothendieck ring $K\left(\widetilde{\mathscr{C}}_{w}\right)$ is a localization of the quantum cluster algebra $A_{q}(\mathfrak{n}(w))$ at the set of frozen variables.

In the remaining part of the paper, we investigate rigidity for the localization $\widetilde{\mathscr{C}}_{w}$. Surprisingly the localization $\widetilde{\mathscr{C}}_{w}$ is left rigid, i.e., every object of $\widetilde{\mathscr{C}}_{w}$ has a left dual, and every simple objects of $\widetilde{\mathscr{C}}_{w}$ has a right dual. We conjecture that $\widetilde{\mathscr{C}}_{w}$ is rigid, i.e., every object of $\widetilde{\mathscr{C}}_{w}$ has left and right dual (Conjecture 5.12). We first show that every simple object in $\widetilde{\mathscr{C}}_{w}$ has a right dual (Theorem 5.7 ) by using the T-system appeared in $[9$, Proposition 3.2] crucially. Then, we use the localization $\widetilde{\mathscr{R}}$ of the whole category $R$-gmod via the same family $\left\{\left(\mathrm{C}_{i}, R_{\mathrm{C}_{i}}, \phi_{i}\right)\right\}_{i \in I}$. Note that the generalization from the central objects to the braiders is essential for the localization $\widetilde{\mathscr{R}}$, since the braiders $\left(\mathrm{C}_{i}, R_{\mathrm{C}_{i}}\right)$ are not central in $\widetilde{\mathscr{R}}$ anymore. There are some advantage of working on this auxiliary category: every finite-dimensional quotient of the affinization of $L(i)$ has a left dual in the category $\widetilde{\mathscr{R}}$ (Theorem 4.12 ). Since any nonzero module in $R$-gmod is a cokernel of a homomorphism between some direct sums of convolution products of such quotients, it follows that every object in $\widetilde{\mathscr{R}}$ has a left dual. Moreover, those left duals belong to the category $\widetilde{\mathscr{C}}_{w}$ (Theorem 5.8). We next prove that the functor $\widetilde{\mathscr{C}}_{w} \rightarrow \widetilde{\mathscr{R}}$ induced from the embedding $\mathscr{C}_{w} \rightarrow R$-gmod is actually an equivalence of categories (Theorem 5.9), so that the left rigidity of $\widetilde{\mathscr{C}}_{w}$ follows. When $\mathfrak{g}$ is of finite type and $w$ is the longest element $w_{0}$, we prove that Conjecture 5.12 is true, i.e., $\widetilde{\mathscr{C}}_{w_{0}}$ is rigid (Theorem 5.13). Moreover, the functor $\mathscr{D}^{-1}$ given by taking the left dual is 6-periodic on the set of simple objects in this case (Proposition 5.14).

Finally we remark that the rigidity of the localization $\widetilde{\mathscr{C}}_{w}$ is closely related with the twist automorphism on the coordinate ring $\mathbb{C}\left[N^{w}\right]$ of the unipotent cells and its quantum analogues (see $[2,3,4,10,8,20]$ ). The twist automorphism was introduced in order to give formulas for the inverses of parameterizations of Lusztig's totally positive parts of unipotent subgroups and Schubert varieties $([2,4])$. When $\mathfrak{g}$ is of symmetric type, the additive categorification of the twist automorphism was given in [8] using the Sykes's functor $\Omega_{w}$ on the Frobenius subcategory associated with $w$ of the category of representations of a preprojective algebra. It is well-known that the functor $\Omega_{w}$ is 6 -periodic when $\mathfrak{g}$ is of finite type and $w$ is the longest element $([1,6])$. Thus the twist automorphism also has a periodicity in those cases. In [20], Kimura-Oya 
constructed the quantum twist automorphism on the algebra $A_{q}\left[N_{-}^{w}\right]$ which is isomor-

phic to $\mathbb{Q}(q) \otimes_{\mathbb{Z}\left[q^{ \pm 1}\right]} K\left(\widetilde{\mathscr{C}}_{w}\right)$. They showed that the specialization at $q=1$ recovers the twist automorphism on $\mathbb{C}\left[N^{w}\right]$ and it is 6 -periodic when $\mathfrak{g}$ is of finite type and $w$ is the longest element. Moreover, it preserves the upper global basis. Based on these properties, they asked whether the quantum twist automorphism can be categorified by using finite-dimensional modules over quiver Hecke algebra or not ([20, Remark 7.27]). The answer is affirmative, because the quantum twist automorphism corresponds to the map induced from the left dual functor $\mathscr{D}^{-1}$ at the Grothendieck group level, up to the antiautomorphism which fixes classes of simple objects (see the formula in [20, Theorem 6.1]). In this sense, we may say that the duality functor on $\widetilde{\mathscr{C}}_{w}$ is a monoidal categorification of the quantum twist automorphism in [20].

This paper is organized as follows: In Section 1, we recall quantum groups, quiver Hecke algebras, the category $\mathscr{C}_{w}$ and some generalities on monoidal categories. In Section 2, we develop the localization of monoidal categories via a real commuting family of braiders. In Section 3, we recall the affinizations and $R$-matrices of quiver Hecke algebras and prove that the determinantial modules admit affinizations. In Section 4 , we study the braiders in $R$-gmod and the localization of subcategories of $R$-gmod in detail. In Section 5 , we show that the localization $\widetilde{\mathscr{C}}_{w}$ of the category $\mathscr{C}_{w}$ is left rigid and every simple objects in it has a right dual. In finite type $\mathfrak{g}$ with the longest element $w_{0}$ case, we showed that $\widetilde{\mathscr{C}}_{w_{0}}$ is rigid and the duality functors are 6 -periodic.

\section{Acknowledgments}

We thank Yoshiyuki Kimura for informing us his result with Hironori Oya and for fruitful communications, and the first author thank Christof Geiß and Bernard Leclerc for fruitful discussion. The second, third and fourth authors gratefully acknowledge for the hospitality of RIMS (Kyoto University) during their visit in 2018. The authors also would like to thank the anonymous reviewer for valuable comments.

\section{Preliminaries}

\subsection{Quantum groups.}

Let $I$ be an index set. A Cartan datum $\left(\mathrm{A}, \mathrm{P}, \Pi, \Pi^{\vee},(\cdot, \cdot)\right)$ consists of

(i) a free abelian group $\mathrm{P}$, called the weight lattice,

(ii) $\Pi=\left\{\alpha_{i} \mid i \in I\right\} \subset \mathrm{P}$, called the set of simple roots,

(iii) $\Pi^{\vee}=\left\{h_{i} \mid i \in I\right\} \subset \mathrm{P}^{\vee}:=\operatorname{Hom}(\mathrm{P}, \mathbb{Z})$, called the set of simple coroots,

(iv) a $\mathbb{Q}$-valued symmetric bilinear form $(\cdot, \cdot)$ on $\mathrm{P}$,

which satisfy 
(a) $\left(\alpha_{i}, \alpha_{i}\right) \in 2 \mathbb{Z}_{>0}$ for $i \in I$,

(b) $\left\langle h_{i}, \lambda\right\rangle=\frac{2\left(\alpha_{i}, \lambda\right)}{\left(\alpha_{i}, \alpha_{i}\right)}$ for $i \in I$ and $\lambda \in \mathrm{P}$,

(c) $\mathrm{A}:=\left(\left\langle h_{i}, \alpha_{j}\right\rangle\right)_{i, j \in I}$ is a generalized Cartan matrix, i.e., $\left\langle h_{i}, \alpha_{i}\right\rangle=2$ for any $i \in I$ and $\left\langle h_{i}, \alpha_{j}\right\rangle \in \mathbb{Z}_{\leq 0}$ if $i \neq j$,

(d) $\Pi$ is a linearly independent set,

(e) for each $i \in I$, there exists $\Lambda_{i} \in \mathrm{P}$ such that $\left\langle h_{j}, \Lambda_{i}\right\rangle=\delta_{i j}$ for any $j \in I$.

Let $\Delta$ (resp. $\Delta_{+}, \Delta_{-}$) be the set of roots (resp. positive roots, negative roots). We set $\mathrm{P}_{+}:=\left\{\lambda \in \mathrm{P} \mid\left\langle h_{i}, \lambda\right\rangle \geq 0\right.$ for $\left.i \in I\right\}, \mathrm{Q}=\bigoplus_{i \in I} \mathbb{Z} \alpha_{i}$, and $\mathrm{Q}_{+}=\sum_{i \in I} \mathbb{Z}_{\geq 0} \alpha_{i}$, and write $\operatorname{ht}(\beta)=\sum_{i \in I} k_{i}$ for $\beta=\sum_{i \in I} k_{i} \alpha_{i} \in \mathrm{Q}_{+}$. For $i \in I$, we define

$$
s_{i}(\lambda)=\lambda-\left\langle h_{i}, \lambda\right\rangle \alpha_{i} \quad \text { for } \lambda \in \mathrm{P},
$$

and $\mathrm{W}$ is the subgroup of Aut(P) generated by $\left\{s_{i}\right\}_{i \in I}$. For $w, v \in \mathbf{W}$, we write $w \geq v$ if there exists a reduced expression of $v$ which appears in a subexpression of a reduced expression of $w$.

For $\lambda, \mu \in \mathrm{P}$, we write $\lambda \preceq \mu$ if there exists a sequence of real positive roots $\beta_{k}$ $(1 \leq k \leq \ell)$ such that $\lambda=s_{\beta_{\ell}} \cdots s_{\beta_{1}} \mu$ and $\left(\beta_{k}, s_{\beta_{k-1}} \cdots s_{\beta_{1}} \mu\right)>0$ for $1 \leq k \leq \ell$. Note that, for $\Lambda \in \mathrm{P}_{+}$and $\lambda, \mu \in \mathrm{W} \Lambda, \lambda \preceq \mu$ holds if and only if there exist $w, v \in \mathrm{W}$ such that $\lambda=w \Lambda, \mu=v \Lambda$ and $v \leq w$.

Let $U_{q}(\mathfrak{g})$ be the quantum group associated with $\left(\mathrm{A}, \mathrm{P}, \Pi, \Pi^{\vee},(\cdot, \cdot)\right)$, which is an associative algebra over $\mathbb{Q}(q)$ generated by $e_{i}, f_{i}(i \in I)$ and $q^{h}\left(h \in \mathrm{P}^{\vee}\right)$ with certain defining relations (see [7, Chap. 3] for details). Let $*$ be the $\mathbb{Q}(q)$-antiautomorphism of $U_{q}(\mathfrak{g})$ defined by

$$
e_{i}^{*}=e_{i}, \quad f_{i}^{*}=f_{i}, \quad\left(q^{h}\right)^{*}=q^{-h} .
$$

We denote by $U_{q}^{+}(\mathfrak{g})$ (resp. $\left.U_{q}^{-}(\mathfrak{g})\right)$ the subalgebra of $U_{q}(\mathfrak{g})$ generated by $e_{i}$ (resp. $f_{i}$ ) for $i \in I$. For $n \in \mathbb{Z}_{\geq 0}$ and $i \in I$, we set $e_{i}^{(n)}:=e_{i}^{n} /[n]_{i}$ ! and $f_{i}^{(n)}:=f_{i}^{n} /[n]_{i}$ !, where

$$
q_{i}=q^{\left(\alpha_{i}, \alpha_{i}\right) / 2}, \quad[n]_{i}=\frac{q_{i}^{n}-q_{i}^{-n}}{q_{i}-q_{i}^{-1}} \quad \text { and } \quad[n]_{i} !=\prod_{k=1}^{n}[k]_{i} .
$$

Let $U_{\mathbb{Z}\left[q, q^{-1}\right]}^{+}(\mathfrak{g})$ (resp. $\left.U_{\mathbb{Z}\left[q, q^{-1}\right]}^{-}(\mathfrak{g})\right)$ be the $\mathbb{Z}\left[q, q^{-1}\right]$-subalgebra of $U_{q}^{-}(\mathfrak{g})$ generated by $e_{i}^{(n)}$ (resp. $\left.f_{i}^{(n)}\right)$ for $i \in I$ and $n \in \mathbb{Z}_{\geq 0}$. The unipotent quantum coordinate ring is defined by

$$
A_{q}(\mathfrak{n})=\bigoplus_{\beta \in Q_{-}} A_{q}(\mathfrak{n})_{\beta}, \quad \text { where } A_{q}(\mathfrak{n})_{\beta}:=\operatorname{Hom}_{\mathbb{Q}(q)}\left(U_{q}^{+}(\mathfrak{g})_{-\beta}, \mathbb{Q}(q)\right),
$$

which is isomorphic to $U_{q}^{-}(\mathfrak{g})$ as a $\mathbb{Q}(q)$-algebra ([14, Lemma 8.2.2]). 
We define its $\mathbb{Z}\left[q, q^{-1}\right]$-form $A_{q}(\mathfrak{n})_{\mathbb{Z}\left[q, q^{-1}\right]}$ by

$$
A_{q}(\mathfrak{n})_{\mathbb{Z}\left[q, q^{-1}\right]}=\left\{a \in A_{q}(\mathfrak{n}) \mid\left\langle a, U_{\mathbb{Z}\left[q, q^{-1}\right]}^{+}(\mathfrak{g})\right\rangle \subset \mathbb{Z}\left[q, q^{-1}\right]\right\} .
$$

\subsection{Quiver Hecke algebras.}

Let $\mathbf{k}$ be a field. For $i, j \in I$, we choose polynomials $\mathcal{Q}_{i, j}(u, v) \in \mathbf{k}[u, v]$ such that

(a) $\mathcal{Q}_{i, j}(u, v)=\mathcal{Q}_{j, i}(v, u)$,

(b) it is of the form

$$
\mathcal{Q}_{i, j}(u, v)= \begin{cases}\sum_{p\left(\alpha_{i}, \alpha_{i}\right)+q\left(\alpha_{j}, \alpha_{j}\right)=-2\left(\alpha_{i}, \alpha_{j}\right)} t_{i, j ; p, q} u^{p} v^{q} & \text { if } i \neq j, \\ 0 & \text { if } i=j,\end{cases}
$$

where $t_{i, j ;-a_{i j}, 0} \in \mathbf{k}^{\times}$.

For $\beta \in \mathbf{Q}_{+}$with $\operatorname{ht}(\beta)=n$, we set

$$
I^{\beta}:=\left\{\nu=\left(\nu_{1}, \ldots, \nu_{n}\right) \in I^{n} \mid \sum_{k=1}^{n} \alpha_{\nu_{k}}=\beta\right\},
$$

on which the symmetric group $\mathfrak{S}_{n}=\left\langle s_{k} \mid k=1, \ldots, n-1\right\rangle$ acts by place permutations.

Definition 1.1. For $\beta \in \mathrm{Q}_{+}$, the quiver Hecke algebra $R(\beta)$ associated with $\mathrm{A}$ and $\left(\mathcal{Q}_{i, j}(u, v)\right)_{i, j \in I}$ is the $\mathbf{k}$-algebra generated by

$$
\left\{e(\nu) \mid \nu \in I^{\beta}\right\},\left\{x_{k} \mid 1 \leq k \leq n\right\},\left\{\tau_{l} \mid 1 \leq l \leq n-1\right\}
$$

satisfying the following defining relations:

$$
\begin{aligned}
& e(\nu) e\left(\nu^{\prime}\right)=\delta_{\nu, \nu^{\prime}} e(\nu), \sum_{\nu \in I^{\beta}} e(\nu)=1, x_{k} e(\nu)=e(\nu) x_{k}, x_{k} x_{l}=x_{l} x_{k}, \\
& \tau_{l} e(\nu)=e\left(s_{l}(\nu)\right) \tau_{l}, \tau_{k} \tau_{l}=\tau_{l} \tau_{k} \text { if }|k-l|>1, \\
& \tau_{k}^{2} e(\nu)=\mathcal{Q}_{\nu_{k}, \nu_{k+1}}\left(x_{k}, x_{k+1}\right) e(\nu), \\
& \left(\tau_{k} x_{l}-x_{s_{k}(l)} \tau_{k}\right) e(\nu)= \begin{cases}-e(\nu) & \text { if } l=k \text { and } \nu_{k}=\nu_{k+1}, \\
e(\nu) & \text { if } l=k+1 \text { and } \nu_{k}=\nu_{k+1}, \\
0 & \text { otherwise, }\end{cases} \\
& \left(\tau_{k+1} \tau_{k} \tau_{k+1}-\tau_{k} \tau_{k+1} \tau_{k}\right) e(\nu) \\
& = \begin{cases}\overline{\mathcal{Q}}_{\nu_{k}, \nu_{k+1}}\left(x_{k}, x_{k+1}, x_{k+2}\right) e(\nu) & \text { if } \nu_{k}=\nu_{k+2}, \\
0 & \text { otherwise },\end{cases}
\end{aligned}
$$


where

$$
\overline{\mathcal{Q}}_{i, j}(u, v, w)=\frac{\mathcal{Q}_{i, j}(u, v)-\mathcal{Q}_{i, j}(w, v)}{u-w} \in \mathbf{k}[u, v, w] .
$$

The algebra $R(\beta)$ has the $\mathbb{Z}$-grading defined by

$$
\operatorname{deg}(e(\nu))=0, \quad \operatorname{deg}\left(x_{k} e(\nu)\right)=\left(\alpha_{\nu_{k}}, \alpha_{\nu_{k}}\right), \quad \operatorname{deg}\left(\tau_{l} e(\nu)\right)=-\left(\alpha_{\nu_{l}}, \alpha_{\nu_{l+1}}\right) .
$$

Note that we have

$$
\tau_{k} a(x) e(\nu)=s_{k}(a(x)) \tau_{k} e(\nu)+\delta\left(\nu_{k}=\nu_{k+1}\right)\left(\partial_{k} a(x)\right) e(\nu)
$$

for $a(x) \in \mathbf{k}\left[x_{1}, \ldots, x_{n}\right]$. Here, $\delta(P)=1$ if $P$ is true and $\delta(P)=0$ otherwise, and $\partial_{k}$ is the difference operator

$$
\partial_{k} a(x)=\frac{s_{k} a(x)-a(x)}{x_{k}-x_{k+1}} .
$$

We denote by $R(\beta)$-Mod the category of graded $R(\beta)$-modules with degree preserving homomorphisms. We set $R(\beta)$-gmod to be the full subcategory of $R(\beta)$-Mod consisting of the modules which are finite-dimensional over $\mathbf{k}$, and set $R(\beta)$-proj to be the full subcategory of $R(\beta)$-Mod consisting of finitely generated projective graded $R(\beta)$-modules. We set $R$-Mod $:=\bigoplus_{\beta \in Q_{+}} R(\beta)$-Mod, $R$-proj $:=\bigoplus_{\beta \in Q_{+}} R(\beta)$-proj, and $R$-gmod $:=\bigoplus_{\beta \in Q_{+}} R(\beta)$-gmod. The trivial $R(0)$-module of degree 0 is denoted by 1 . For simplicity, we write "a module" instead of "a graded module". We define the grading shift functor $q$ by $(q M)_{k}=M_{k-1}$ for a graded module $M=\bigoplus_{k \in \mathbb{Z}} M_{k}$. For $M, N \in R(\beta)$-Mod, $\operatorname{Hom}_{R(\beta)}(M, N)$ denotes the space of degree preserving module homomorphisms. We define

$$
\operatorname{Hom}_{R(\beta)}(M, N):=\bigoplus_{k \in \mathbb{Z}} \operatorname{Hom}_{R(\beta)}\left(q^{k} M, N\right)
$$

and set $\operatorname{deg}(f):=k$ for $f \in \operatorname{Hom}_{R(\beta)}\left(q^{k} M, N\right)$. When $M=N$, we write $\operatorname{End}_{R(\beta)}(M)=$ $\operatorname{Hom}_{R(\beta)}(M, M)$. We sometimes write $R$ for $R(\beta)$ in $\operatorname{Hom}_{R(\beta)}(M, N)$ for simplicity.

For $M \in R(\beta)$-gmod, we set $M^{\star}:=\operatorname{HoM}_{\mathbf{k}}(M, \mathbf{k})$ with the $R(\beta)$-action given by

$$
(r \cdot f)(u):=f(\psi(r) u), \quad \text { for } f \in M^{\star}, r \in R(\beta) \text { and } u \in M,
$$

where $\psi$ is the antiautomorphism of $R(\beta)$ which fixes the generators. We say that $M$ is self-dual if $M \simeq M^{\star}$.

Let $e\left(\beta, \beta^{\prime}\right):=\sum_{\nu \in I^{\beta}, \nu^{\prime} \in I^{\beta^{\prime}}} e\left(\nu, \nu^{\prime}\right)$, where $e\left(\nu, \nu^{\prime}\right)$ is the idempotent corresponding to the concatenation $\nu * \nu^{\prime}$ of $\nu$ and $\nu^{\prime}$. Then there is an injective ring homomorphism

$$
R(\beta) \otimes R\left(\beta^{\prime}\right) \rightarrow e\left(\beta, \beta^{\prime}\right) R\left(\beta+\beta^{\prime}\right) e\left(\beta, \beta^{\prime}\right)
$$


given by $e(\nu) \otimes e\left(\nu^{\prime}\right) \mapsto e\left(\nu, \nu^{\prime}\right), x_{k} e(\beta) \otimes 1 \mapsto x_{k} e\left(\beta, \beta^{\prime}\right), 1 \otimes x_{k} e\left(\beta^{\prime}\right) \mapsto x_{k+\mathrm{ht}(\beta)} e\left(\beta, \beta^{\prime}\right)$, $\tau_{k} e(\beta) \otimes 1 \mapsto \tau_{k} e\left(\beta, \beta^{\prime}\right)$ and $1 \otimes \tau_{k} e\left(\beta^{\prime}\right) \mapsto \tau_{k+\mathrm{ht}(\beta)} e\left(\beta, \beta^{\prime}\right)$. For $a \in R(\beta)$ and $a^{\prime} \in R\left(\beta^{\prime}\right)$, the image of $a \otimes a^{\prime}$ is sometimes denoted by $a \otimes a^{\prime}$.

For $M \in R(\beta)$-Mod and $N \in R\left(\beta^{\prime}\right)$-Mod, we set

$$
M \circ N:=R\left(\beta+\beta^{\prime}\right) e\left(\beta, \beta^{\prime}\right) \otimes_{R(\beta) \otimes R\left(\beta^{\prime}\right)}(M \otimes N) .
$$

For $u \in M$ and $v \in N$, the image of $u \otimes v$ by the map $M \otimes N \rightarrow M \circ N$ is sometimes denoted by $u \otimes v$.

We denote by $M \nabla N$ the head of $M \circ N$ and by $M \Delta N$ the socle of $M \circ N$. We say that simple $R$-modules $M$ and $N$ strongly commute if $M \circ N$ is simple. A simple $R$-module $L$ is real if $L$ strongly commutes with itself. Note that if $M$ and $N$ strongly commute, then $M \circ N \simeq N \circ M$ up to a grading shift.

For $i \in I$ and the functors $E_{i}$ and $F_{i}$ are defined by

$$
E_{i}(M)=e\left(\alpha_{i}, \beta-\alpha_{i}\right) M \quad \text { and } \quad F_{i}(M)=R\left(\alpha_{i}\right) \circ M \quad \text { for an } R(\beta) \text {-module } M .
$$

For $i \in I$ and $n \in \mathbb{Z}_{>0}$, let $L(i)$ be the simple $R\left(\alpha_{i}\right)$-module concentrated on degree 0 and $P\left(i^{n}\right)$ the indecomposable projective $R\left(n \alpha_{i}\right)$-module whose head is isomorphic to $L\left(i^{n}\right):=q_{i}^{\frac{n(n-1)}{2}} L(i)^{\circ}$. For a simple module $M, \widetilde{F}_{i}(M)$ (resp. $\widetilde{E}_{i}(M)$ ) is the self-dual simple $R$-module being isomorphic to $L(i) \nabla M\left(\operatorname{resp} \operatorname{soc}\left(E_{i} M\right)\right)$. For $M \in R(\beta)$-Mod, we define

$$
F_{i}^{(n)} M:=P\left(i^{n}\right) \circ M, \quad E_{i}^{(n)} M:=\operatorname{Hom}_{R\left(n \alpha_{i}\right)}\left(P\left(i^{n}\right), e\left(n \alpha_{i}, \beta-n \alpha_{i}\right) M\right) .
$$

For $i \in I$ and $M \in R(\beta)$-Mod, we define

$$
\operatorname{wt}(M)=-\beta, \quad \varepsilon_{i}(M)=\max \left\{k \geq 0 \mid E_{i}^{k} M \neq 0\right\}, \quad \varphi_{i}(M)=\varepsilon_{i}(M)+\left\langle h_{i}, \operatorname{wt}(M)\right\rangle .
$$

We also can define $E_{i}^{*}, F_{i}^{*}, \varepsilon_{i}^{*}$, etc in the same manner as above if we replace the roles of $e\left(\alpha_{i}, \beta-\alpha_{i}\right)$ and $R\left(\alpha_{i}\right) \circ-$ with the ones of $e\left(\beta-\alpha_{i}, \alpha_{i}\right)$ and $-\circ R\left(\alpha_{i}\right)$.

We denote by $K(R$-proj) and $K(R$-gmod $)$ the Grothendieck groups of $R$-proj and $R$-gmod respectively.

Theorem 1.2 ([18, 19, 23]). There exist isomorphisms of $\mathbb{Z}\left[q, q^{-1}\right]$-bialgebras

$$
K(R \text {-proj }) \simeq U_{\mathbb{Z}\left[q, q^{-1}\right]}^{-}(\mathfrak{g}) \quad \text { and } \quad K(R \text {-gmod }) \simeq A_{q}(\mathfrak{n})_{\mathbb{Z}\left[q, q^{-1}\right]}
$$


For an $R(\beta)$-module $M$, we write

$$
\begin{aligned}
& e\left(i_{1}^{m_{1}}, \ldots, i_{t}^{m_{t}}, *\right) M:=e\left(m_{1} \alpha_{i_{1}}, \ldots, m_{t} \alpha_{i_{t}}, \beta-\sum_{k=1}^{t} m_{k} \alpha_{i_{k}}\right) M \\
& e\left(*, i_{1}^{m_{1}}, \ldots, i_{t}^{m_{t}}\right) M:=e\left(\beta-\sum_{k=1}^{t} m_{k} \alpha_{i_{k}}, m_{1} \alpha_{i_{1}}, \ldots, m_{t} \alpha_{i_{t}}\right) M .
\end{aligned}
$$

For a sequence $\left(i_{1}, \ldots, i_{m}\right)$ in $I$ such that $\left(\alpha_{i_{k}}, \alpha_{i_{k+1}}\right)<0(1 \leq k \leq m-1)$ and $\alpha_{i_{k}} \neq \alpha_{i_{k+2}}(1 \leq k \leq m-2)$, we denote by $L\left(i_{1}, \ldots, i_{m}\right)$ the one-dimensional $R(\beta)$ module $\left(\beta=\sum_{k=1}^{m} \alpha_{i_{k}}\right)$ with a basis $u$ on which $R(\beta)$ acts by $e\left(i_{1}, \ldots, i_{m}\right) u=u$, $x_{k} u=0, \tau_{k} u=0$.

\subsection{Cyclotomic quiver Hecke algebras.}

Let $A=\bigoplus_{n \in \mathbb{Z}} A_{n}$ be a commutative graded k-algebra such that $A_{n}=0$ for $n<0$. For $\beta \in \mathrm{Q}_{+}$, we define the graded algebra $R_{A}(\beta)$ by

$$
R_{A}(\beta):=A \otimes_{\mathbf{k}} R(\beta) .
$$

Let $\Lambda \in \mathrm{P}_{+}$and $t_{i}$ an indeterminate of degree $\left(\alpha_{i}, \alpha_{i}\right)$ for $i \in I$. A polynomial $f\left(t_{i}\right)=$ $\sum_{k=0}^{n} c_{k} t_{i}^{k} \in A\left[t_{i}\right]$ is homogeneous of degree $d$ if $c_{k} \in A_{d-\left(\alpha_{i}, \alpha_{i}\right) k}$. We say that $f\left(t_{i}\right)$ is quasi-monic if $c_{n} \in A_{0}^{\times}$. In this case, we write

$$
\operatorname{deg} f\left(t_{i}\right)=d \quad \text { and } \quad \operatorname{deg}_{t_{i}} f\left(t_{i}\right)=n .
$$

We choose a family of polynomials $a_{\Lambda}:=\left\{a_{\Lambda, i}\left(t_{i}\right)\right\}_{i \in I}$, where $a_{\Lambda, i}\left(t_{i}\right) \in A\left[t_{i}\right]$ is a quasimonic homogeneous polynomial with $\operatorname{deg}_{t_{i}} a_{\Lambda, i}\left(t_{i}\right)=\left\langle h_{i}, \Lambda\right\rangle$. Note that $\operatorname{deg}\left(a_{\Lambda, i}\left(t_{i}\right)\right)=$ $2\left(\alpha_{i}, \Lambda\right)$. Let $\lambda \in \Lambda-\mathrm{Q}_{+}$, and write $\beta:=\Lambda-\lambda$ and $n:=\operatorname{ht}(\beta)$. We define the cyclotomic quiver Hecke algebra

$$
R_{A}^{a_{\Lambda}}(\lambda):=\frac{R_{A}(\beta)}{\sum_{i \in I} R_{A}(\beta) a_{\Lambda, i}\left(x_{n} e\left(\beta-\alpha_{i}, \alpha_{i}\right)\right) R_{A}(\beta)} .
$$

The algebra $R_{A}^{a_{\Lambda}}(\lambda)$ is a finitely generated projective module over $A$ by $[11$, Remark 4.20].

Let $R_{A}^{a_{\Lambda}}(\lambda)$-Mod be the category of graded $R_{A}^{a_{\Lambda}}(\lambda)$-modules. We denote by $R_{A}^{a_{\Lambda}}(\lambda)$ -proj and $R_{A}^{a_{\Lambda}}(\lambda)$-gmod the category of finitely generated projective graded $R_{A}^{a_{\Lambda}}(\lambda)$ modules and the category of graded $R_{A}^{a_{\Lambda}}(\lambda)$-modules which are finite-dimensional over $\mathbf{k}$, respectively. Their morphisms are homogeneous homomorphisms of degree zero. Set

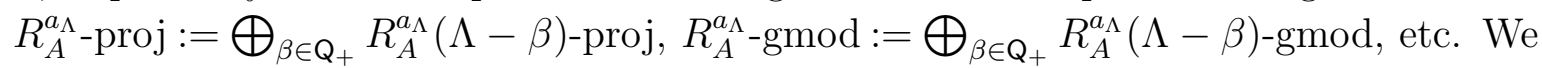


define the functors

$$
\begin{aligned}
& F_{i}^{a_{\Lambda}}: R_{A}^{a_{\Lambda}}(\lambda) \text {-Mod } \rightarrow R_{A}^{a_{\Lambda}}\left(\lambda-\alpha_{i}\right) \text {-Mod }, \\
& E_{i}^{a_{\Lambda}}: R_{A}^{a_{\Lambda}}(\lambda) \text {-Mod } \rightarrow R_{A}^{a_{\Lambda}}\left(\lambda+\alpha_{i}\right) \text {-Mod }
\end{aligned}
$$

by $F_{i}^{a_{\Lambda}} M=R_{A}^{a_{\Lambda}}\left(\lambda-\alpha_{i}\right) e\left(\alpha_{i}, \beta\right) \otimes_{R_{A}^{a_{\Lambda}}(\lambda)} M$ and $E_{i}^{a_{\Lambda}} M=e\left(\alpha_{i}, \beta-\alpha_{i}\right) M$ for $M \in$ $R_{A}^{a_{\Lambda}}(\lambda)$-Mod. They give a categorification of the simple $U_{q}(\mathfrak{g})$-module $V_{q}(\Lambda)$ with highest weight $\Lambda$.

When $A=\mathbf{k}$ and $a_{\Lambda, i}\left(t_{i}\right)=t_{i}^{\left\langle h_{i}, \Lambda\right\rangle}$ for $i \in I$, we simply write $R^{\Lambda}(\lambda), F_{i}^{\Lambda}, E_{i}^{\Lambda}$ instead of $R_{A}^{a_{\Lambda}}(\lambda), F_{i}^{a_{\Lambda}}, E_{i}^{a_{\Lambda}}$ respectively.

Theorem 1.3 ([11, Theorem 6.2]). For $\Lambda \in \mathrm{P}^{+}$, there exist $U_{\mathbb{Z}\left[q, q^{-1}\right]}(\mathfrak{g})$-module isomorphisms

$$
K\left(R^{\Lambda} \text {-proj }\right) \simeq V_{\mathbb{Z}\left[q, q^{-1}\right]}(\Lambda), \quad K\left(R^{\Lambda} \text {-gmod }\right) \simeq V_{\mathbb{Z}\left[q, q^{-1}\right]}(\Lambda)^{\vee}
$$

For $n \in \mathbb{Z}_{\geq 0}$, we define

$$
\begin{aligned}
& F_{i}^{a_{\Lambda}(n)} M=\operatorname{Hom}_{R_{A}\left(n \alpha_{i}\right)}\left(P_{A}\left(i^{n}\right),\left(F_{i}^{a_{\Lambda}}\right)^{n} M\right), \\
& E_{i}^{a_{\Lambda}(n)} M=\operatorname{Hom}_{R_{A}\left(n \alpha_{i}\right)}\left(P_{A}\left(i^{n}\right),\left(E_{i}^{a_{\Lambda}}\right)^{n} M\right),
\end{aligned}
$$

which give exact functors:

$$
\begin{aligned}
& F_{i}^{a_{\Lambda}(n)}: R_{A}^{a_{\Lambda}}(\lambda)-\operatorname{Mod} \rightarrow R_{A}^{a_{\Lambda}}\left(\lambda-n \alpha_{i}\right) \text {-Mod }, \\
& E_{i}^{a_{\Lambda}(n)}: R_{A}^{a_{\Lambda}}(\lambda)-\operatorname{Mod} \rightarrow R_{A}^{a_{\Lambda}}\left(\lambda+n \alpha_{i}\right) \text {-Mod } .
\end{aligned}
$$

Then the following lemma is an easy consequence of the theory of $\mathfrak{s l}_{2}$-categorification due to Rouquier ([22]).

Proposition 1.4. Let $\Lambda \in \mathrm{P}^{+}$and $\lambda \in \mathrm{W} \Lambda$ such that $n:=\left\langle h_{i}, \lambda\right\rangle \geq 0$. Then we have category equivalences, quasi-inverse to each other:

$$
R_{A}^{a_{\Lambda}}(\lambda)-\operatorname{Mod} \underset{E_{i}^{a_{\Lambda}(n)}}{\stackrel{F_{i}^{a_{\Lambda}(n)}}{\rightleftarrows}} R_{A}^{a_{\Lambda}}\left(s_{i} \lambda\right)-\operatorname{Mod} .
$$

In particular, we have $Z\left(R_{A}^{a_{\Lambda}}(\lambda)\right) \simeq Z\left(R_{A}^{a_{\Lambda}}\left(s_{i} \lambda\right)\right)$. Here $Z(R)$ denotes the center of an algebra $R$.

Note that the last assertion follows from $Z(R) \simeq \operatorname{End}\left(\operatorname{id}_{R \text {-Mod }}\right)$. 


\subsection{The categories $\mathscr{C}_{w}$.}

In this subsection, we review briefly convex orders and the categories $\mathscr{C}_{w}[15,24]$.

\section{Definition 1.5.}

(i) A preorder $\preceq$ on a set $X$ is a binary relation on $X$ satisfying

(a) $x \preceq x$ for any $x \in X$,

(b) if $x \preceq y$ and $y \preceq z$ for $x, y, z \in X$, then $x \preceq z$.

(ii) We say that a preorder $\preceq$ on $X$ is total if we have either $x \preceq y$ or $y \preceq x$ for any $x, y \in X$.

(iii) For a preorder $\preceq$, we say that $x$ and $y$ are $\preceq$-equivalent if $x \preceq y$ and $y \preceq x$. The equivalence class for $\preceq$ is called $\preceq$-equivalence class.

(iv) For subsets $A$ and $B$, we write $A \preceq B$ if $a \preceq b$ for any $a \in A$ and $b \in B$.

Definition 1.6. A face is a decomposition of a subset $X$ of an $\mathbb{R}$-vector space into three disjoint subsets $X=A_{-} \sqcup A_{0} \sqcup A_{+}$such that

$$
\begin{aligned}
& \left(\operatorname{span}_{\mathbb{R}_{\geq 0}} A_{+}+\operatorname{span}_{\mathbb{R}} A_{0}\right) \cap \operatorname{span}_{\mathbb{R}_{\geq 0}} A_{-}=\{0\}, \\
& \left(\operatorname{span}_{\mathbb{R}_{\geq 0}} A_{-}+\operatorname{span}_{\mathbb{R}} A_{0}\right) \cap \operatorname{span}_{\mathbb{R}_{\geq 0}} A_{+}=\{0\},
\end{aligned}
$$

where $\operatorname{span}_{\mathbb{R}} S$ is the $\mathbb{R}$-vector space spanned by $S$ and $\operatorname{span}_{\mathbb{R}_{\geq 0}} S$ is the subset of $\operatorname{span}_{\mathbb{R}} S$ whose elements are linear combinations of $S$ with non-negative coefficients.

We set $\operatorname{span}_{\mathbb{R}} \emptyset=\operatorname{span}_{\mathbb{R}_{\geq 0}} \emptyset=\{0\}$.

Definition 1.7. Let $V$ be an $\mathbb{R}$-vector space and let $X$ be a subset of $V \backslash\{0\}$.

(i) A convex preorder $\preceq$ on $X$ is a total preorder on $X$ such that, for any $\preceq$ equivalence class $\mathcal{C}$, the triple $(\{x \in X \mid x \prec \mathcal{C}\}, \mathcal{C},\{x \in X \mid x \succ \mathcal{C}\})$ is a face.

(ii) A convex preorder $\preceq$ on $X$ is called a convex order if every $\preceq$-equivalence class is of the form $X \cap l$ for some line $l$ in $V$ through the origin.

For each reduced expression of $w \in \mathbf{W}$, one can define a corresponding convex order on $\Delta_{+}$as follows.

Proposition 1.8 ([15, Proposition 1.24]). Let $\underline{w}=s_{i_{1}} s_{i_{2}} \cdots s_{i_{l}}$ be a reduced expression of $w \in \mathrm{W}$. We set $\beta_{k}:=s_{i_{1}} \cdots s_{i_{k-1}}\left(\alpha_{i_{k}}\right)$ so that $\Delta_{+} \cap w \Delta_{-}=\left\{\beta_{1}, \ldots, \beta_{l}\right\}$. Then there is a convex order $\preceq$ on $\Delta_{+}$such that

$$
\beta_{1} \prec \beta_{2} \prec \cdots \prec \beta_{l} \prec \gamma
$$

for any $\gamma \in \Delta_{+} \cap w \Delta_{+}$. 
We fix a convex order $\preceq$ on $\Delta_{+}$given in Proposition 1.8. Note that it can be extended uniquely to the convex order on $\mathbb{Z}_{>0} \Delta_{+}:=\left\{n \gamma \mid n \in \mathbb{Z}_{>0}, \gamma \in \Delta_{+}\right\}$, which means that we write $n \beta \preceq n^{\prime} \beta^{\prime}$ for $\beta, \beta^{\prime} \in \Delta_{+}$if $\beta \preceq \beta^{\prime}$.

For $M \in R(\beta)$-Mod, we define

$$
\begin{aligned}
\mathrm{W}(M) & :=\left\{\gamma \in \mathrm{Q}_{+} \cap\left(\beta-\mathrm{Q}_{+}\right) \mid e(\gamma, \beta-\gamma) M \neq 0\right\}, \\
\mathrm{W}^{*}(M) & :=\left\{\gamma \in \mathrm{Q}_{+} \cap\left(\beta-\mathrm{Q}_{+}\right) \mid e(\beta-\gamma, \gamma) M \neq 0\right\} .
\end{aligned}
$$

Let $L$ be a simple $R(\beta)$-module for $\beta \in \mathrm{Q}_{+}$. We say that $L$ is $\preceq$-cuspidal if

(a) $\beta \in \mathbb{Z}_{>0} \Delta_{+}$,

(b) $\mathbf{W}(L) \subset \operatorname{span}_{\mathbb{R}_{\geq 0}}\left\{\gamma \in \Delta_{+} \mid \gamma \preceq \beta\right\}$.

Theorem 1.9 ([15, Theorem 2.8]). For a simple $R$-module L, there exists a unique sequence $\left(L_{1}, L_{2}, \ldots, L_{h}\right)$ of $\preceq$-cuspidal modules (up to isomorphisms) such that

(i) $-\mathrm{wt}\left(L_{k}\right) \succ-\mathrm{wt}\left(L_{k+1}\right)$ for $k=1, \ldots, h-1$,

(ii) $L$ is isomorphic to the head of $L_{1} \circ L_{2} \circ \cdots \circ L_{h}$.

We set $\mathfrak{d}_{\preceq}(L):=\left(L_{1}, \ldots, L_{h}\right)$ in Theorem 1.9 , which is called the $\preceq$-cuspidal decomposition of $L$. We write $\mathfrak{d}$ instead of $\mathfrak{d} \preceq$ for simplicity when there is no afraid of confusion.

For $w \in \mathrm{W}$, we denote by $\mathscr{C}_{w}$ the full subcategory of $R$-gmod whose objects $M$ satisfy

$$
\mathrm{W}(M) \subset \operatorname{span}_{\mathbb{R}_{\geq 0}}\left(\Delta_{+} \cap w \Delta_{-}\right) .
$$

Proposition 1.10 ([15, Proposition 2.18]). Let $\underline{w}=s_{i_{1}} s_{i_{2}} \cdots s_{i_{\ell}}$ be a reduced expression of $w \in \mathrm{W}$, and $\beta_{\ell}=s_{i_{1}} \cdots s_{i_{\ell-1}}\left(\alpha_{i_{\ell}}\right)$. We take a simple $R$-module $L$ and set

$$
\mathfrak{d}(L):=\left(L_{1}, L_{2}, \ldots, L_{h}\right), \quad \gamma_{k}:=-\mathrm{wt}\left(L_{k}\right) \quad \text { for } k=1, \ldots, h \text {. }
$$

Then we have that $L \in \mathscr{C}_{w}$ if and only if $\beta_{\ell} \succeq \gamma_{1}$.

By the construction and Proposition $1.10, \mathscr{C}_{w}$ is the smallest monoidal abelian full subcategory of $R$-gmod such that

(a) $\mathscr{C}_{w}$ is stable under the subquotients, extension, and grading shifts,

(b) $\mathscr{C}_{w}$ contains all the $\preceq$-cuspidal modules corresponding to $\beta_{k}=s_{i_{1}} \cdots s_{i_{k-1}}\left(\alpha_{i_{k}}\right)$ for $k=1, \ldots, \ell$.

It was proved in [14] that $\mathscr{C}_{w}$ gives a monoidal categorification of the quantum unipotent coordinate algebra $A_{q}\left(\mathfrak{n}\left(w^{-1}\right)\right)$ when it is of symmetric type. 
1.5. Monoidal categories. We shall review monoidal categories and related notions. We refer the reader to [12, Appendix A] and [17] for more details.

A monoidal category (or tensor category) is a datum consisting of

(a) a category $\mathcal{T}$

(b) a bifunctor $\otimes \cdot: \mathcal{T} \times \mathcal{T} \rightarrow \mathcal{T}$,

(c) an isomorphism $a(X, Y, Z):(X \otimes Y) \otimes Z \stackrel{\sim}{\longrightarrow} X \otimes(Y \otimes Z)$ which is functorial in $X, Y, Z \in \mathcal{T}$,

(d) an object $\mathbf{1}$ endowed with an isomorphism $\epsilon: \mathbf{1} \otimes \mathbf{1} \stackrel{\sim}{\longrightarrow}$

such that

(1) the diagram below commutes for all $X, Y, Z, W \in \mathcal{T}$ :

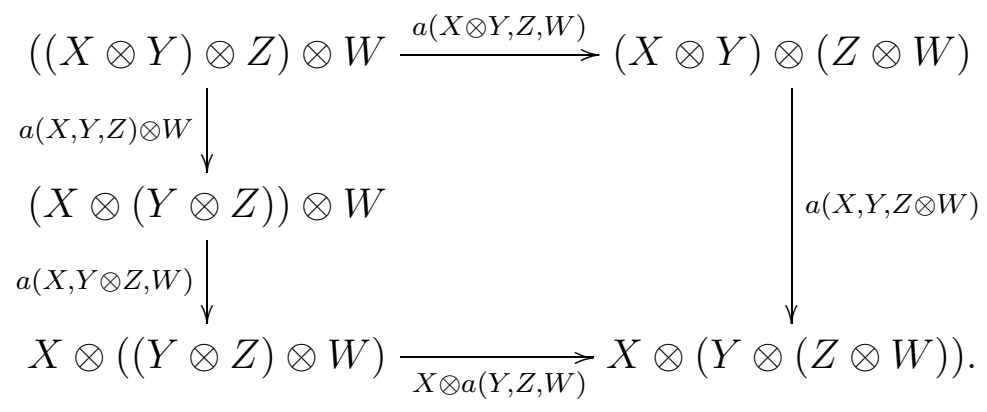

(2) the functors $\mathcal{T} \ni X \mapsto \mathbf{1} \otimes X \in \mathcal{T}$ and $\mathcal{T} \ni X \mapsto X \otimes \mathbf{1} \in \mathcal{T}$ are fully faithful.

We call $\mathbf{1}$ a unit object of $\mathcal{T}$. We have canonical isomorphisms $\mathbf{1} \otimes X \simeq X \otimes \mathbf{1} \simeq X$ for any $X \in \mathcal{T}$. For $n \in \mathbb{Z}_{>0}$ and $X \in \mathcal{T}$, we set $X^{\otimes n}=\underbrace{X \otimes \cdots \otimes X}_{n \text { times }}$, and $X^{\otimes 0}=1$.

For monoidal categories $\mathcal{T}$ and $\mathcal{T}^{\prime}$, a functor $F: \mathcal{T} \rightarrow \mathcal{T}^{\prime}$ is called a monoidal functor if it is endowed with an isomorphism $\xi_{F}: F(X \otimes Y) \stackrel{\sim}{\longrightarrow} F(X) \otimes F(Y)$ which is functorial in $X, Y \in \mathcal{T}$ such that the diagram

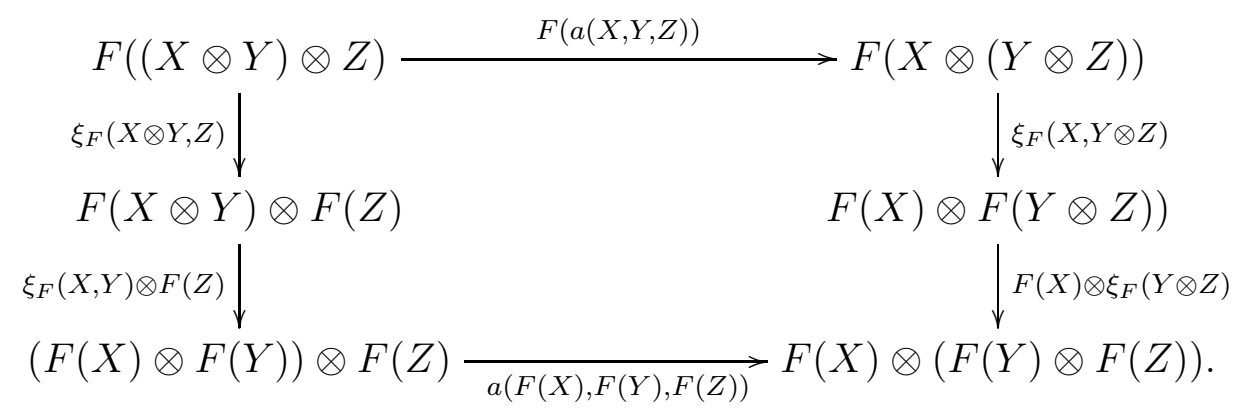

commutes for all $X, Y, Z \in \mathcal{T}$. We omit to write $\xi_{F}$ for simplicity. A monoidal functor $F$ is called unital if $(F(\mathbf{1}), F(\epsilon))$ is a unit object. In this paper, we simply write a "monoidal functor" for a unital monoidal functor. 
We say that a monoidal category $\mathcal{T}$ is an additive (resp. abelian) monoidal category if $\mathcal{T}$ is additive (resp. abelian) and the bifunctor $\cdot \otimes \cdot$ is bi-additive. Similarly, for a commutative ring $\mathbf{k}$, a monoidal category $\mathcal{T}$ is $\mathbf{k}$-linear if $\mathcal{T}$ is $\mathbf{k}$-linear and the bifunctor - $\otimes \cdot$ is $\mathbf{k}$-bilinear.

An object $X \in \mathcal{T}$ is invertible if the functors $\mathcal{T} \rightarrow \mathcal{T}$ given by $Z \mapsto Z \otimes X$ and $Z \mapsto X \otimes Z$ are equivalence of categories. If $X$ is invertible, then one can find an object $Y$ and isomorphisms $f: X \otimes Y \stackrel{\sim}{\rightarrow} \mathbf{1}$ and $g: Y \otimes X \stackrel{\sim}{\rightarrow} \mathbf{1}$ such that the diagrams below commute:

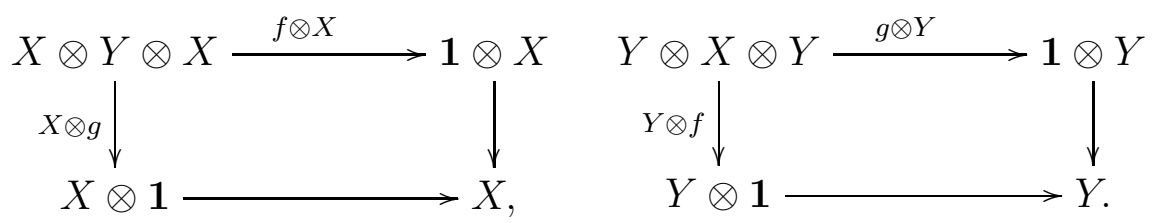

The triple $(Y, f, g)$ is unique up to a unique isomorphism. We write $Y=X^{\otimes-1}$.

Adjunctions.

Definition 1.11. Let $X$ and $Y$ be objects in a monoidal category $\mathcal{T}$, and $\varepsilon: X \otimes Y \rightarrow \mathbf{1}$ and $\eta: \mathbf{1} \rightarrow Y \otimes X$ morphisms in $\mathcal{T}$.

(i) We say that $(\varepsilon, \eta)$ is an adjunction if the following are satisfied:

(a) the composition $X \simeq X \otimes \mathbf{1} \stackrel{X \otimes \eta}{\longrightarrow} X \otimes Y \otimes X \stackrel{\varepsilon \otimes X}{\longrightarrow} \mathbf{1} \otimes X \simeq X$ is the identity of $X$,

(b) the composition $Y \simeq \mathbf{1} \otimes Y \stackrel{\eta \otimes Y}{\longrightarrow} Y \otimes X \otimes Y \stackrel{Y \otimes \varepsilon}{\longrightarrow} Y \otimes \mathbf{1} \simeq Y$ is the identity of $Y$.

(ii) The pair $(\varepsilon, \eta)$ is a quasi-adjunction if the compositions $X \otimes \mathbf{1} \stackrel{X \otimes \eta}{\longrightarrow} X \otimes Y \otimes$ $X \stackrel{\varepsilon \otimes X}{\longrightarrow} \mathbf{1} \otimes X$ and $\mathbf{1} \otimes Y \stackrel{\eta \otimes Y}{\longrightarrow} Y \otimes X \otimes Y \stackrel{Y \otimes \varepsilon}{\longrightarrow} Y \otimes \mathbf{1}$ are isomorphisms.

In the case when $(\varepsilon, \eta)$ is an adjunction, we say that the pair $(X, Y)$ is called a dual pair, or $X$ is a left dual to $Y$ and $Y$ is a right dual to $X$. Note that a left dual (resp. right dual) of an object is unique up to a unique isomorphism if it exists.

Definition 1.12. A monoidal category $\mathcal{T}$ is rigid if every object in $\mathcal{T}$ has left and right duals.

Lemma 1.13. Let $(X, Y)$ be a dual pair in a monoidal category $\mathcal{T}$. Then for $Z, W \in \mathcal{T}$, there are isomorphisms

$$
\operatorname{Hom}_{\mathcal{T}}(Z, W \otimes X) \simeq \operatorname{Hom}_{\mathcal{T}}(Z \otimes Y, W), \quad \operatorname{Hom}_{\mathcal{T}}(X \otimes Z, W) \simeq \operatorname{Hom}_{\mathcal{T}}(Z, Y \otimes W) .
$$

Lemma 1.14 ([12, Lemma A.2]). Let $\mathcal{T}$ be a monoidal category and $\varepsilon: X \otimes Y \rightarrow \mathbf{1}$ be a morphism in $\mathcal{T}$. Then the following are equivalent. 
(i) There exists a morphism $\eta: \mathbf{1} \rightarrow Y \otimes X$ such that $(\varepsilon, \eta)$ is an adjunction.

(ii) There exists a morphism $\eta: \mathbf{1} \rightarrow Y \otimes X$ such that $(\varepsilon, \eta)$ is a quasi-adjunction.

(iii) the map $\operatorname{Hom}_{\mathcal{T}}(Z, W \otimes X) \rightarrow \operatorname{Hom}_{\mathcal{T}}(Z \otimes Y, W)$ that associates $f: Z \rightarrow W \otimes X$ the morphism $Z \otimes Y \stackrel{f \otimes Y}{\longrightarrow} W \otimes X \otimes Y \stackrel{W \otimes \varepsilon}{\longrightarrow} W$ is bijective for any $Z, W \in \mathcal{T}$.

(iv) the map $\operatorname{Hom}_{\mathcal{T}}(Z, Y \otimes W) \rightarrow \operatorname{Hom}_{\mathcal{T}}(X \otimes Z, W)$ that associates $f: Z \rightarrow Y \otimes W$ the morphism $X \otimes Z \stackrel{X \otimes f}{\longrightarrow} X \otimes Y \otimes W \stackrel{\varepsilon \otimes W}{\longrightarrow} W$ is bijective for any $Z, W \in \mathcal{T}$.

Moreover if these equivalent conditions are satisfied, the morphism $\eta$ in (i) is unique.

Lemma 1.15 ([12, Lemma A.3]). Let $\mathcal{T}$ be a $\mathbf{k}$-linear monoidal category such that $\cdot \otimes$. is exact. Let

$$
0 \rightarrow X^{\prime} \stackrel{f}{\longrightarrow} X \stackrel{f^{\prime}}{\longrightarrow} X^{\prime \prime} \rightarrow 0 \quad \text { and } \quad 0 \rightarrow Y^{\prime \prime} \stackrel{g^{\prime}}{\longrightarrow} Y \stackrel{g}{\longrightarrow} Y^{\prime} \longrightarrow 0
$$

be exact sequences and let

$$
\begin{aligned}
& \varepsilon^{\prime}: X^{\prime} \otimes Y^{\prime} \rightarrow \mathbf{1}, \quad \varepsilon: X \otimes Y \rightarrow \mathbf{1}, \quad \varepsilon^{\prime \prime}: X^{\prime \prime} \otimes Y^{\prime \prime} \rightarrow \mathbf{1}, \\
& \eta^{\prime}: \mathbf{1} \rightarrow Y^{\prime} \otimes X^{\prime}, \quad \eta: \mathbf{1} \rightarrow Y \otimes X, \quad \eta^{\prime \prime}: \mathbf{1} \rightarrow Y^{\prime \prime} \otimes X^{\prime \prime}
\end{aligned}
$$

be morphisms such that the diagrams below commute up to constant multiples:
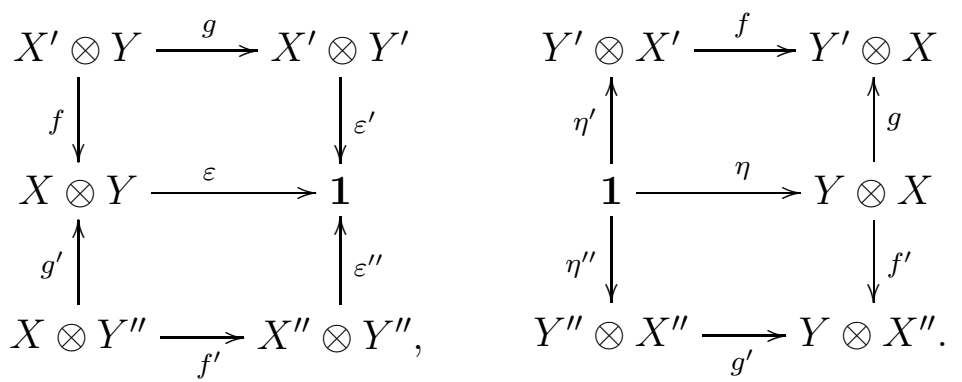

If $\left(\varepsilon^{\prime}, \eta^{\prime}\right)$ and $\left(\varepsilon^{\prime \prime}, \eta^{\prime \prime}\right)$ are quasi-adjunctions, then $(\varepsilon, \eta)$ is also a quasi-adjunction.

Commuting families. We now consider a family $\left\{P_{i}\right\}_{i \in I}$ of objects and a family of isomorphisms $\left\{B_{i, j}: P_{i} \otimes P_{j} \stackrel{\sim}{\longrightarrow} P_{j} \otimes P_{i}\right\}_{i, j \in I}$ in a monoidal category $\mathcal{T}$. We say that $\left(\left\{P_{i}\right\}_{i \in I},\left\{B_{i, j}\right\}_{i, j \in I}\right)$ is a commuting family if it satisfies the following:

(a) $B_{i, i}=\mathrm{id}_{P_{i} \otimes P_{i}}$ for any $i \in I$,

(b) $B_{j, i} \circ B_{i, j}=\mathrm{id}_{P_{i} \otimes P_{j}}$ for any $i, j \in I$, 
(c) the isomorphisms $B_{i, j}$ satisfy the Yang-Baxter equation, which means that the following diagram commutes for any $i, j, k \in I$ :

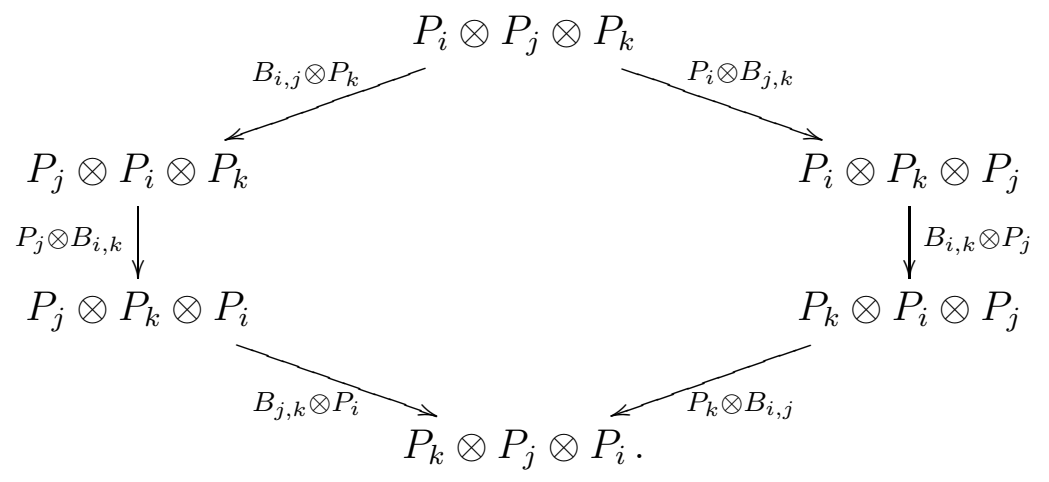

We set

$$
\Gamma:=\mathbb{Z}^{\oplus I} \quad \text { and } \quad \Gamma_{\geq 0}:=\mathbb{Z}_{\geq 0}^{\oplus I} .
$$

For $i \in I$, let $e_{i}$ be the image of 1 under the canonical embedding $\mathbb{Z} \longmapsto \Gamma$ to the $i$-th component so that $\left\{e_{i} \mid i \in I\right\}$ is a basis of $\Gamma$. Then we have the following lemma.

Lemma 1.16 ([12, Section A.4]). Let $\left(\left\{P_{i}\right\}_{i \in I},\left\{B_{i, j}\right\}_{i, j \in I}\right)$ is a commuting family in a monoidal category $\mathcal{T}$. Then there exist

(a) an object $P^{\alpha}$ for any $\alpha \in \Gamma_{\geq 0}$,

(b) an isomorphism $\xi_{\alpha, \beta}: P^{\alpha} \otimes P^{\beta} \stackrel{\sim}{\longrightarrow} P^{\alpha+\beta}$ for any $\alpha, \beta \in \Gamma_{\geq 0}$

such that

(i) $P^{0}=1$ and $P^{e_{i}}=P_{i}$ for $i \in I$,

(ii) the diagram

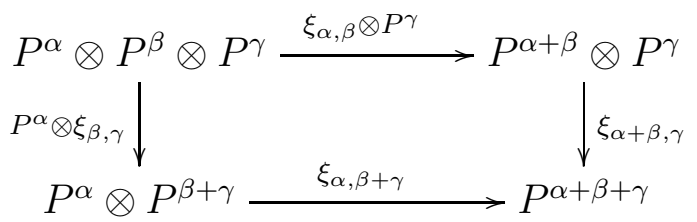

commutes for any $\alpha, \beta, \gamma \in \Gamma_{\geq 0}$,

(iii) the diagrams

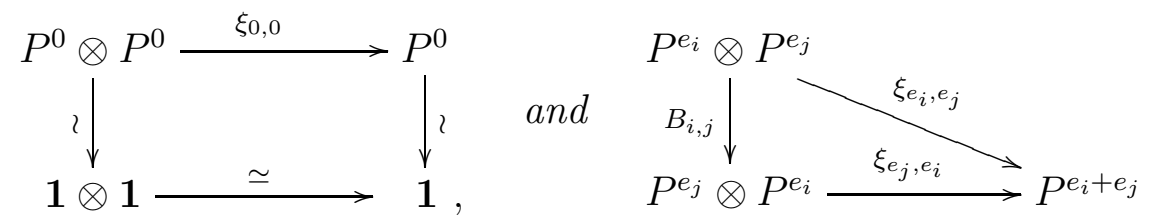

commute for any $i, j \in I$. 
Moreover, such a datum $\left(\left\{P^{\alpha}\right\}_{\alpha \in \Gamma_{\geq 0}},\left\{\xi_{\alpha, \beta}\right\}_{\alpha, \beta \in \Gamma_{\geq 0}}\right)$ is unique up to a unique isomorphism.

\section{LOCALIZATIONS}

A localization of a monoidal category using a commuting family of central objects was explained in [12, Appendix A]. In this section, we shall generalize this localization to a real commuting family of (graded) braiders.

\subsection{Real commuting family of braiders.}

\section{Definition 2.1.}

(i) A left braider of a monoidal category $\mathcal{T}$ is a pair $\left(C, R_{C}\right)$ of an object $C$ and a morphism

$$
R_{C}(X): C \otimes X \longrightarrow X \otimes C
$$

which is functorial in $X \in \mathcal{T}$ such that the following diagrams commutes:
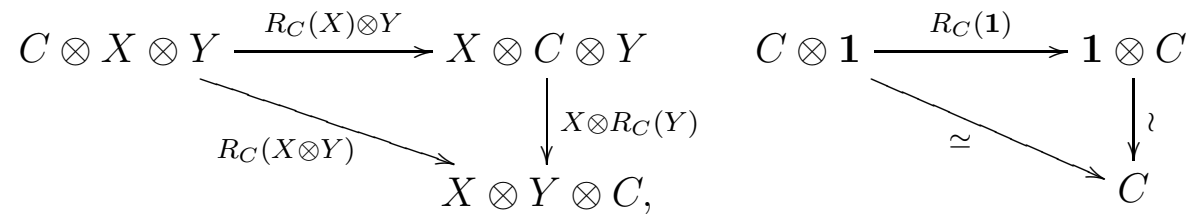

(ii) Assume that $\mathcal{T}$ is a $\mathbf{k}$-linear monoidal category. A left braider $\left(C, R_{C}\right)$ is called real if $R_{C}(C) \in \mathbf{k}^{\times} \mathrm{id}_{C \otimes C}$.

Similarly we can define the notion of right braiders by reversing the order of tensor products. Since we treat mainly left braiders in this paper, we simply say braiders for left braiders in the sequel.

Note that, for $f \in \operatorname{Hom}_{\mathcal{T}}(X, Y)$, the diagram

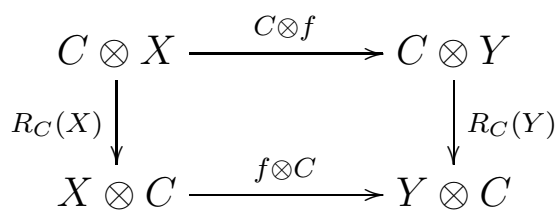

commutes since $R_{C}$ is a natural transformation between left and right tensoring with $C$ by the definition.

A braider $\left(C, R_{C}\right)$ is called a central object if $R_{C}(X)$ is an isomorphism for any $X \in \mathcal{T}$. 
Let $\mathcal{T}_{\mathrm{br}}$ be the category of braiders in $\mathcal{T}$. A morphism from $\left(C, R_{C}\right)$ to $\left(C^{\prime}, R_{C^{\prime}}\right)$ in $\mathcal{T}_{\text {br }}$ is a morphism $f \in \operatorname{Hom}_{\mathcal{T}}\left(C, C^{\prime}\right)$ such that the following diagram commutes for any $X \in \mathcal{T}:$

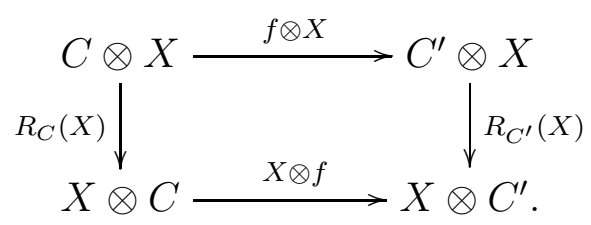

For braiders $\left(C_{1}, R_{C_{1}}\right)$ and $\left(C_{2}, R_{C_{2}}\right)$ of $\mathcal{T}$, let $R_{C_{1} \otimes C_{2}}(X)$ be the composition

$$
C_{1} \otimes C_{2} \otimes X \stackrel{R_{C_{2}}(X)}{\longrightarrow} C_{1} \otimes X \otimes C_{2} \stackrel{R_{C_{1}}(X)}{\longrightarrow} X \otimes C_{1} \otimes C_{2}
$$

for $X \in \mathcal{T}$. Then, one can show that $\left(C_{1} \otimes C_{2}, R_{C_{1} \otimes C_{2}}\right)$ is also a braider of $\mathcal{T}$. Thus the category $\mathcal{T}_{\text {br }}$ has a structure of a monoidal category, and there is a canonical faithful monoidal functor $\mathcal{T}_{\text {br }} \rightarrow \mathcal{T}$. We regard an object of $\mathcal{T}_{\text {br }}$ as an object of $\mathcal{T}$ if there is no afraid of confusion.

The morphism $R_{C_{1}}\left(C_{2}\right): C_{1} \otimes C_{2} \rightarrow C_{2} \otimes C_{1}$ is a morphism of braiders because the following diagram commutes:

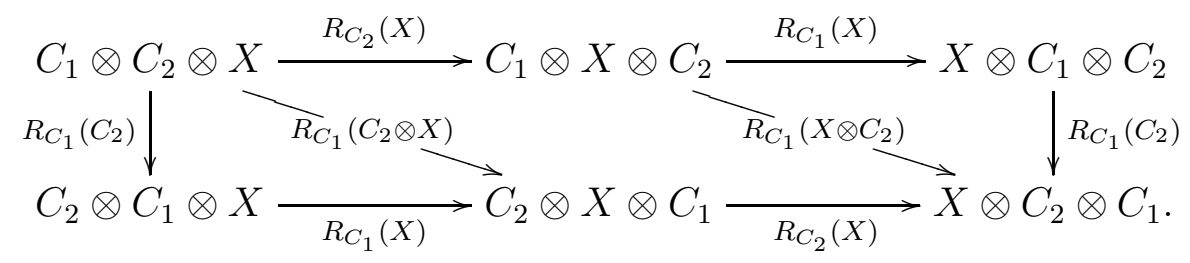

Let $\mathbf{k}$ be a commutative ring with unity, and let $\mathcal{T}$ be a $\mathbf{k}$-linear monoidal category.

Definition 2.2 (cf. Example 4.7). Let $I$ be an index set and let $\left\{\left(C_{i}, R_{C_{i}}\right)\right\}_{i \in I}$ be a family of braiders in $\mathcal{T}$. We say that $\left(C_{i}, R_{C_{i}}\right)_{i \in I}$ is a real commuting family of braiders if

(a) $R_{C_{i}}\left(C_{i}\right) \in \mathbf{k}^{\times} \mathrm{id}_{C_{i} \otimes C_{i}}$,

(b) $R_{C_{j}}\left(C_{i}\right) \circ R_{C_{i}}\left(C_{j}\right) \in \mathbf{k}^{\times} \mathrm{id}_{C_{i} \otimes C_{j}}$.

Note that the morphisms $R_{C_{j}}\left(C_{i}\right)$ satisfy the Yang-Baxter equation (1.4).

Lemma 2.3. Let $\left(C_{i}, R_{C_{i}}\right)_{i \in I}$ be a real commuting family of braiders in $\mathcal{T}$.

(i) Then there exists a family $\left\{\eta_{i j}\right\}_{i, j \in I}$ of elements in $\mathbf{k}^{\times}$such that

$$
\begin{aligned}
R_{C_{i}}\left(C_{i}\right) & =\eta_{i i} \text { id }_{C_{i} \otimes C_{i}}, \\
R_{C_{j}}\left(C_{i}\right) \circ R_{C_{i}}\left(C_{j}\right) & =\eta_{i j} \eta_{j i} \text { id }_{C_{i} \otimes C_{j}}
\end{aligned}
$$

for all $i, j \in I$. 
(ii) Let $\left\{\eta_{i j}\right\}_{i, j \in I}$ be as in (i) and set $B_{i j}=\eta_{i j}^{-1} R_{C_{i}}\left(C_{j}\right)$. Then $\left(\left\{C_{i}\right\}_{i \in I},\left\{B_{i j}\right\}_{i, j \in I}\right)$ is a commuting family in the monoidal category $\mathcal{T}_{\mathrm{br}}$.

Proof. (i) Write

$$
\begin{array}{r}
R_{C_{i}}\left(C_{i}\right)=c_{i} \text { id }_{C_{i} \otimes C_{i}}, \\
R_{C_{j}}\left(C_{i}\right) \circ R_{C_{i}}\left(C_{j}\right)=c_{i j} \operatorname{id}_{C_{i} \otimes C_{j}}
\end{array}
$$

with $c_{i}, c_{i j} \in \mathbf{k}^{\times}$. Then we can check easily that $c_{i j} \operatorname{id}_{C_{i} \otimes C_{j}}=c_{j i} \operatorname{id}_{C_{i} \otimes C_{j}}$. Taking a total order $\prec$ on $I$, we obtain the assertion by setting

$$
\eta_{i j}= \begin{cases}c_{i} & \text { if } i=j, \\ c_{i j} & \text { if } i \prec j, \\ 1 & \text { if } j \prec i .\end{cases}
$$

(ii) is obvious.

Let $\left(C_{i}, R_{C_{i}}\right)_{i \in I}$ be a real commuting family of braiders in $\mathcal{T}$. Thanks to Lemma 1.16 and Lemma 2.3, we can find

(a) a braider $\left(C^{\alpha}, R_{C^{\alpha}}\right)$ for each $\alpha \in \Gamma_{\geq 0}$,

(b) an isomorphism $\xi_{\alpha, \beta}: C^{\alpha} \otimes C^{\beta} \stackrel{\sim}{\longrightarrow} C^{\alpha+\beta}$ for $\alpha, \beta \in \Gamma_{\geq 0}$

satisfying the conditions (i), (ii) and (iii) of Lemma 1.16. Here the right diagram in (1.6) reads as: for $i, j \in I$,

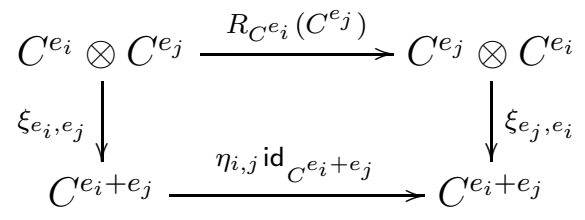

commutes for $\eta_{i, j} \in \mathbf{k}^{\times}$as in Lemma 2.3 (i). Note that $\xi_{\alpha, \beta}$ is a morphism of braiders, i.e. the diagram

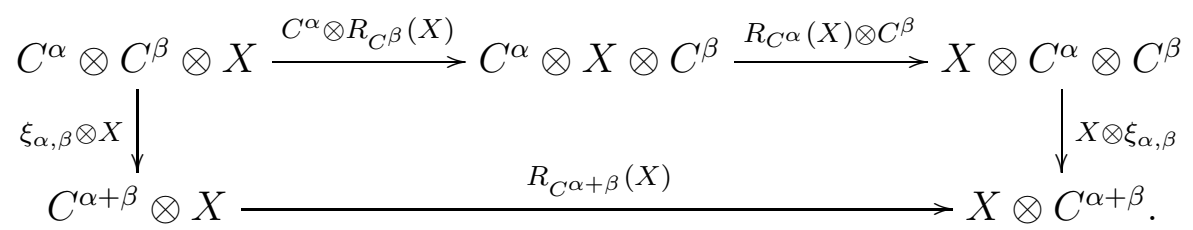

commutes for $X \in \mathcal{T}$.

We define

$$
\eta(\alpha, \beta):=\prod_{i, j \in I} \eta_{i, j}^{a_{i} b_{j}} \in \mathbf{k}^{\times}
$$


for $\alpha=\sum_{i \in I} a_{i} e_{i}$ and $\beta=\sum_{j \in I} b_{j} e_{j}$ in $\Gamma$. Note that, by the definition, $\eta(\alpha, 0)=$ $\eta(0, \alpha)=1$ and

$$
\eta(\alpha, \beta+\gamma)=\eta(\alpha, \beta) \cdot \eta(\alpha, \gamma) \text { and } \eta(\alpha+\beta, \gamma)=\eta(\alpha, \gamma) \cdot \eta(\beta, \gamma)
$$

for $\alpha, \beta, \gamma \in \Gamma$.

Lemma 2.4. For $\alpha, \beta \in \Gamma_{\geq 0}$, we have a commutative diagram:

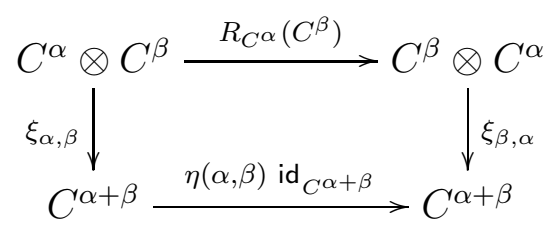

Proof. For $\alpha=\sum_{i \in I} a_{i} e_{i}$, set ht $(\alpha)=\sum_{i \in I} a_{i}$. We shall show the assertion by induction on $\operatorname{ht}(\alpha)$. It is trivial when either $\alpha=0$ or $\beta=0$.

First we assume ht $(\alpha)>1$. Then write $\alpha=\alpha^{\prime}+\alpha^{\prime \prime}$ with $\alpha^{\prime}, \alpha^{\prime \prime} \neq 0$. Setting $C^{\beta_{1}, \ldots, \beta_{n}}=C^{\beta_{1}} \otimes \cdots \otimes C^{\beta_{n}}$, the assertion follows from the following commutative diagram:

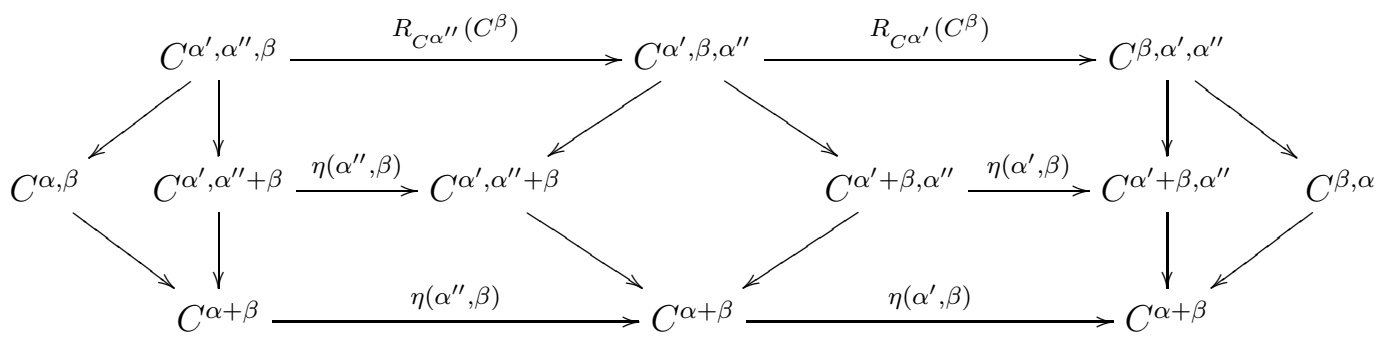

We next assume $\operatorname{ht}(\alpha)=1$. If $\operatorname{ht}(\beta)=1$, then it's done by the definition of $\eta_{i, j}$. When $\operatorname{ht}(\beta)>1$, the assertion follows by arguing similarly by induction on $\operatorname{ht}(\beta)$.

\subsection{Localizations.}

Let $\left(C_{i}, R_{C_{i}}\right)_{i \in I}$ be a real commuting family of braiders in $\mathcal{T}$. We define an order $\preceq$ on $\Gamma$ by

$$
\alpha \preceq \beta \quad \text { for } \alpha, \beta \in \Gamma \text { with } \beta-\alpha \in \Gamma_{\geq 0}
$$

Note that $\preceq$ is directed, i.e., for $\alpha, \beta \in \Gamma$, there exists $\gamma \in \Gamma$ such that $\alpha \preceq \gamma$ and $\beta \preceq \gamma$. For $\alpha_{1}, \ldots, \alpha_{k} \in \Gamma$, we set

$$
\mathcal{D}_{\alpha_{1}, \ldots, \alpha_{k}}:=\left\{\delta \in \Gamma \mid \alpha_{i}+\delta \in \Gamma_{\geq 0} \text { for any } i=1, \ldots, k\right\} .
$$

For $X, Y \in \mathcal{T}$ and $\delta, \delta^{\prime} \in \mathcal{D}_{\alpha, \beta}$ with $\delta \preceq \delta^{\prime}$, we define the map

$$
\zeta_{\delta^{\prime}, \delta}: \operatorname{Hom}_{\mathcal{T}}\left(C^{\delta+\alpha} \otimes X, Y \otimes C^{\delta+\beta}\right) \rightarrow \operatorname{Hom}_{\mathcal{T}}\left(C^{\delta^{\prime}+\alpha} \otimes X, Y \otimes C^{\delta^{\prime}+\beta}\right)
$$


by sending $f$ to $\zeta_{\delta^{\prime}, \delta}(f)$ such that the following diagram commutes:

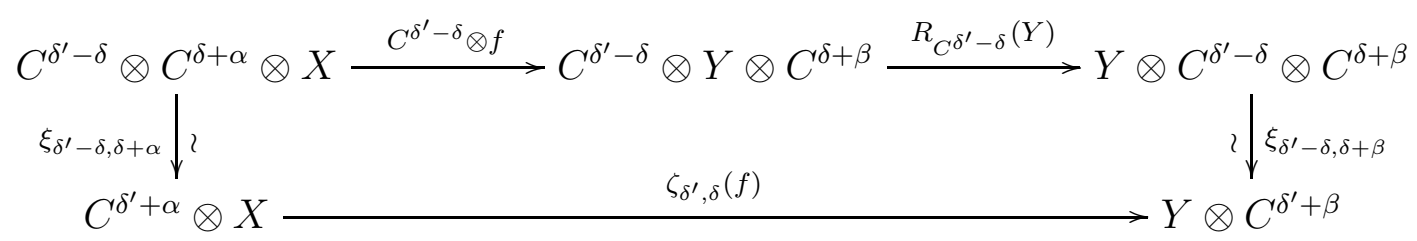

For $\delta, \delta^{\prime}, \delta^{\prime \prime} \in \mathcal{D}_{\alpha, \beta}$ with $\delta \preceq \delta^{\prime} \preceq \delta^{\prime \prime}$, it follows from (1.5) and (2.3) that the following diagram

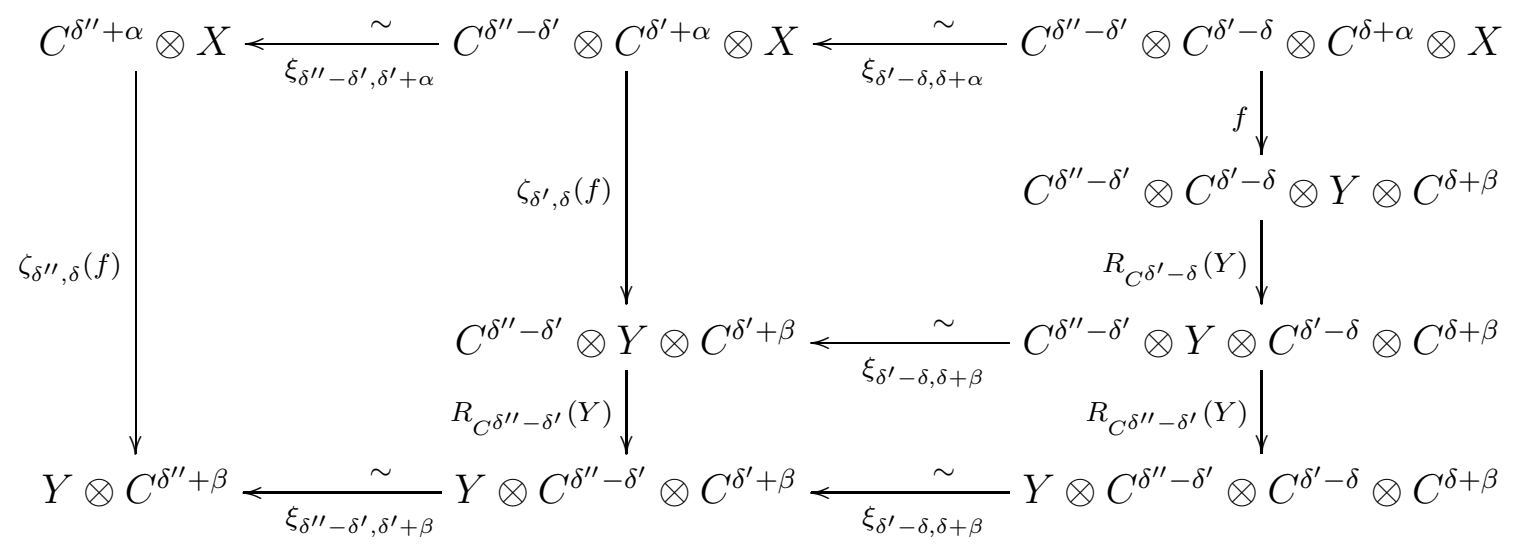

commutes, which implies that

$$
\zeta_{\delta^{\prime \prime}, \delta^{\prime}} \circ \zeta_{\delta^{\prime}, \delta}=\zeta_{\delta^{\prime \prime}, \delta}
$$

Thus, it becomes an inductive system (or direct system) on $\mathcal{D}_{\alpha, \beta}$.

We shall define a localization $\widetilde{\mathcal{T}}$ of $\mathcal{T}$ by $\left(C_{i}, R_{C_{i}}\right)_{i \in I}$ as follows. We set

$$
\begin{aligned}
\operatorname{Ob}(\widetilde{\mathcal{T}}) & :=\operatorname{Ob}(\mathcal{T}) \times \Gamma \\
\operatorname{Hom}_{\tilde{\mathcal{T}}}((X, \alpha),(Y, \beta)) & :=\lim _{\delta \in \overrightarrow{\mathcal{D}}_{\alpha, \beta}} \mathrm{H}_{\delta}((X, \alpha),(Y, \beta)),
\end{aligned}
$$

where

$$
\mathrm{H}_{\delta}((X, \alpha),(Y, \beta)):=\operatorname{Hom}_{\mathcal{T}}\left(C^{\delta+\alpha} \otimes X, Y \otimes C^{\delta+\beta}\right) .
$$

Here, the inductive limit is taken over the inductive system on $\mathcal{D}_{\alpha, \beta}$ defined as above.

For $f \in \mathrm{H}_{\delta}((X, \alpha),(Y, \beta))$ and $g \in \mathrm{H}_{\epsilon}((Y, \beta),(Z, \gamma))$, we define

$$
\Psi_{\delta, \epsilon}(f, g):=\eta(\delta+\beta, \beta-\gamma) \cdot \widetilde{\Psi}_{\delta, \epsilon}(f, g) \in \mathrm{H}_{\delta+\epsilon+\beta}((X, \alpha),(Z, \gamma))
$$


where $\eta$ is given in (2.4) and $\widetilde{\Psi}_{\delta, \epsilon}(f, g)$ is the morphism such that the following diagram commutes:

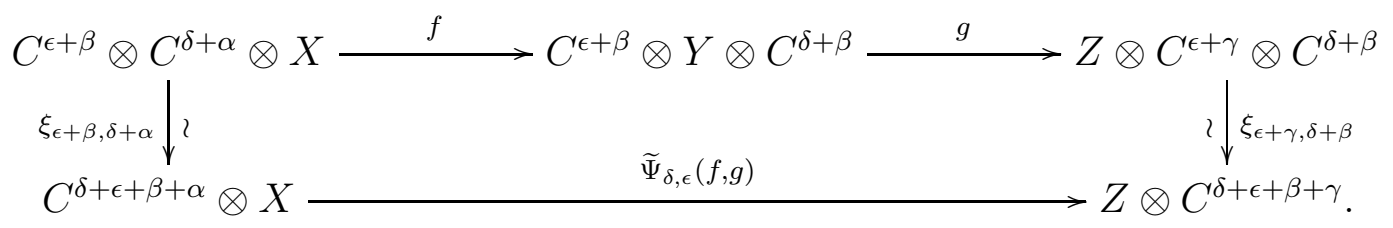

We set

$$
C^{\alpha_{1}, \ldots, \alpha_{t}}:=C^{\alpha_{1}} \otimes \cdots \otimes C^{\alpha_{t}}
$$

for $\alpha_{1}, \ldots \alpha_{t} \in \Gamma_{\geq 0}$. Then, for $\delta^{\prime} \succeq \delta$ and $\epsilon^{\prime} \succeq \epsilon$, one can show that the following diagram commutes:

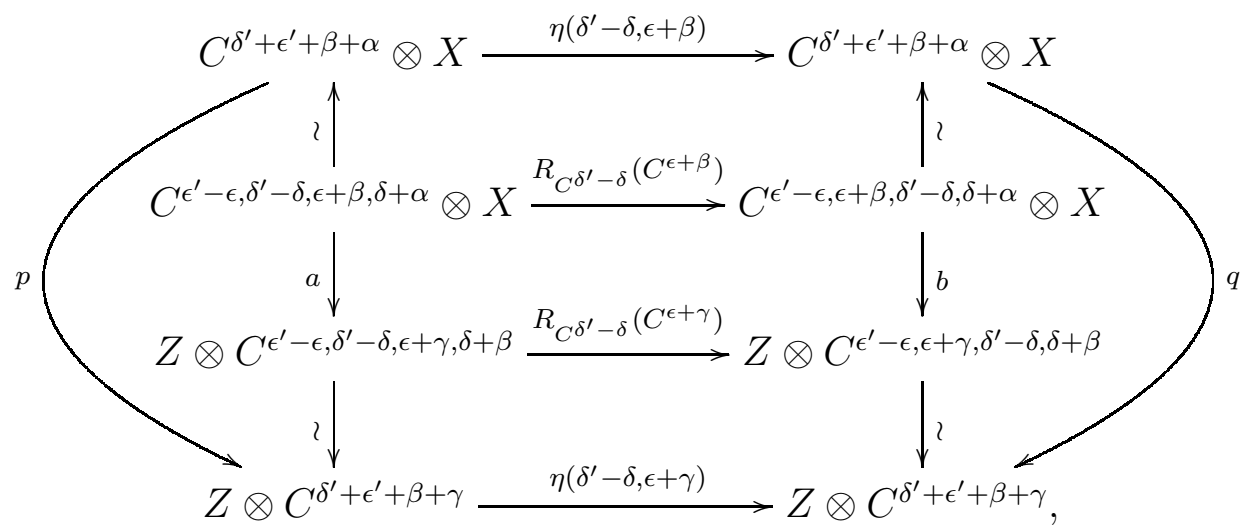

where

$$
\begin{array}{ll}
a=R_{C^{\epsilon^{\prime}-\epsilon, \delta^{\prime}-\delta}}(Z) \circ g \circ f, & b=R_{C^{\epsilon^{\prime}-\epsilon}}(Z) \circ g \circ R_{C^{\delta^{\prime}-\delta}}(Y) \circ f, \\
p=\zeta_{\delta^{\prime}+\epsilon^{\prime}+\beta, \delta+\epsilon+\beta}\left(\widetilde{\Psi}_{\delta, \epsilon}(f, g)\right), & q=\widetilde{\Psi}_{\delta^{\prime}, \epsilon^{\prime}}\left(\zeta_{\delta^{\prime}, \delta}(f), \zeta_{\epsilon^{\prime}, \epsilon}(g)\right),
\end{array}
$$

and other vertical morphisms are given by $\xi$ 's. Thus, by (2.5), we have

$$
\begin{aligned}
\Psi_{\delta^{\prime}, \epsilon^{\prime}}\left(\zeta_{\delta^{\prime}, \delta}(f), \zeta_{\epsilon^{\prime}, \epsilon}(g)\right) & =\eta\left(\delta^{\prime}+\beta, \beta-\gamma\right) \cdot \widetilde{\Psi}_{\delta^{\prime}, \epsilon^{\prime}}\left(\zeta_{\delta^{\prime}, \delta}(f), \zeta_{\epsilon^{\prime}, \epsilon}(g)\right) \\
& =\eta(\delta+\beta, \beta-\gamma) \cdot \zeta_{\delta^{\prime}+\epsilon^{\prime}+\beta, \delta+\epsilon+\beta}\left(\widetilde{\Psi}_{\delta, \epsilon}(f, g)\right) \\
& =\zeta_{\delta^{\prime}+\epsilon^{\prime}+\beta, \delta+\epsilon+\beta}\left(\Psi_{\delta, \epsilon}(f, g)\right),
\end{aligned}
$$

which tells that the following diagram commutes:

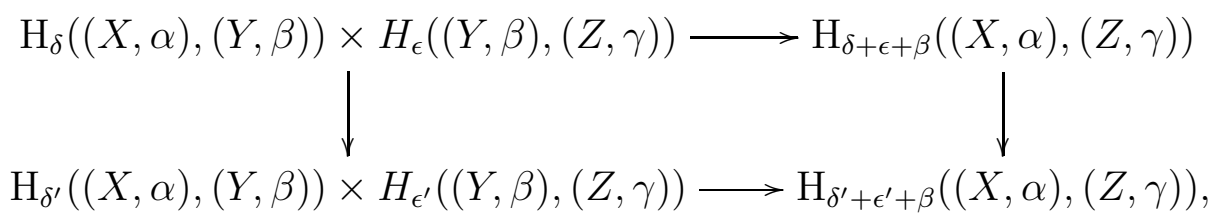


where the horizontal maps are given by $\Psi$ 's and the vertical maps are given by $\zeta$ 's. This yields the composition

$$
\operatorname{Hom}_{\tilde{\mathcal{T}}}((X, \alpha),(Y, \beta)) \times \operatorname{Hom}_{\widetilde{\mathcal{T}}}((Y, \beta),(Z, \gamma)) \rightarrow \operatorname{Hom}_{\widetilde{\mathcal{T}}}((X, \alpha),(Z, \gamma))
$$

in $\tilde{\mathcal{T}}$. We can check also the associativity, and we conclude that $\tilde{\mathcal{T}}$ is a category. Note that the identity id $\in \operatorname{End}_{\widetilde{\mathcal{T}}}((X, \alpha))$ is induced from the identity

$$
\operatorname{id}_{X} \in \operatorname{Hom}_{\mathcal{T}}(X, X)=\mathrm{H}_{-\alpha}((X, \alpha),(X, \alpha)) .
$$

We shall define a bifunctor $\otimes$ on the category $\widetilde{\mathcal{T}}$. For $\alpha, \beta \in \Gamma$ and $X, Y \in \mathcal{T}$, we set

$$
(X, \alpha) \otimes(Y, \beta):=(X \otimes Y, \alpha+\beta) .
$$

To define a tensor of morphisms, for $f \in \mathrm{H}_{\delta}\left((X, \alpha),\left(X^{\prime}, \alpha^{\prime}\right)\right)$ and $g \in \mathrm{H}_{\epsilon}\left((Y, \beta),\left(Y^{\prime}, \beta^{\prime}\right)\right)$ we define

$$
T_{\delta, \epsilon}(f, g):=\eta\left(\epsilon, \alpha-\alpha^{\prime}\right) \widetilde{T}_{\delta, \epsilon}(f, g) \in \mathrm{H}_{\delta+\epsilon}\left((X \otimes Y, \alpha+\beta),\left(X^{\prime} \otimes Y^{\prime}, \alpha^{\prime}+\beta^{\prime}\right)\right),
$$

where $\widetilde{T}_{\delta, \epsilon}(f, g)$ is the morphism such that the following diagram commutes:

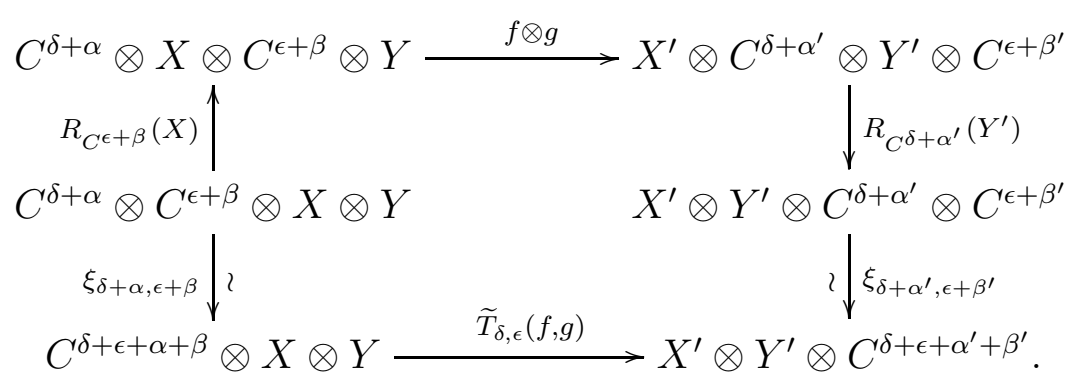

Then, for $\delta^{\prime} \succeq \delta$ and $\epsilon^{\prime} \succeq \epsilon$, one can show that

$$
\zeta_{\delta^{\prime}+\epsilon, \delta+\epsilon}\left(\widetilde{T}_{\delta, \epsilon}(f, g)\right)=\widetilde{T}_{\delta^{\prime}, \epsilon}\left(\zeta_{\delta^{\prime}, \delta}(f), g\right)
$$

and the following diagram commutes:

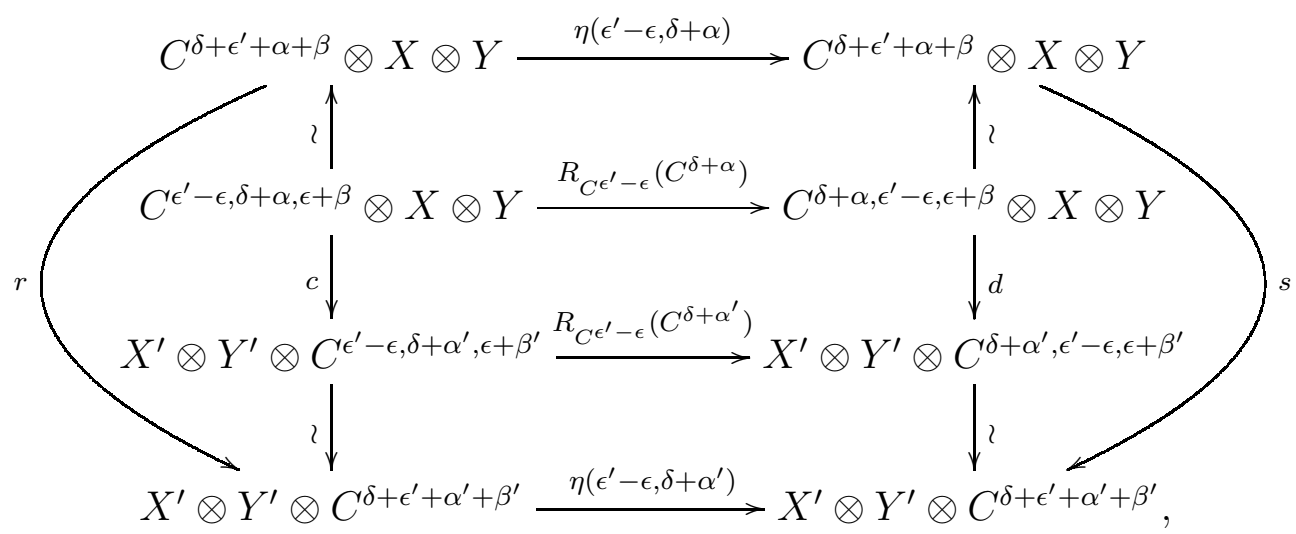


where

$$
\begin{aligned}
& c=R_{C^{\epsilon^{\prime}-\epsilon, \delta+\alpha^{\prime}}}\left(Y^{\prime}\right) \circ R_{C^{\epsilon^{\prime}-\epsilon}}\left(X^{\prime}\right) \circ(f \otimes g) \circ R_{C^{\epsilon+\beta}}(X), \\
& d=R_{C^{\delta+\alpha^{\prime}, \epsilon^{\prime}-\epsilon}}\left(Y^{\prime}\right) \circ(f \otimes g) \circ R_{C^{\epsilon^{\prime}-\epsilon, \epsilon+\beta}}(X), \\
& r=\zeta_{\delta+\epsilon^{\prime}, \delta+\epsilon}\left(\widetilde{T}_{\delta, \epsilon}(f, g)\right), \quad s=\widetilde{T}_{\delta, \epsilon^{\prime}}\left(f, \zeta_{\epsilon^{\prime}, \epsilon}(g)\right),
\end{aligned}
$$

and other vertical morphisms are given by $\xi$ 's. Thus, by (2.5), we have

$$
\begin{aligned}
T_{\delta^{\prime}, \epsilon^{\prime}} & \left(\zeta_{\delta^{\prime}, \delta}(f), \zeta_{\epsilon^{\prime}, \epsilon}(g)\right) \\
& =\eta\left(\epsilon^{\prime}, \alpha-\alpha^{\prime}\right) \cdot \widetilde{T}_{\delta^{\prime}, \epsilon^{\prime}}\left(\zeta_{\delta^{\prime}, \delta}(f), \zeta_{\epsilon^{\prime}, \epsilon}(g)\right) \\
& =\eta\left(\epsilon^{\prime}, \alpha-\alpha^{\prime}\right) \eta\left(\epsilon^{\prime}-\epsilon, \delta+\alpha\right)^{-1} \eta\left(\epsilon^{\prime}-\epsilon, \delta+\alpha^{\prime}\right) \cdot \zeta_{\delta^{\prime}+\epsilon^{\prime}, \delta+\epsilon}\left(\widetilde{T}_{\delta, \epsilon}(f, g)\right) \\
& =\zeta_{\delta^{\prime}+\epsilon^{\prime}, \delta+\epsilon}\left(T_{\delta, \epsilon}(f, g)\right) .
\end{aligned}
$$

Therefore, for $f \in \operatorname{Hom}_{\tilde{\mathcal{T}}}\left((X, \alpha),\left(X^{\prime}, \alpha^{\prime}\right)\right)$ and $g \in \operatorname{Hom}_{\widetilde{\mathcal{T}}}\left((Y, \beta),\left(Y^{\prime}, \beta^{\prime}\right)\right)$, we have the tensor product

$$
f \otimes g \in \operatorname{Hom}_{\tilde{\mathcal{T}}}\left((X, \alpha) \otimes(Y, \beta),\left(X^{\prime}, \alpha^{\prime}\right) \otimes\left(Y^{\prime}, \beta^{\prime}\right)\right)
$$

induced from $T_{\delta, \epsilon}$ over the inductive system.

Proposition 2.5. Let $f_{k} \in \mathrm{H}_{\delta_{k}}\left(\left(X_{k}, \alpha_{k}\right),\left(Y_{k}, \beta_{k}\right)\right)$ and $g_{k} \in \mathrm{H}_{\epsilon_{k}}\left(\left(Y_{k}, \beta_{k}\right),\left(Z_{k}, \gamma_{k}\right)\right)$ for $k=1,2$. Then, we have

$$
\Psi_{\delta_{1}+\delta_{2}, \epsilon_{1}+\epsilon_{2}}\left(T_{\delta_{1}, \delta_{2}}\left(f_{1}, f_{2}\right), T_{\epsilon_{1}, \epsilon_{2}}\left(g_{1}, g_{2}\right)\right)=T_{\delta_{1}+\epsilon_{1}+\beta_{1}, \delta_{2}+\epsilon_{2}+\beta_{2}}\left(\Psi_{\delta_{1}, \epsilon_{1}}\left(f_{1}, g_{1}\right), \Psi_{\delta_{2}, \epsilon_{2}}\left(f_{2}, g_{2}\right)\right)
$$

Proof. By Lemma 2.4, we have the commutative diagram

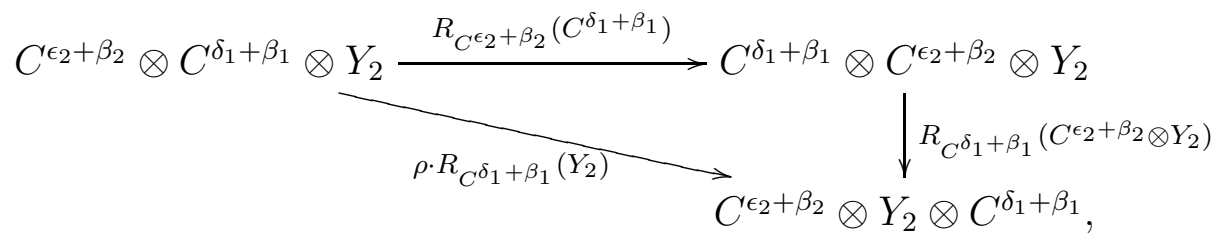


where $\rho=\eta\left(\delta_{1}+\beta_{1}, \epsilon_{2}+\beta_{2}\right) \eta\left(\epsilon_{2}+\beta_{2}, \delta_{1}+\beta_{1}\right)$. Then, one can prove that the following diagram commutes.

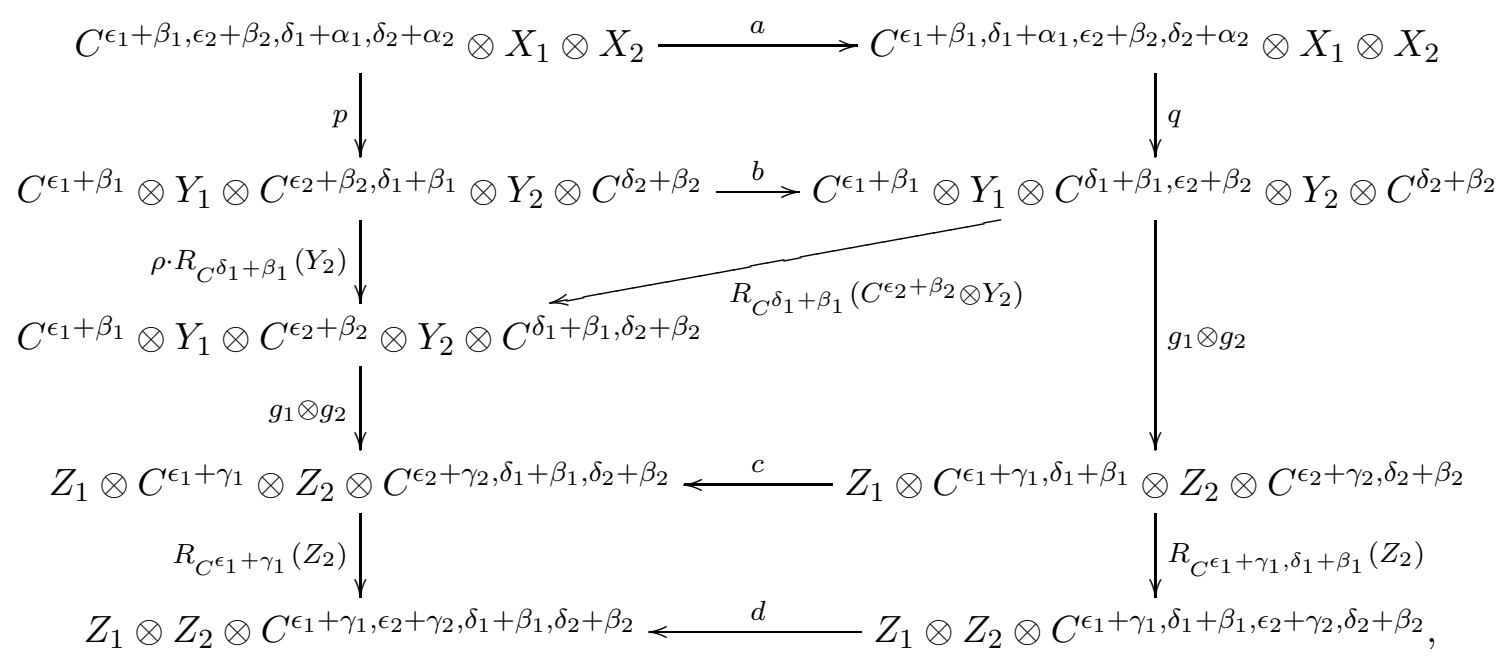

where

$$
\begin{array}{lll}
a=R_{C^{\epsilon_{2}+\beta_{2}}}\left(C^{\delta_{1}+\alpha_{1}}\right), & b=R_{C^{\epsilon_{2}+\beta_{2}}}\left(C^{\delta_{1}+\beta_{1}}\right), \\
c=R_{C^{\delta_{1}+\beta_{1}}}\left(Z_{2} \otimes C^{\epsilon_{2}+\gamma_{2}}\right), & d=R_{C^{\delta_{1}+\beta_{1}}}\left(C^{\epsilon_{2}+\gamma_{2}}\right), \\
p=R_{C^{\epsilon_{2}+\beta_{2}}}\left(Y_{1}\right) \circ\left(f_{1} \otimes f_{2}\right) \circ R_{C^{\delta_{2}+\alpha_{2}}}\left(X_{1}\right), & q=\left(f_{1} \otimes f_{2}\right) \circ R_{C^{\epsilon_{2}+\beta_{2}, \delta_{2}+\alpha_{2}}}\left(X_{1}\right) .
\end{array}
$$

We simply write

$$
\begin{aligned}
\widetilde{\Psi}(\widetilde{T}, \widetilde{T}) & :=\widetilde{\Psi}_{\delta_{1}+\delta_{2}, \epsilon_{1}+\epsilon_{2}}\left(\widetilde{T}_{\delta_{1}, \delta_{2}}\left(f_{1}, f_{2}\right), \widetilde{T}_{\epsilon_{1}, \epsilon_{2}}\left(g_{1}, g_{2}\right)\right) \\
\widetilde{T}(\widetilde{\Psi}, \widetilde{\Psi}) & :=\widetilde{T}_{\delta_{1}+\epsilon_{1}+\beta_{1}, \delta_{2}+\epsilon_{2}+\beta_{2}}\left(\widetilde{\Psi}_{\delta_{1}, \epsilon_{1}}\left(f_{1}, g_{1}\right), \widetilde{\Psi}_{\delta_{2}, \epsilon_{2}}\left(f_{2}, g_{2}\right)\right), \\
\widetilde{C} & :=C^{\delta_{1}+\delta_{2}+\epsilon_{1}+\epsilon_{2}+\beta_{1}+\beta_{2}+\alpha_{1}+\alpha_{2}}
\end{aligned}
$$

Then, it follows from the above diagram that the following diagram commutes:

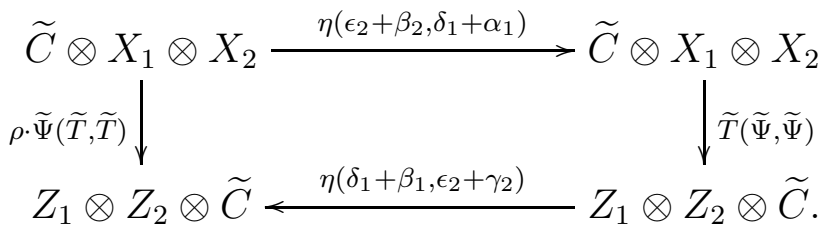


Since

$$
\begin{aligned}
& \Psi_{\delta_{1}+\delta_{2}, \epsilon_{1}+\epsilon_{2}}\left(T_{\delta_{1}, \delta_{2}}\left(f_{1}, f_{2}\right), T_{\epsilon_{1}, \epsilon_{2}}\left(g_{1}, g_{2}\right)\right) \\
& \quad=\eta\left(\delta_{1}+\delta_{2}+\beta_{1}+\beta_{2}, \beta_{1}+\beta_{2}-\gamma_{1}-\gamma_{2}\right) \eta\left(\delta_{2}, \alpha_{1}-\beta_{1}\right) \eta\left(\epsilon_{2}, \beta_{1}-\gamma_{1}\right) \widetilde{\Psi}(\widetilde{T}, \widetilde{T}), \\
& T_{\delta_{1}+\epsilon_{1}+\beta_{1}, \delta_{2}+\epsilon_{2}+\beta_{2}}\left(\Psi_{\delta_{1}, \epsilon_{1}}\left(f_{1}, g_{1}\right), \Psi_{\delta_{2}, \epsilon_{2}}\left(f_{2}, g_{2}\right)\right) \\
& \quad=\eta\left(\delta_{2}+\epsilon_{2}+\beta_{2}, \alpha_{1}-\gamma_{1}\right) \eta\left(\delta_{1}+\beta_{1}, \beta_{1}-\gamma_{1}\right) \eta\left(\delta_{2}+\beta_{2}, \beta_{2}-\gamma_{2}\right) \widetilde{T}(\widetilde{\Psi}, \widetilde{\Psi}),
\end{aligned}
$$

the assertion follows from (2.5) and (2.8).

By Proposition 2.5, we conclude that the tensor product (2.7) is a bifunctor

$$
\begin{aligned}
\operatorname{Hom}_{\tilde{\mathcal{T}}}\left((X, \alpha),\left(X^{\prime}, \alpha^{\prime}\right)\right) \times & \operatorname{Hom}_{\widetilde{\mathcal{T}}}\left((Y, \beta),\left(Y^{\prime}, \beta^{\prime}\right)\right) \\
& \longrightarrow \operatorname{Hom}_{\widetilde{\mathcal{T}}}\left((X, \alpha) \otimes(Y, \beta),\left(X^{\prime}, \alpha^{\prime}\right) \otimes\left(Y^{\prime}, \beta^{\prime}\right)\right) .
\end{aligned}
$$

Moreover, one can check that the composition of tensor products of morphisms satisfies the associativity, which tells that $\widetilde{\mathcal{T}}$ is a monoidal category. Note that $(\mathbf{1}, 0)$ is a unit of $\widetilde{\mathcal{T}}$.

Let us define the functor $\Upsilon: \mathcal{T} \rightarrow \widetilde{\mathcal{T}}$ by $X \mapsto(X, 0)$. It is easy to check that $\Upsilon$ is a monoidal functor.

Proposition 2.6. Let $\left(C_{i}, R_{C_{i}}\right)_{i \in I}$ be a real commuting family of braiders in a monoidal category $\mathcal{T}$. Then there is a real commuting family of braiders $\left(\widetilde{C}_{i}, R_{\widetilde{C}_{i}}\right)_{i \in I}$ in $\widetilde{\mathcal{T}}$ satisfy the following properties:

(i) for $i \in I, \Upsilon\left(C_{i}\right)$ is isomorphic to $\widetilde{C}_{i}$ and it is invertible in $(\widetilde{\mathcal{T}})_{\mathrm{br}}$,

(ii) for $i \in I$ and $X \in \mathcal{T}$, the diagram

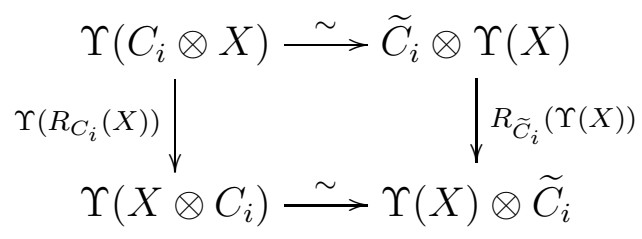

commutes.

Proof. For $\alpha \in \Gamma$, set $\widetilde{C}^{\alpha}=(\mathbf{1}, \alpha) \in \widetilde{\mathcal{T}}$. For $Z=(X, \beta) \in \widetilde{\mathcal{T}}$, define $R_{\widetilde{C}^{\alpha}}(Z): \widetilde{C}^{\alpha} \otimes Z \rightarrow$ $Z \otimes \widetilde{C}^{\alpha}$ as the image of $\operatorname{id}_{X} \in \operatorname{Hom}_{\mathcal{T}}(X, X)=\mathrm{H}_{-\alpha-\beta}\left(\widetilde{C}^{\alpha} \otimes Z, Z \otimes \widetilde{C}^{\alpha}\right)$ by the map $\mathrm{H}_{-\alpha-\beta}\left(\widetilde{C}^{\alpha} \otimes Z, Z \otimes \widetilde{C}^{\alpha}\right) \rightarrow \operatorname{Hom}_{\widetilde{\mathcal{T}}}\left(\widetilde{C}^{\alpha} \otimes Z, Z \otimes \widetilde{C}^{\alpha}\right)$. We can easily check that $\left(\widetilde{C}^{\alpha}, R_{\widetilde{C}^{\alpha}}\right)$ is a central braider and invertible in $(\widetilde{\mathcal{T}})_{\mathrm{br}}$.

Let us show that $\Upsilon\left(C^{\alpha}\right)$ is isomorphic to $\widetilde{C}^{\alpha}$ for $\alpha \in \Gamma_{\geq 0}$. 
Define $f:\left(C^{\alpha}, 0\right) \rightarrow \widetilde{C}^{\alpha}=(\mathbf{1}, \alpha)$ by $\operatorname{id}_{C^{\alpha}} \in \operatorname{Hom}_{\mathcal{T}}\left(C^{\alpha}, C^{\alpha}\right)=\mathrm{H}_{0}\left(\left(C^{\alpha}, 0\right),(\mathbf{1}, \alpha)\right)$, and $g: \widetilde{C}^{\alpha}=(\mathbf{1}, \alpha) \rightarrow\left(C^{\alpha}, 0\right)$ by $\operatorname{id}_{C^{\alpha}} \in \operatorname{Hom}_{\mathcal{T}}\left(C^{\alpha}, C^{\alpha}\right)=\mathrm{H}_{0}\left((\mathbf{1}, \alpha),\left(C^{\alpha}, 0\right)\right)$. Then one can see easily that $f$ and $g$ are inverse to each other.

Hence (i) and (ii) follow.

Theorem 2.7. Let $\left(C_{i}, R_{C_{i}}\right)_{i \in I}$ be a real commuting family of braiders in a monoidal category $\mathcal{T}$. There exist a monoidal category $\widetilde{\mathcal{T}}$ and a monoidal functor $\Upsilon: \mathcal{T} \rightarrow \widetilde{\mathcal{T}}$ such that

(i) $\Upsilon\left(C_{i}\right)$ is invertible in $\widetilde{\mathcal{T}}$ for any $i \in I$.

(ii) for any $i \in I$ and $X \in \mathcal{T}, \Upsilon\left(R_{C_{i}}(X)\right): \Upsilon\left(C_{i} \otimes X\right) \rightarrow \Upsilon\left(X \otimes C_{i}\right)$ is an isomorphism.

Moreover $\Upsilon$ satisfies the following universal property.

(iii) If there are another monoidal category $\mathcal{T}^{\prime}$ and a monoidal functor $\Upsilon^{\prime}: \mathcal{T} \rightarrow \mathcal{T}^{\prime}$ such that the properties (i) and (ii) above hold for $\Upsilon^{\prime}$, then there exists a monoidal functor $F$, which is unique up to a unique isomorphism, such that the diagram

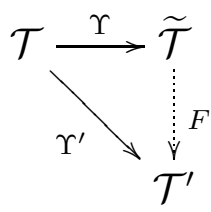

commutes.

We denote by $\mathcal{T}\left[C_{i}^{\otimes-1} \mid i \in I\right]$ the localization $\widetilde{\mathcal{T}}$ given in Theorem 2.7.

Proof. Let $\widetilde{\mathcal{T}}$ and $\Upsilon: \mathcal{T} \rightarrow \widetilde{\mathcal{T}}$ be the monoidal category and the functor that we have constructed. Then it satisfies (i) and (ii) by Proposition 2.6.

Let us show (iii). Set $\widetilde{C}_{i}=\Upsilon^{\prime}\left(C_{i}\right)$. Then, it forms a commuting family of invertible objects in $\mathcal{T}^{\prime}$, and there exist a family $\left\{\widetilde{C}^{\alpha}\right\}_{\alpha \in \Gamma}$ and isomorphism $\tilde{\xi}_{\alpha, \beta}: \widetilde{C}^{\alpha} \otimes$ $\widetilde{C}^{\beta} \sim \widetilde{C}^{\alpha+\beta}$ such that $\Upsilon^{\prime}\left(C^{\alpha}\right) \simeq \widetilde{C}^{\alpha}$ for $\alpha \in \Gamma_{\geq 0}$.

Now let us define the functor $F: \tilde{\mathcal{T}} \rightarrow \widetilde{\mathcal{T}}^{\prime}$. Set $F((X, \alpha))=\widetilde{C}^{\alpha} \otimes \Upsilon^{\prime}(X)$ for $X \in \mathcal{T}$ and $\alpha \in \Gamma$. Let $(X, \alpha),(Y, \beta) \in \widetilde{\mathcal{T}}$ and $\delta \in \mathcal{D}_{\alpha, \beta}$. For $f \in \mathrm{H}_{\delta}((X, \alpha),(Y, \beta))=$ $\operatorname{Hom}_{\mathcal{T}}\left(C^{\delta+\alpha} \otimes X, Y \otimes C^{\delta+\beta}\right)$, we define $F(f) \in \operatorname{Hom}_{\widetilde{\mathcal{T}}^{\prime}}(F((X, \alpha)), F((Y, \beta)))$ by the following commutative diagram

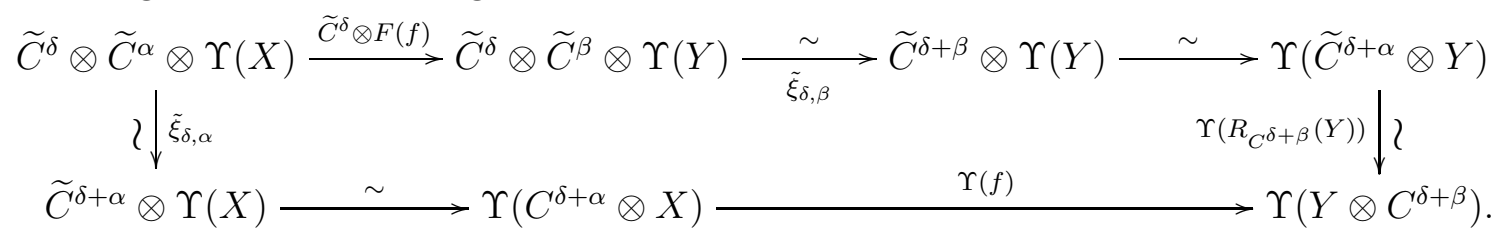


Then it is easy to see that $F$ is a well defined functor and satisfies the desired properties.

Lemma 2.8. Let $\left(C_{i}, R_{C_{i}}\right)_{i \in I}$ be a real commuting family of braiders in a monoidal category $\mathcal{T}$, and assume that $I$ is a finite set. Assume that $\alpha \in \Gamma$ satisfies $\alpha-e_{i} \in \Gamma_{\geq 0}$ for any $i \in I$. Then $\mathcal{T}\left[\left(C^{\alpha}\right)^{\otimes-1}\right]$ is equivalent to $\mathcal{T}\left[C_{i}^{\otimes-1} \mid i \in I\right]$.

Proof. It is enough to show that $\mathcal{T}^{\prime}:=\mathcal{T}\left[\left(C^{\alpha}\right)^{\otimes-1}\right]$ satisfies properties (i), (ii) in Theorem 2.7. It is obvious that $\left(C^{\alpha}\right)^{\otimes-1} \otimes C^{\alpha-e_{i}}$ is an inverse of $C_{i}$ in $\mathcal{T}^{\prime}$, and hence (i) holds.

For any $X \in \mathcal{T}$, the morphism $R_{C^{\alpha}}(X)=\left(R_{C^{\alpha-e_{i}}}(X) \otimes C_{i}\right) \circ\left(C^{\alpha-e_{i}} \otimes R_{C_{i}}(X)\right)$ is invertible in $\mathcal{T}^{\prime}$, and hence $C^{\alpha-e_{i}} \otimes R_{C_{i}}(X)$ has a left inverse. Since $C^{\alpha-e_{i}}$ is invertible, $R_{C_{i}}(X)$ has a left inverse. Similarly, $R_{C_{i}}(X)$ has a right inverse.

Proposition 2.9 (cf. [12, Proposition A.8]). Let $\left(C_{i}, R_{C_{i}}\right)_{i \in I}$ be a real commuting family of braiders in a monoidal category $\mathcal{T}$, and set $\widetilde{\mathcal{T}}:=\mathcal{T}\left[C_{i}^{\otimes-1} \mid i \in I\right]$. Assume that

(a) $\mathcal{T}$ is an abelian category,

(b) $\otimes$ is exact.

Then $\widetilde{\mathcal{T}}$ is an abelian category in which $\otimes$ is exact, and the functor $\Upsilon: \mathcal{T} \rightarrow \widetilde{\mathcal{T}}$ is exact.

Proposition 2.10. Let $\left(C_{i}, R_{C_{i}}\right)_{i \in I}$ be a real commuting family of braiders in a monoidal category $\mathcal{T}$, and set $\widetilde{\mathcal{T}}:=\mathcal{T}\left[C_{i}^{\otimes-1} \mid i \in I\right]$. Assume that

(a) $\mathcal{T}$ is an abelian category,

(b) $\otimes$ is exact.

Let $\mathcal{T}^{\prime}$ be a full subcategory of $\mathcal{T}$ closed by taking subquotients, extensions, and contains all the $C_{i}$ 's, and let $\widetilde{\mathcal{T}}^{\prime}$ be the localization $\mathcal{T}^{\prime}\left[C_{i}^{\otimes-1} \mid i \in I\right]$. Then $\tilde{\mathcal{T}}^{\prime}$ is a full subcategory of $\widetilde{\mathcal{T}}$ closed by taking subquotients and extensions.

Proof. It is obvious that $\tilde{\mathcal{T}}^{\prime}$ is a full subcategory of $\tilde{\mathcal{T}}$ closed by taking subquotients. Let us show that $\tilde{\mathcal{T}}^{\prime}$ is closed by extensions.

Let us denote by $\Phi: \mathcal{T} \rightarrow \widetilde{\mathcal{T}}$ the canonical functor. Let $0 \rightarrow X^{\prime} \rightarrow X \rightarrow X^{\prime \prime} \rightarrow 0$ be an exact sequence in $\widetilde{\mathcal{T}}$ such that $X^{\prime}, X^{\prime \prime} \in \widetilde{\mathcal{T}}^{\prime}$. We may assume that there exist a sequence of morphisms $Z^{\prime} \stackrel{f}{\longrightarrow} Z \stackrel{g}{\longrightarrow} Z^{\prime \prime}$ in $\mathcal{T}$ with $Z^{\prime}, Z^{\prime \prime} \in \mathcal{T}^{\prime}$ and $\alpha \in \Gamma_{\geq 0}$ such that $\Phi\left(C^{\alpha}\right)^{\otimes-1} \otimes \Phi\left(Z^{\prime}\right) \rightarrow \Phi\left(C^{\alpha}\right)^{\otimes-1} \otimes \Phi(Z) \rightarrow \Phi\left(C^{\alpha}\right)^{\otimes-1} \otimes \Phi\left(Z^{\prime \prime}\right)$ is isomorphic to $X^{\prime} \rightarrow X \rightarrow X^{\prime \prime}$. Since $0 \rightarrow \operatorname{ker}(f) \rightarrow Z \rightarrow \operatorname{Im}(g) \rightarrow 0$ is exact, $Z$ belongs to $\mathcal{T}^{\prime}$. Hence $X \simeq \Phi\left(C^{\alpha}\right)^{\otimes-1} \otimes \Phi(Z)$ belongs to $\widetilde{\mathcal{T}}^{\prime}$. 
2.3. Graded cases. Let $\Lambda$ be a $\mathbb{Z}$-module. A k-linear monoidal category $\mathcal{T}$ is $\Lambda$-graded if $\mathcal{T}$ has a decomposition $\mathcal{T}=\bigoplus_{\lambda \in \Lambda} \mathcal{T}_{\lambda}$ such that $\mathbf{1} \in \mathcal{T}_{0}$ and $\otimes$ induces a bifunctor $\mathcal{T}_{\lambda} \times \mathcal{T}_{\mu} \rightarrow \mathcal{T}_{\lambda+\mu}$ for any $\lambda, \mu \in \Lambda$.

Let $\mathcal{T}$ be a $\Lambda$-graded monoidal category, and let $q$ be an invertible central braider in $\mathcal{T}$, which belongs to $\mathcal{T}_{0}$. We write $q^{n}(n \in \mathbb{Z})$ for $q^{\otimes n}$ for the sake of simplicity. A graded braider is a triple $\left(C, R_{C}, \phi\right)$ of an object $C$, an $\mathbb{Z}$-linear map $\phi: \Lambda \rightarrow \mathbb{Z}$ and a morphism

$$
R_{C}(X): C \otimes X \longrightarrow q^{\phi(\lambda)} \otimes X \otimes C
$$

which is functorial in $X \in \mathcal{T}_{\lambda}$ for $\lambda \in \Lambda$ such that a graded version of (2.1) holds. That means that the diagram

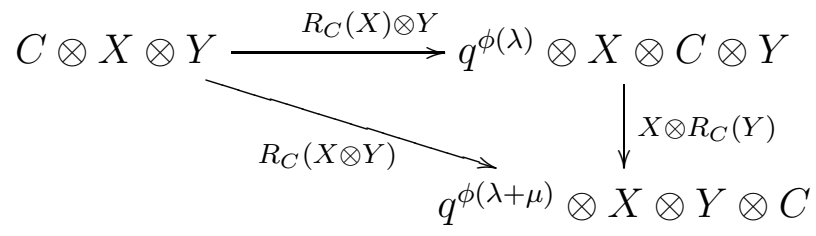

commutes for any $X \in \mathcal{T}_{\lambda}$ and $Y \in \mathcal{T}_{\mu}$.

In a similar manner, we can consider graded commuting family in a graded category $\mathcal{T}$. Let $I$ be an index set, and set $\Gamma=\mathbb{Z}^{\oplus I}$ and $\Gamma_{\geq 0}=\mathbb{Z}_{\geq 0}^{\oplus I}$. Let $P_{i}$ be an object for $i \in I$ and $B_{i, j}: P_{i} \otimes P_{j} \stackrel{\sim}{\longrightarrow} q^{\phi_{i, j}} \otimes P_{j} \otimes P_{i}$ an isomorphism for $i, j \in I$. We call a triple $\left(\left\{P_{i}\right\}_{i \in I},\left\{B_{i, j}\right\}_{i, j \in I},\left\{\phi_{i, j}\right\}_{i, j \in I}\right)$ a graded commuting family of $\mathcal{T}$ if it satisfies

(a) $\left\{\phi_{i, j}\right\}_{i, j \in I}$ is a $\mathbb{Z}$-valued skew-symmetric matrix,

(b) $B_{i, i}=\mathrm{id}_{P_{i} \otimes P_{i}}$ for $i \in I$,

(c) $B_{j, i} \circ B_{i, j}=\mathrm{id}_{P_{i} \otimes P_{j}}$ for $i, j \in I$,

(d) a graded version of (1.4) holds, i.e., for $i, j, k \in I$,

$$
\left(B_{j, k} \otimes P_{i}\right) \circ\left(P_{j} \otimes B_{i, k}\right) \circ\left(B_{i, j} \otimes P_{k}\right)=\left(P_{k} \otimes B_{i, j}\right) \circ\left(B_{i, k} \otimes P_{j}\right) \circ\left(P_{i} \otimes B_{j, k}\right) .
$$

Then we have a graded version of Lemma 1.16 .

Lemma 2.11. Let $\left(\left\{P_{i}\right\}_{i \in I},\left\{B_{i, j}\right\}_{i, j \in I},\left\{\phi_{i, j}\right\}_{i, j \in I}\right)$ be a graded commuting family in $\mathcal{T}$, and let $\mathrm{H}: \Gamma \otimes \Gamma \rightarrow \mathbb{Z}$ be a $\mathbb{Z}$-bilinear map such that $\phi_{i, j}=\mathrm{H}\left(e_{i}, e_{j}\right)-\mathrm{H}\left(e_{j}, e_{i}\right)$ for $i, j \in I$. Then there exist

(a) an object $P^{\alpha}$ for any $\alpha \in \Gamma_{\geq 0}$,

(b) an isomorphism $\xi_{\alpha, \beta}: P^{\alpha} \otimes P^{\beta} \stackrel{\sim}{\longrightarrow} q^{\mathrm{H}(\alpha, \beta)} \otimes P^{\alpha+\beta}$ for any $\alpha, \beta \in \Gamma_{\geq 0}$

such that

(i) $P^{0}=1$ and $P^{e_{i}}=P_{i}$ for $i \in I$, 
(ii) the diagram

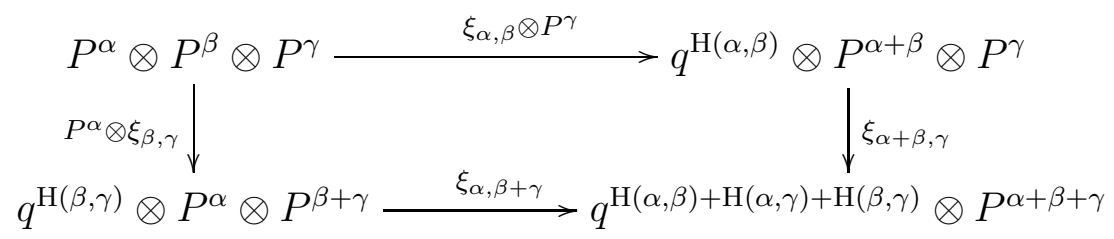

commutes for any $\alpha, \beta, \gamma \in \Gamma_{\geq 0}$,

(iii) the diagram
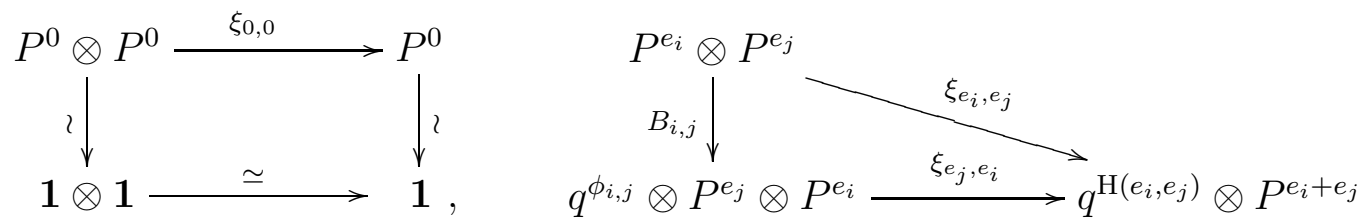

commutes for any $i, j \in I$.

Moreover, such a datum is unique up to a unique isomorphism.

We now consider a graded version of a real commuting family of braiders. Let $\left(C_{i}, R_{C_{i}}, \phi_{i}\right)$ be a graded braider for $i \in I$. We say that $\left(C_{i}, R_{C_{i}}, \phi_{i}\right)_{i \in I}$ is a real commuting family of graded braiders in $\mathcal{T}$ if

(a) $C_{i} \in \mathcal{T}_{\lambda_{i}}$ for some $\lambda_{i} \in \Lambda$, and $\phi_{i}\left(\lambda_{i}\right)=0, \phi_{i}\left(\lambda_{j}\right)+\phi_{j}\left(\lambda_{i}\right)=0$,

(b) $R_{C_{i}}\left(C_{i}\right) \in \mathbf{k}^{\times} \mathrm{id}_{C_{i} \otimes C_{i}}$ for $i \in I$,

(c) $R_{C_{j}}\left(C_{i}\right) \circ R_{C_{i}}\left(C_{j}\right) \in \mathbf{k}^{\times} \mathrm{id}_{C_{i} \otimes C_{j}}$ for $i, j \in I$.

We choose a $\mathbb{Z}$-bilinear map $\mathrm{H}: \Gamma \otimes \Gamma \rightarrow \mathbb{Z}$ such that $\phi_{i}\left(\lambda_{j}\right)=\mathrm{H}\left(e_{i}, e_{j}\right)-\mathrm{H}\left(e_{j}, e_{i}\right)$ for $i, j \in I$. Using the same argument of the proof of Lemma 2.3, by Lemma 2.11, we have $C^{\alpha}, \xi_{\alpha, \beta}$ and $\eta(\alpha, \beta)$ as in Section 2.1. We define a $\mathbb{Z}$-linear map

$$
\mathrm{L}: \Gamma \rightarrow \Lambda, \quad e_{i} \mapsto \lambda_{i} \text { for } i \in I,
$$

and a $\mathbb{Z}$-bilinear map

$$
\phi: \Gamma \times \Lambda \rightarrow \mathbb{Z}, \quad\left(e_{i}, \lambda\right) \mapsto \phi_{i}(\lambda)
$$

It is easy to check that

$$
\phi(\alpha, \mathrm{L}(\beta))=\mathrm{H}(\alpha, \beta)-\mathrm{H}(\beta, \alpha) \quad \text { for } \alpha, \beta \in \Gamma \text {. }
$$


Note that the diagram below commutes:

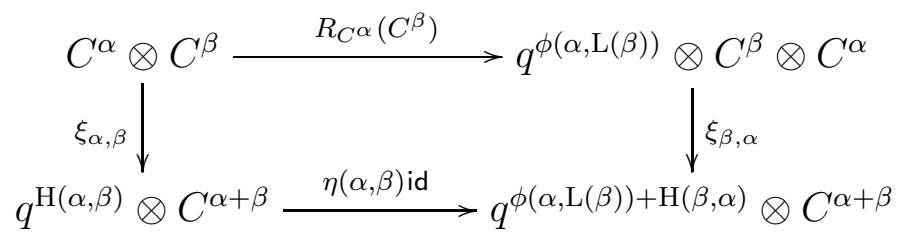

For $X \in \mathcal{T}_{\lambda}, Y \in \mathcal{T}_{\mu}$ and $\delta \in \mathcal{D}_{\alpha, \beta}$, we set

$$
\mathrm{H}_{\delta}^{\mathrm{gr}}((X, \alpha),(Y, \beta)):=\operatorname{Hom}_{\mathcal{T}}\left(C^{\delta+\alpha} \otimes X, q^{\mathrm{H}(\delta, \beta-\alpha)+\phi(\delta+\beta, \mu)} \otimes Y \otimes C^{\delta+\beta}\right) .
$$

For $\delta, \delta^{\prime} \in \mathcal{D}_{\alpha, \beta}$ with $\delta \preceq \delta^{\prime}$ and $f \in \mathrm{H}_{\delta}^{\mathrm{gr}}((X, \alpha),(Y, \beta))$, we define $\zeta_{\delta^{\prime}, \delta}^{\mathrm{gr}}(f)$ to be the morphism such that the following diagram commutes:

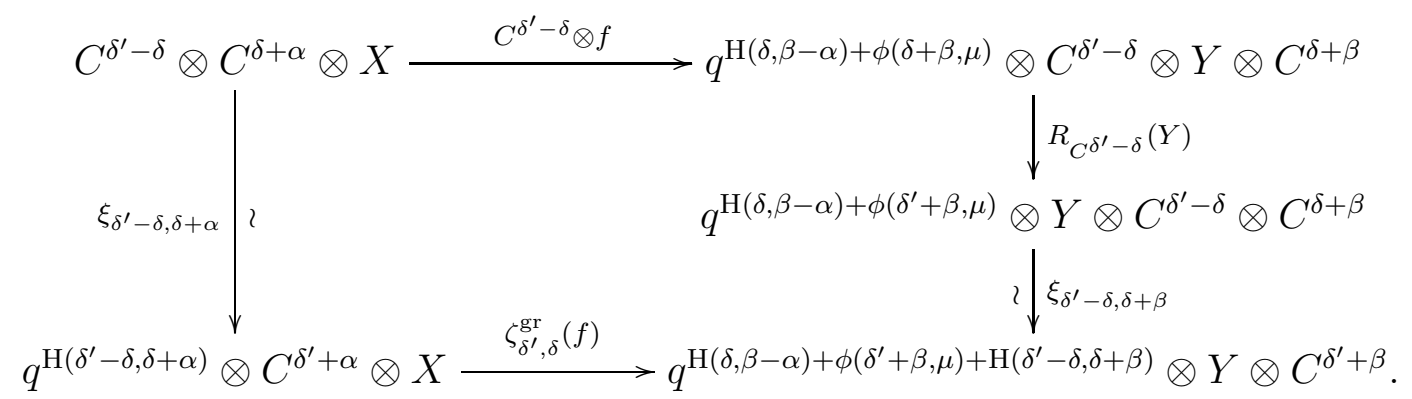

Since

$$
\begin{aligned}
& \mathrm{H}(\delta, \beta-\alpha)+\phi\left(\delta^{\prime}+\beta, \mu\right)+\mathrm{H}\left(\delta^{\prime}-\delta, \delta+\beta\right)-\mathrm{H}\left(\delta^{\prime}-\delta, \delta+\alpha\right) \\
& \quad=\mathrm{H}\left(\delta^{\prime}, \beta-\alpha\right)+\phi\left(\delta^{\prime}+\beta, \mu\right),
\end{aligned}
$$

we have the map

$$
\zeta_{\delta^{\prime}, \delta}^{\mathrm{gr}}: \mathrm{H}_{\delta}^{\mathrm{gr}}((X, \alpha),(Y, \beta)) \rightarrow \mathrm{H}_{\delta^{\prime}}^{\mathrm{gr}}((X, \alpha),(Y, \beta)) .
$$

One can show that $\zeta_{\delta^{\prime \prime}, \delta^{\prime}}^{\mathrm{gr}} \circ \zeta_{\delta^{\prime}, \delta}^{\mathrm{gr}}=\zeta_{\delta^{\prime \prime}, \delta}^{\mathrm{gr}}$ for $\delta \preceq \delta^{\prime} \preceq \delta^{\prime \prime}$, which tells that it becomes an inductive system.

As in Section 2.2 , we now obtain the category $\widetilde{\mathcal{T}}$ defined by

$$
\begin{aligned}
\operatorname{Ob}(\widetilde{\mathcal{T}}):=\operatorname{Ob}(\mathcal{T}) \times \Gamma, & \operatorname{Hom}_{\widetilde{\mathcal{T}}}((X, \alpha),(Y, \beta)):=\lim _{\substack{\delta \in \mathcal{D}_{\alpha, \beta}, \lambda+\mathrm{L}(\alpha)=\mu+\mathrm{L}(\beta)}} \mathrm{H}_{\delta}^{\mathrm{gr}}((X, \alpha),(Y, \beta)),
\end{aligned}
$$

where $X \in \mathcal{T}_{\lambda}$ and $Y \in \mathcal{T}_{\mu}$. By the construction, we have the decomposition

$$
\widetilde{\mathcal{T}}=\bigoplus_{\mu \in \Lambda} \widetilde{\mathcal{T}}_{\mu}, \quad \text { where } \widetilde{\mathcal{T}}_{\mu}:=\left\{(X, \alpha) \mid X \in \mathcal{T}_{\lambda}, \lambda+\mathrm{L}(\alpha)=\mu\right\}
$$


Let $X \in \mathcal{T}_{\lambda}, Y \in \mathcal{T}_{\mu}$ and $Z \in \mathcal{T}_{\nu}$. For $f \in \mathrm{H}_{\delta}^{\mathrm{gr}}((X, \alpha),(Y, \beta))$ and $g \in \mathrm{H}_{\epsilon}^{\mathrm{gr}}((Y, \beta),(Z, \gamma))$, we define

$$
\Psi_{\delta, \epsilon}^{\mathrm{gr}}(f, g):=\eta(\delta+\beta, \beta-\gamma) \cdot \widetilde{\Psi}_{\delta, \epsilon}^{\mathrm{gr}}(f, g),
$$

where $\widetilde{\Psi}_{\delta, \epsilon}^{\mathrm{gr}}(f, g)$ is the morphism such that the following diagram commutes:

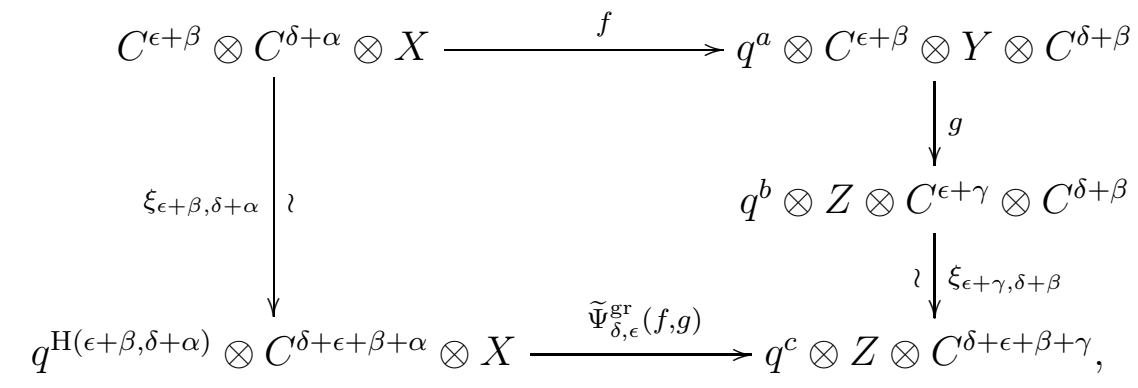

where

$$
\begin{aligned}
& a=\mathrm{H}(\delta, \beta-\alpha)+\phi(\delta+\beta, \mu), \quad b=a+\mathrm{H}(\epsilon, \gamma-\beta)+\phi(\epsilon+\gamma, \nu), \\
& c=b+\mathrm{H}(\epsilon+\gamma, \delta+\beta) .
\end{aligned}
$$

As $\mathrm{L}(\beta)+\mu=\mathrm{L}(\gamma)+\nu$, by $(2.9)$, we obtain

$$
\phi(\delta+\beta, \mu)=\mathrm{H}(\delta+\beta, \gamma-\beta)-\mathrm{H}(\gamma-\beta, \delta+\beta)+\phi(\delta+\beta, \nu) .
$$

This implies that

$$
\begin{aligned}
c & =\mathrm{H}(\delta, \beta-\alpha)+\phi(\delta+\beta, \mu)+\mathrm{H}(\epsilon, \gamma-\beta)+\phi(\epsilon+\gamma, \nu)+\mathrm{H}(\epsilon+\gamma, \delta+\beta) \\
& =\mathrm{H}(\delta+\epsilon+\beta, \gamma-\alpha)+\phi(\delta+\epsilon+\beta+\gamma, \nu)+\mathrm{H}(\epsilon+\beta, \delta+\alpha),
\end{aligned}
$$

which tells that

$$
\Psi_{\delta, \epsilon}^{\mathrm{gr}}(f, g) \in \mathrm{H}_{\delta+\epsilon+\beta}^{\mathrm{gr}}((X, \alpha),(Z, \gamma))
$$

Using the same argument as in Section 2.2, $\Psi_{\delta, \epsilon}^{\text {gr }}$ gives the composition in $\widetilde{\mathcal{T}}$, which means that $\widetilde{\mathcal{T}}$ is a category.

We shall define a bifunctor $\otimes$ on the category $\tilde{\mathcal{T}}$ as follows. For $\alpha, \alpha^{\prime}, \beta, \beta^{\prime} \in \Gamma$, $X \in \mathcal{T}_{\lambda}, X^{\prime} \in \mathcal{T}_{\lambda^{\prime}}, Y \in \mathcal{T}_{\mu}$ and $Y^{\prime} \in \mathcal{T}_{\mu^{\prime}}$, we define

$$
(X, \alpha) \otimes(Y, \beta):=\left(q^{-\phi(\beta, \lambda)+\mathrm{H}(\alpha, \beta)} \otimes X \otimes Y, \alpha+\beta\right),
$$

and, for $f \in \mathrm{H}_{\delta}^{\mathrm{gr}}\left((X, \alpha),\left(X^{\prime}, \alpha^{\prime}\right)\right)$ and $g \in \mathrm{H}_{\epsilon}^{\mathrm{gr}}\left((Y, \beta),\left(Y^{\prime}, \beta^{\prime}\right)\right)$, we define

$$
T_{\delta, \epsilon}^{\mathrm{gr}}(f, g):=\eta\left(\epsilon, \alpha-\alpha^{\prime}\right) \widetilde{T}_{\delta, \epsilon}^{\mathrm{gr}}(f, g),
$$


where $\widetilde{T}_{\delta, \epsilon}^{\mathrm{gr}}(f, g)$ is the morphism such that the following diagram commutes:

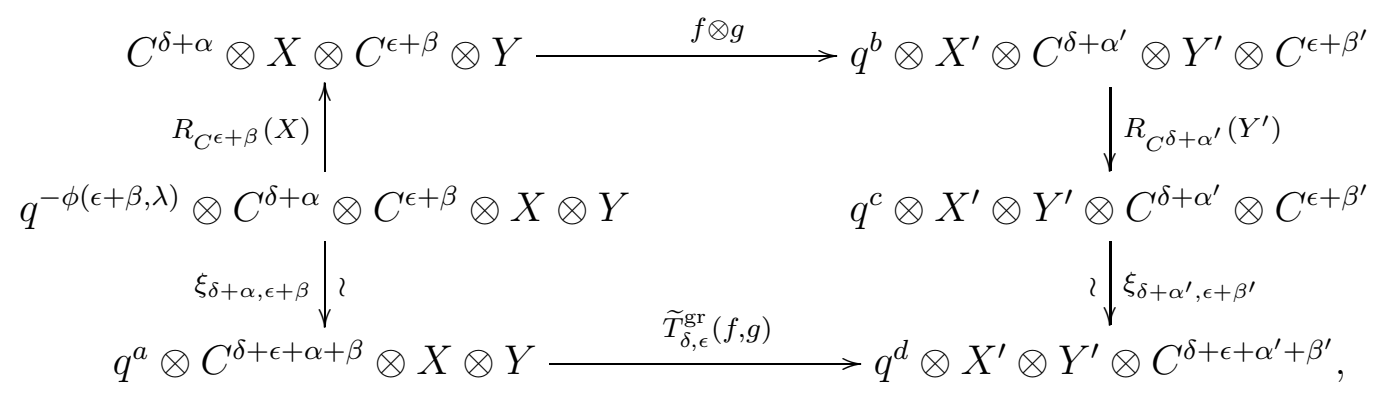

where

$$
\begin{aligned}
& a=-\phi(\epsilon+\beta, \lambda)+\mathrm{H}(\delta+\alpha, \epsilon+\beta), \\
& b=\mathrm{H}\left(\delta, \alpha^{\prime}-\alpha\right)+\phi\left(\delta+\alpha^{\prime}, \lambda^{\prime}\right)+\mathrm{H}\left(\epsilon, \beta^{\prime}-\beta\right)+\phi\left(\epsilon+\beta^{\prime}, \mu^{\prime}\right), \\
& c=b+\phi\left(\delta+\alpha^{\prime}, \mu^{\prime}\right), \quad d=c+\mathrm{H}\left(\delta+\alpha^{\prime}, \epsilon+\beta^{\prime}\right) .
\end{aligned}
$$

By $\lambda+\mathrm{L}(\alpha)=\lambda^{\prime}+\mathrm{L}\left(\alpha^{\prime}\right)$, we have

$$
\phi(\epsilon+\beta, \lambda)=\phi\left(\epsilon+\beta^{\prime}, \lambda^{\prime}\right)-\phi\left(\beta^{\prime}, \lambda^{\prime}\right)+\phi(\beta, \lambda)+\mathrm{H}\left(\epsilon, \alpha^{\prime}-\alpha\right)-\mathrm{H}\left(\alpha^{\prime}-\alpha, \epsilon\right),
$$

which implies that

$$
\begin{aligned}
d-a= & \mathrm{H}\left(\delta+\epsilon, \alpha^{\prime}+\beta^{\prime}-\alpha-\beta\right)+\phi\left(\epsilon+\delta+\alpha^{\prime}+\beta^{\prime}, \lambda^{\prime}+\mu^{\prime}\right) \\
& -\phi\left(\beta^{\prime}, \lambda^{\prime}\right)+\mathrm{H}\left(\alpha^{\prime}, \beta^{\prime}\right)+\phi(\beta, \lambda)-\mathrm{H}(\alpha, \beta) .
\end{aligned}
$$

This tells that

$$
T_{\delta, \epsilon}^{\mathrm{gr}}(f, g) \in \mathrm{H}_{\delta+\epsilon}^{\mathrm{gr}}\left((X, \alpha) \otimes(Y, \beta),\left(X^{\prime}, \alpha^{\prime}\right) \otimes\left(Y^{\prime}, \beta^{\prime}\right)\right) .
$$

By the same argument as in Section 2.2, one can show that $T_{\delta, \epsilon}^{\mathrm{gr}}$ yields a bifunctor

$$
\begin{aligned}
\operatorname{Hom}_{\tilde{\mathcal{T}}}\left((X, \alpha),\left(X^{\prime}, \alpha^{\prime}\right)\right) \times \operatorname{Hom}_{\tilde{\mathcal{T}}}\left((Y, \beta),\left(Y^{\prime}, \beta^{\prime}\right)\right) \stackrel{\otimes}{\longrightarrow} \\
\qquad \operatorname{Hom}_{\widetilde{\mathcal{T}}}\left((X, \alpha) \otimes(Y, \beta),\left(X^{\prime}, \alpha^{\prime}\right) \otimes\left(Y^{\prime}, \beta^{\prime}\right)\right),
\end{aligned}
$$

which gives the following theorem.

Theorem 2.12. $\widetilde{\mathcal{T}}$ becomes a monoidal category. Moreover, it satisfies the same conditions as in Theorem 2.7.

We denote by $\mathcal{T}\left[C_{i}^{\otimes-1} \mid i \in I\right]$ the localization $\widetilde{\mathcal{T}}$ in Theorem 2.12. Note that

$$
(X, \alpha+\beta) \simeq q^{-\mathrm{H}(\beta, \alpha)} \otimes\left(C^{\alpha} \otimes X, \beta\right), \quad(\mathbf{1}, \beta) \otimes(\mathbf{1},-\beta) \simeq q^{-\mathrm{H}(\beta, \beta)}(\mathbf{1}, 0)
$$

for $\alpha \in \Gamma_{\geq 0}$ and $\beta \in \Gamma$. We now have a graded version of Proposition 2.9 
Proposition 2.13. Let $\left(C_{i}, R_{C_{i}}, \phi_{i}\right)_{i \in I}$ be a real commuting family of graded braiders in a graded monoidal category $\mathcal{T}$, and set $\widetilde{\mathcal{T}}:=\mathcal{T}\left[C_{i}^{\otimes-1} \mid i \in I\right]$. Assume that

(a) $\mathcal{T}$ is an abelian category,

(b) $\otimes$ is exact,

Then $\widetilde{\mathcal{T}}$ is an abelian category with exact $\otimes$, and the functor $\Upsilon: \mathcal{T} \rightarrow \widetilde{\mathcal{T}}$ is exact.

\section{Affinizations And R-Matrices}

3.1. Affinizations. Let $R$ be a quiver Hecke algebra of arbitrary type. We recall the notions of affinizations and R-matrices given in [16]. For $\beta \in \mathrm{Q}_{+}$and $i \in I$, let

$$
\mathfrak{p}_{i, \beta}=\sum_{\nu \in I^{\beta}}\left(\prod_{a \in\{1, \ldots, \operatorname{ht}(\beta)\}, \nu_{a}=i} x_{a}\right) e(\nu) \in R(\beta) .
$$

Then $\mathfrak{p}_{i, \beta}$ belongs to the center of $R(\beta)$. When there is no afraid of confusion, we simply write $\mathfrak{p}_{i}$ for $\mathfrak{p}_{i, \beta}$.

Definition 3.1. Let $M$ be a simple $R(\beta)$-module. An affinization of $M$ with degree $d_{\widehat{\mathrm{M}}}$ is an $R(\beta)$-module $\widehat{\mathrm{M}}$ with an endomorphism $z_{\widehat{\mathrm{M}}}$ of $\widehat{\mathrm{M}}$ with degree $d_{\widehat{\mathrm{M}}} \in \mathbb{Z}_{>0}$ and an isomorphism $\widehat{\mathrm{M}} / z_{\widehat{\mathrm{M}}} \widehat{\mathrm{M}} \simeq M$ such that

(i) $\widehat{\mathrm{M}}$ is a finitely generated free module over the polynomial ring $\mathbf{k}\left[z_{\widehat{\mathrm{M}}}\right]$,

(ii) $\mathfrak{p}_{i} \widehat{\mathrm{M}} \neq 0$ for all $i \in I$.

Note that every affinization is essentially even, i.e. $d_{\widehat{M}} \in 2 \mathbb{Z}_{>0}[16$, Proposition 2.5]. Thus, from now on, we assume that every affinization is even.

Lemma 3.2 ([16, Lemma 2.7]). Let $\widehat{\mathrm{M}}$ be an affinization of a simple $R$-module $M$. Then we have

$$
\operatorname{END}_{R_{\mathbf{k}\left[z_{\widehat{M}}\right]}(\beta)}(\widehat{\mathrm{M}}) \simeq \mathbf{k}\left[z_{\widehat{\mathrm{M}}}\right] \mathrm{id}_{\widehat{\mathrm{M}}}
$$

In particular, $\left.\mathfrak{p}_{i}\right|_{\widehat{\mathrm{M}}}$ is a monomorphism.

Lemma 3.3. Let $i \in I$ and $\left(\widehat{\mathrm{M}}, z_{\widehat{\mathrm{M}}}\right)$ an affinization of a simple $R(\beta)$-module $M$. We set $m:=\varepsilon_{i}(M)$ and $m^{\prime}:=\varepsilon_{i}^{*}(M)$. Then we have

(i) $E_{i}^{m+1} \widehat{\mathrm{M}} \simeq 0$ and $E_{i}^{(m)} \widehat{\mathrm{M}}$ is an affinization of $E_{i}^{(m)} M$,

(ii) $E_{i}^{* m^{\prime}+1} \widehat{\mathrm{M}} \simeq 0$ and $E_{i}^{*\left(m^{\prime}\right)} \widehat{\mathrm{M}}$ is an affinization of $E_{i}^{*\left(m^{\prime}\right)} M$.

Proof. We shall only prove (i) since the case (ii) is similar. In the sequel, we ignore grading shifts. Let $d=\operatorname{deg} z_{\widehat{\mathrm{M}}}$. Applying the exact functor $E_{i}^{m+1}$ to

$$
0 \longrightarrow \widehat{\mathrm{M}} \stackrel{z_{\widehat{\mathrm{M}}}}{\longrightarrow} \widehat{\mathrm{M}} \longrightarrow M \longrightarrow 0,
$$


we have an exact sequence

$$
0 \longrightarrow E_{i}^{m+1} \widehat{\mathrm{M}} \stackrel{z}{\longrightarrow} E_{i}^{m+1} \widehat{\mathrm{M}} \longrightarrow 0 .
$$

Hence Nakayama's lemma shows $E_{i}^{m+1} \widehat{M} \simeq 0$. Applying the exact functor $E_{i}^{(m)}$ to the same exact sequence, we have an exact sequence

$$
0 \longrightarrow q^{d} \widehat{\mathrm{M}}_{0} \stackrel{\mathrm{z}}{\longrightarrow} \widehat{\mathrm{M}}_{0} \longrightarrow M_{0} \longrightarrow 0
$$

where $\widehat{\mathrm{M}}_{0}=E_{i}^{(m)} \widehat{\mathrm{M}}, M_{0}=E_{i}^{(m)} M$ and $\mathrm{z}=E_{i}^{(m)}\left(z_{\widehat{\mathrm{M}}}\right)$.

Thus, it remains to show that $\left.\mathfrak{p}_{j, \gamma}\right|_{\widehat{M}_{0}}$ does not vanish for any $j \in I$, where $\gamma=$ $\beta-m \alpha_{i}$. We can regard $\widehat{M}_{0}$ as a subspace of $\widehat{M}$ by

$$
\widehat{\mathrm{M}}_{0} \simeq\left\{u \in e\left(m \alpha_{i}, \beta-m \alpha_{i}\right) \widehat{\mathrm{M}} \mid \tau_{k} u=0 \text { for } 1 \leq k<m\right\}
$$

If $j \neq i$, then it is clear because $\left.\mathfrak{p}_{j, \gamma}\right|_{\widehat{M}_{0}}=\left.\mathfrak{p}_{j, \beta}\right|_{\widehat{M}_{0}}$ is injective. Suppose that $j=i$. Then, $\left.\mathfrak{p}_{i, \beta}\right|_{\widehat{M}_{0}}=\left.\left(\left(x_{1} \cdots x_{m}\right) \mathfrak{p}_{i, \gamma}\right)\right|_{\widehat{M}_{0}}$ is injective. Hence $\left.\mathfrak{p}_{i, \gamma}\right|_{\widehat{M}_{0}}$ is also injective.

In the sequel,

$$
t_{i} \text { is an indeterminate of degree }\left(\alpha_{i}, \alpha_{i}\right) \text {. }
$$

Definition 3.4. Let $i \in I$ and let $M$ be an $R(\beta)$-module. We set $m=\varepsilon_{i}(M), m^{\prime}=$ $\varepsilon_{i}^{*}(M)$ and $n=\mathrm{ht}(\beta)$. We define

$$
\begin{aligned}
\chi_{i}(M)\left(t_{i}\right) & :=\left.\left(\sum_{\nu \in I^{\beta}} \prod_{\nu_{k}=i}\left(t_{i}-x_{k}\right) e(\nu)\right)\right|_{M} \in \operatorname{END}_{R}(M)\left[t_{i}\right], \\
\mathfrak{e}_{i}(M)\left(t_{i}\right) & :=\left.\left(t_{i}-x_{1}\right) \cdots\left(t_{i}-x_{m}\right)\right|_{E_{i}^{(m)} M} \in \operatorname{END}_{R}\left(E_{i}^{(m)} M\right)\left[t_{i}\right], \\
\mathfrak{e}_{i}^{*}(M)\left(t_{i}\right) & :=\left.\left(t_{i}-x_{n-m^{\prime}+1}\right) \cdots\left(t_{i}-x_{n}\right)\right|_{E_{i}^{*\left(m^{\prime}\right)} M} \in \operatorname{END}_{R}\left(E_{i}^{*\left(m^{\prime}\right)} M\right)\left[t_{i}\right] .
\end{aligned}
$$

Note that $\chi_{i}(M), \mathfrak{e}_{i}(M)$ and $\mathfrak{e}_{i}^{*}(M)$ are homogeneous. If $M$ is a simple module, then $\chi_{i}(M), \mathfrak{e}_{i}(M)$ and $\mathfrak{e}_{i}^{*}(M)$ are powers of $t_{i}$. For an affinization $\widehat{\mathrm{M}}$ of a simple module, by Lemma 3.2 and Lemma 3.3, we have

$$
\chi_{i}(\widehat{\mathrm{M}}), \mathfrak{e}_{i}(\widehat{\mathrm{M}}), \mathfrak{e}_{i}^{*}(\widehat{\mathrm{M}}) \in \mathbf{k}\left[z_{\widehat{\mathrm{M}}}, t_{i}\right] .
$$

Lemma 3.5. Let $\gamma \in Q_{+}, m \in \mathbb{Z}_{>0}$ and $\beta=\gamma+m \alpha_{i}$. Let $M$ be a non-zero $R(\beta)$ module such that $\varepsilon_{i}(M)=m$. Set $B:=\operatorname{END}_{R}\left(E_{i}^{(m)} M\right)$. Let $f\left(t_{i}\right) \in B\left[t_{i}\right]$. Then the following conditions are equivalent:

(a) $f\left(x_{k}\right)=0$ in $\operatorname{END}_{\mathbf{k}}\left(E_{i}^{m} M\right)$ for any $k$ such that $1 \leq k \leq m$,

(b) $f\left(x_{k}\right)=0$ in $\operatorname{END}_{\mathbf{k}}\left(E_{i}^{m} M\right)$ for some $k$ such that $1 \leq k \leq m$,

(c) $\mathfrak{e}_{i}(M)\left(t_{i}\right)$ divides $f\left(t_{i}\right)$, i.e., $f\left(t_{i}\right) \in B\left[t_{i}\right] \mathfrak{e}_{i}(M)\left(t_{i}\right)$. 
If we assume further that $M$ is generated by $e\left(m \alpha_{i}, \gamma\right) M=E_{i}^{m} M$ as an $R(\beta)$-module and $f\left(t_{i}\right) \in \operatorname{End}_{R}(M)\left[t_{i}\right]$, then the equivalent conditions above implies (d) $\left.f\left(x_{1}\right)\right|_{E_{i} M}=0$.

Proof. Set $K=\left\{a(x) \in \operatorname{END}_{R}\left(E_{i}^{(m)} M\right)\left[x_{1}, \ldots, x_{m}\right] \mid a(x)\right.$ kills $\left.E_{i}^{m} M\right\}$. Then $K$ is stable by $s_{k}$ since $\varphi_{k} \cdot a(x) \cdot \varphi_{k}=s_{k}(a(x))$ (see Lemma 3.10 below). It is stable also by $\partial_{k}$ by (1.1). Hence the equivalence of (a) and (b) is obvious. Assuming that $f\left(x_{1}\right) \in K$, let us prove $f\left(t_{i}\right) \in B\left[t_{i}\right] \mathfrak{e}_{i}(M)$.

It is easy to see $\mathfrak{e}_{i}(M)\left(x_{1}\right) \in K$. By dividing $f\left(t_{i}\right)$ by the monic polynomial $\mathfrak{e}_{i}(M)\left(t_{i}\right)$ of degree $m$, we may assume that $f\left(t_{i}\right)$ is a polynomial in $t_{i}$ of degree strictly less than $m$. Write $f\left(t_{i}\right)=\sum_{j=0}^{n} a_{j} t_{i}^{j}$ with $0 \leq n<m$ and $a_{j} \in B$. In order to see $f\left(t_{i}\right)=0$, it is enough to show that $a_{n}=0$. We have $\partial_{n} \cdots \partial_{1} f\left(x_{1}\right)=(-1)^{n} a_{n} \in K$. Hence $a_{n}=0$.

Let us show (d). Set

$$
e=\sum_{\nu \in I^{\gamma}, \nu_{1} \neq i} e(\nu) \in R(\gamma)
$$

Then we have $e\left(m \alpha_{i}, \gamma\right) M=\left(e\left(m \alpha_{i}\right) \otimes e\right) M$. By the shuffle lemma, we have

$$
R(\beta) e\left(m \alpha_{i}, \gamma\right)=\sum_{w \in \mathfrak{S}_{m, n}} \tau_{w}\left(R\left(m \alpha_{i}\right) \otimes R(\gamma)\right)
$$

Here $\mathfrak{S}_{m, n}=\left\{w \in \mathfrak{S}_{m+n} \mid w(k)<w(k+1)\right.$ if $1 \leq k<m+n$ and $\left.k \neq m\right\}$. Hence $e\left(\alpha_{i}, \beta-\alpha_{i}\right) R(\beta)\left(e\left(m \alpha_{i}\right) \otimes e\right)=\sum_{w} \tau_{w} R\left(m \alpha_{i}\right) \otimes R(\gamma) e$, where $w$ ranges over $\mathfrak{S}_{m, n}$ and $w(m+1) \neq 1$. It implies $w(1)=1$. Hence we obtain

$$
e\left(\alpha_{i}, \beta-\alpha_{i}\right) R(\beta)\left(e\left(m \alpha_{i}\right) \otimes e\right) \subset\left(R\left(\alpha_{i}\right) \otimes R\left(\beta-\alpha_{i}\right)\right) \cdot\left(R\left(m \alpha_{i}\right) \otimes R(\gamma)\right) .
$$

Since we have a surjective map $P\left(i^{m}\right) \circ E_{i}^{(m)} M \rightarrow M$ by the assumption, we have

$$
\begin{aligned}
e\left(\alpha_{i}, \beta-\alpha_{i}\right)\left(P\left(i^{m}\right) \circ E_{i}^{(m)} M\right) & =e\left(\alpha_{i}, \beta-\alpha_{i}\right) R(\beta)\left(e\left(m \alpha_{i}\right) \otimes e\right)\left(P\left(i^{m}\right) \otimes E_{i}^{(m)} M\right) \\
& \subset\left(R\left(\alpha_{i}\right) \otimes R\left(\beta-\alpha_{i}\right)\right)\left(P\left(i^{m}\right) \otimes E_{i}^{(m)} M\right) .
\end{aligned}
$$

Hence we obtain

$$
e\left(\alpha_{i}, \beta-\alpha_{i}\right) M \subset\left(R\left(\alpha_{i}\right) \otimes R\left(\beta-\alpha_{i}\right)\right) e\left(m \alpha_{i}, \gamma\right) M .
$$

Thus $f\left(x_{1}\right)$ kills $e\left(\alpha_{i}, \beta-\alpha_{i}\right) M$.

Let $z$ be an indeterminate of degree $d \in \mathbb{Z}_{>0}$ and we set

$$
A:=\mathbf{k}[z] \text {. }
$$

Proposition 3.6. Let $i \in I$ and $m \in \mathbb{Z}_{>0}$. We take a homogeneous monic polynomial $f\left(t_{i}\right) \in A\left[t_{i}\right]$ such that 
(i) $\operatorname{deg}_{t_{i}} f\left(t_{i}\right)=m$,

(ii) $f(0) \neq 0$.

Then, there is an affinization $\widehat{\mathrm{M}}$ of $L\left(i^{m}\right)$ with $\chi_{i}(\widehat{\mathrm{M}})=f\left(t_{i}\right)$.

Proof. Let $R_{A}$ be a quiver Hecke algebra over the polynomial $\operatorname{ring} A$. We set $\Lambda:=m \Lambda_{i}$ and $a_{\Lambda}\left(t_{i}\right):=f\left(t_{i}\right)$. We now define

$$
\widehat{\mathrm{M}}:=F_{i}^{a_{\Lambda}(m)}(A) \in R_{A}^{a_{\Lambda}}\left(\Lambda-m \alpha_{i}\right)-\mathrm{Mod}
$$

and consider the endomorphism $z \in \operatorname{END}_{R}(\widehat{\mathrm{M}})$ defined by $u \mapsto z u$ for $u \in \widehat{\mathrm{M}}$. We shall prove that $(\widehat{\mathrm{M}}, z)$ is an affinization of $L\left(i^{m}\right)$.

Since the functor $F_{i}^{a_{\Lambda}(m)}$ gives an equivalence of the category of modules over $R_{A}^{a_{\Lambda}}(\Lambda-$ $\left.m \alpha_{i}\right)$ and the one over $R_{A}^{a_{\Lambda}}(\Lambda)=A$ by Proposition 1.4, we have

(i) $\widehat{\mathrm{M}}$ is a finitely generated projective module over $R_{A}^{a_{\Lambda}}\left(\Lambda-m \alpha_{i}\right)$.

(ii) $\operatorname{END}_{R_{A}^{a_{\Lambda}}\left(\Lambda-m \alpha_{i}\right)}(\widehat{\mathrm{M}}) \simeq A$.

(iii) the exact sequence $0 \rightarrow q^{2} A \stackrel{z}{\rightarrow} A \rightarrow \mathbf{k} \rightarrow 0$ gives the exact sequence

$$
0 \rightarrow q^{2} \widehat{\mathrm{M}} \stackrel{z}{\rightarrow} \widehat{\mathrm{M}} \rightarrow L\left(i^{m}\right) \rightarrow 0
$$

By (i), $\widehat{\mathrm{M}}$ is a finitely generated free module over $A$.

On the other hand, (ii) implies $\chi_{i}(\widehat{\mathrm{M}})\left(t_{i}\right) \in A\left[t_{i}\right]$ and we know that

(a) $\operatorname{deg}_{t_{i}}\left(f\left(t_{i}\right)\right)=m=\operatorname{deg}_{t_{i}}\left(\chi_{i}(\widehat{\mathrm{M}})\left(t_{i}\right)\right)$,

(b) $\left.f\left(x_{m}\right)\right|_{\widehat{M}}=\left.a_{\Lambda}\left(x_{m}\right)\right|_{\widehat{M}}=0$,

(c) $f\left(t_{i}\right)$ and $\chi_{i}(\widehat{\mathrm{M}})\left(t_{i}\right)$ are monic.

Thus, Lemma 3.5 implies that

$$
\chi_{i}(\widehat{\mathrm{M}})\left(t_{i}\right)=f\left(t_{i}\right)
$$

which tells, by the assumption,

$$
\left.\mathfrak{p}_{i}\right|_{\widehat{M}}=(-1)^{m} \chi_{i}(\widehat{M})(0)=(-1)^{m} f(0) \neq 0
$$

Therefore, $(\widehat{\mathrm{M}}, z)$ is an affinization of $L\left(i^{m}\right)$ with $\chi_{i}(\widehat{\mathrm{M}})=f\left(t_{i}\right)$.

Definition 3.7. For a homogeneous monic polynomial $f\left(t_{i}\right) \in A\left[t_{i}\right]$ such that

(i) $\operatorname{deg}_{t_{i}} f\left(t_{i}\right)=m$,

(ii) $f(0) \neq 0$,

we denote by $\mathrm{W}\left(i, f\left(t_{i}\right)\right)$ the affinization of $L\left(i^{m}\right)$ defined in Proposition 3.6. 
Remark 3.8. Let $\mathbf{k}\left[x_{1}, \ldots, x_{m}\right]$ be the faithful polynomial representation of $R\left(m \alpha_{i}\right)$ [18, Example 2.2]. Since $P\left(i^{m}\right)$ is isomorphic to $\mathbf{k}\left[x_{1}, \ldots, x_{m}\right]$ up to a grading shift, one can show that

$$
\mathrm{W}\left(i, f\left(t_{i}\right)\right) \simeq \mathbf{k}\left[z, x_{1}, \ldots, x_{m}\right] /\left(\sum_{j=1}^{m} \mathbf{k}\left[z, x_{1}, \ldots, x_{m}\right]\left(e_{j}(x)-a_{j}(z)\right)\right)
$$

up to a grading shift. Here $e_{j}(x)(1 \leq j \leq m)$ is the $j$-the elementary symmetric polynomial in $\left(x_{1}, \ldots, x_{m}\right)$ and $f\left(t_{i}\right)=\sum_{j=0}^{m}(-1)^{j} a_{j}(z) t_{i}^{m-j}\left(a_{j}(z) \in \mathbf{k}[z]\right)$.

Lemma 3.9. Let $A$ be a commutative $\mathbf{k}$-algebra, $i \in I$ and let $\widehat{\mathrm{M}}$ be an $R_{A}(\beta)$-module. We set $m:=\varepsilon_{i}(\widehat{\mathrm{M}})$. Let $h: \operatorname{END}_{R_{A}}(\widehat{\mathrm{M}}) \rightarrow \operatorname{END}_{R_{A}}\left(E_{i}^{(m)} \widehat{\mathrm{M}}\right)$ be the canonical map. Then we have $h\left(\chi_{i}(\widehat{\mathrm{M}})\right)=\mathfrak{e}_{i}(\widehat{\mathrm{M}}) \chi_{i}\left(E_{i}^{(m)} \widehat{\mathrm{M}}\right)$ as elements of $\operatorname{END}_{R_{A}}\left(E_{i}^{(m)} \widehat{\mathrm{M}}\right)\left[t_{i}\right]$.

Similarly, setting $m^{\prime}:=\varepsilon_{i}^{*}(M)$ and $h^{*}: \operatorname{END}_{R_{A}}(\widehat{\mathrm{M}}) \rightarrow \operatorname{END}_{R_{A}}\left(E_{i}^{*\left(m^{\prime}\right)} \widehat{\mathrm{M}}\right)$ the canonical map, we have $h^{*}\left(\chi_{i}(\widehat{\mathrm{M}})\right)=\chi_{i}\left(E_{i}^{*}\left(m^{\prime}\right) \widehat{M}\right) \mathfrak{e}_{i}^{*}(\widehat{\mathrm{M}})$.

Proof. Set $\gamma=\beta-m \alpha_{i}$ and $\operatorname{ch}_{i, \beta}\left(t_{i}\right)=\sum_{\nu \in I^{\beta}} \prod_{\nu_{k}=i}\left(t_{i}-x_{k}\right) e(\nu) \in R(\beta)\left[t_{i}\right]$. Then we have $\operatorname{ch}_{i, \beta}\left(t_{i}\right) e\left(m \alpha_{i}, \gamma\right)=\operatorname{ch}_{i, m \alpha_{i}}\left(t_{i}\right) \otimes \operatorname{ch}_{i, \gamma}\left(t_{i}\right)$. Hence we have

$$
\begin{aligned}
\left.\operatorname{ch}_{i, \beta}\left(t_{i}\right)\right|_{E_{i}^{(m)} \widehat{M}} & =\left.\left.\left(\prod_{k=1}^{m}\left(t_{i}-x_{k}\right)\right)\right|_{E_{i}^{(m)} \widehat{M}} \cdot \operatorname{ch}_{i, \gamma}\left(t_{i}\right)\right|_{E_{i}^{(m)} \widehat{M}} \\
& =\mathfrak{e}_{i}(\widehat{\mathrm{M}}) \chi_{i}\left(E_{i}^{(m)} \widehat{\mathrm{M}}\right) .
\end{aligned}
$$

The case for $E_{i}^{*}$ can be proved similarly.

3.2. R-matrices. Let $\beta \in Q_{+}$with $m=\operatorname{ht}(\beta)$. For $k=1, \ldots, m-1$ and $\nu \in I^{\beta}$, the intertwiner $\varphi_{k}$ is defined by

$$
\varphi_{k} e(\nu)= \begin{cases}\left(\tau_{k} x_{k}-x_{k} \tau_{k}\right) e(\nu)=\left(x_{k+1} \tau_{k}-\tau_{k} x_{k+1}\right) e(\nu) & \text { if } \nu_{k}=\nu_{k+1} \\ \tau_{k} e(\nu) & \text { otherwise }\end{cases}
$$

Lemma 3.10 ([12, Lemma 1.5]).

(i) $\varphi_{k}^{2} e(\nu)=\left(Q_{\nu_{k}, \nu_{k+1}}\left(x_{k}, x_{k+1}\right)+\delta_{\nu_{k}, \nu_{k+1}}\right) e(\nu)$.

(ii) $\left\{\varphi_{k}\right\}_{k=1, \ldots, m-1}$ satisfies the braid relation.

(iii) For a reduced expression $w=s_{i_{1}} \cdots s_{i_{t}} \in \mathfrak{S}_{m}$, we set $\varphi_{w}:=\varphi_{i_{1}} \cdots \varphi_{i_{t}}$. Then $\varphi_{w}$ does not depend on the choice of reduced expression of $w$.

(iv) For $w \in \mathfrak{S}_{m}$ and $1 \leq k \leq m$, we have $\varphi_{w} x_{k}=x_{w(k)} \varphi_{w}$.

(v) For $w \in \mathfrak{S}_{m}$ and $1 \leq k<m$, if $w(k+1)=w(k)+1$, then $\varphi_{w} \tau_{k}=\tau_{w(k)} \varphi_{w}$. 
For $m, n \in \mathbb{Z}_{\geq 0}$, we set $w[m, n]$ to be the element of $\mathfrak{S}_{m+n}$ such that

$$
w[m, n](k):= \begin{cases}k+n & \text { if } 1 \leq k \leq m, \\ k-m & \text { if } m<k \leq m+n .\end{cases}
$$

Let $\beta, \gamma \in Q_{+}$and set $m:=\operatorname{ht}(\beta)$ and $n:=\operatorname{ht}(\gamma)$. For $M \in R(\beta)$-Mod and $N \in$ $R(\gamma)$-Mod, the $R(\beta) \otimes R(\gamma)$-linear map $M \otimes N \rightarrow N \circ M$ defined by $u \otimes v \mapsto \varphi_{w[n, m]}(v \otimes$ u) can be extended to an $R(\beta+\gamma)$-module homomorphism (up to a grading shift)

$$
\mathrm{R}_{M, N}: M \circ N \longrightarrow N \circ M \text {. }
$$

Let $\widehat{\mathrm{M}}$ be an affinization of a simple $R$-module $M$, and let $N$ be a non-zero $R$-module. We define a homomorphism (up to a grading shift)

$$
\mathrm{R}_{\widehat{\mathrm{M}}, N}^{\text {norm }}:=z_{\widehat{\mathrm{M}}}^{-s} \mathrm{R}_{\widehat{\mathrm{M}}, N}: \widehat{\mathrm{M}} \circ N \longrightarrow N \circ \widehat{\mathrm{M}}
$$

where $s$ is the largest integer such that $\mathrm{R}_{\widehat{\mathrm{M}}, N}(\widehat{\mathrm{M}} \circ N) \subset z_{\widehat{\mathrm{M}}} s(N \circ \widehat{\mathrm{M}})$. We define

$$
\mathbf{r}_{M, N}: M \circ N \longrightarrow N \circ M
$$

to be the homomorphism (up to a grading shift) induced from $\mathrm{R}_{\widehat{\mathrm{M}}, N}^{\text {norm }}$ by specializing at $z_{\widehat{\mathrm{M}}}=0$. By the definition, $\mathbf{r}_{M, N}$ never vanishes. We now define

$$
\begin{aligned}
\Lambda(M, N) & :=\operatorname{deg}\left(\mathbf{r}_{M, N}\right), \\
\widetilde{\Lambda}(M, N) & :=\frac{1}{2}(\Lambda(M, N)+(\operatorname{wt}(M), \operatorname{wt}(N))), \\
\mathfrak{D}(M, N) & :=\frac{1}{2}(\Lambda(M, N)+\Lambda(N, M)) .
\end{aligned}
$$

Lemma 3.11. Let $M$ be a simple $R(\alpha)$-module and $N$ a simple $R(\beta)$-module. Assume that one of them is real simple and admits an affinization of degree $d$. Then one has

$$
\widetilde{\Lambda}(M, N), \quad \mathfrak{D}(M, N) \in \frac{d}{2} \mathbb{Z}_{\geq 0}
$$

Proof. We treat only the case when $M$ is real and admits an affinization $\left(\widehat{\mathrm{M}}, z_{\widehat{\mathrm{M}}}\right)$.

Set $m=\operatorname{ht}(\alpha)$ and $n=\operatorname{ht}(\beta)$. For $w \in \mathfrak{S}_{r}$, choosing a reduced expression $s_{i_{1}} \cdots s_{i_{\ell}}$ of $w$, we write $\tau_{w}=\tau_{i_{1}} \cdots \tau_{i_{\ell}}$.

Then, by $[12,(1.17)]$, we have for $u \in \widehat{M}$ and $v \in N$

$$
\mathrm{R}_{\widehat{\mathrm{M}}, N}(u \otimes v) \in \tau_{w[n, m]}\left(\sum_{\mu \in I^{\alpha}, \nu \in I^{\beta}} \prod_{(i, j) \in B(\nu, \mu)}\left(x_{i}-x_{j}\right) e(\nu, \mu)\right) v \nabla u+\sum_{w \prec w[n, m]} \tau_{w}(N \otimes \widehat{\mathrm{M}}),
$$


where $B(\nu, \mu)=\left\{(i, j) \mid 1 \leq i \leq n<j \leq m+n, \nu_{i}=\mu_{j}\right\}$. We have

$$
\begin{aligned}
\left(\sum_{\mu \in I^{\alpha}, \nu \in I^{\beta}} \prod_{(i, j) \in B(\nu, \mu)}\left(x_{i}-x_{j}\right) e(\nu, \mu)\right) v \otimes u & =\left(\sum_{\mu \in I^{\alpha}, \nu \in I^{\beta}} \prod_{(i, j) \in B(\nu, \mu)}\left(-x_{j}\right) e(\nu, \mu)\right) v \otimes u \\
& = \pm v \otimes\left(\prod_{i \in I} \mathfrak{p}_{i, \alpha}\right)^{\frac{2\left(\beta, \Lambda_{i}\right)}{\left(\alpha_{i}, \alpha_{i}\right)}} u=c z_{\widehat{\mathrm{M}}}{ }^{s} u
\end{aligned}
$$

for some $c \in \mathbf{k}^{\times}$and $s \in \mathbb{Z}_{\geq 0}$. Therefore, we obtain

$$
\mathrm{R}_{\widehat{\mathrm{M}}, N}(u \otimes v) \in c z_{\widehat{\mathrm{M}}}^{s} \tau_{w[n, m]} e(\beta, \alpha)(v \otimes u)+\sum_{w \prec w[n, m]} \tau_{w}(N \otimes \widehat{\mathrm{M}}) .
$$

Thus we conclude

$$
\mathrm{R}_{\widehat{\mathrm{M}}, N}^{\mathrm{norm}}(u \otimes v) \in c z_{\widehat{\mathrm{M}}}^{a} \tau_{w[n, m]} e(\beta, \alpha)(v \nabla u)+\sum_{w \prec w[n, m]} \tau_{w}(N \otimes \widehat{\mathrm{M}})
$$

for some $a \in \mathbb{Z}_{\geq 0}$. Then we obtain

$$
\begin{aligned}
2 \widetilde{\Lambda}(M, N) & =\operatorname{deg}\left(\mathrm{R}_{\widehat{\mathrm{M}}, N}^{\text {norm }}\right)+(\alpha, \beta) \\
& =\operatorname{deg}\left(z_{\widehat{\mathrm{M}}}{ }^{a} \tau_{w[n, m]} e(\beta, \alpha)\right)+(\alpha, \beta) \\
& =\operatorname{deg}\left(z_{\widehat{\mathrm{M}}}{ }^{a}\right) \in d \mathbb{Z}_{\geq 0} .
\end{aligned}
$$

Now let us show the case of $\mathfrak{d}(M, N)$. By Proposition 3.13 (iii) below, we have

$$
\mathrm{R}_{N, \widehat{M}}^{\text {norm }} \circ \mathrm{R}_{\widehat{\mathrm{M}}, N}^{\text {norm }}=c z_{\widehat{\mathrm{M}}} s \mathrm{id}_{\widehat{\mathrm{M}} \circ N}
$$

for some $c \in \mathbf{k}^{\times}$and $s \in \mathbb{Z}_{\geq 0}$. Then we have

$$
\begin{aligned}
2 \mathrm{D}(M, N) & =\operatorname{deg}\left(\mathrm{R}_{N, \widehat{\mathrm{M}}}^{\text {norm }} \circ \mathrm{R}_{\widehat{\mathrm{M}}, N}^{\text {norm }}\right) \\
& =\operatorname{deg}\left(z_{\widehat{\mathrm{M}}}^{s}\right) \in d \mathbb{Z}_{\geq 0} .
\end{aligned}
$$

Corollary 3.12. Let $M$ be a simple module. We assume that $\mathrm{wt}(M) \neq 0$ and $M$ is real and admits an affinization. Then we have

$$
(\operatorname{wt}(M), \operatorname{wt}(M))>0 .
$$

Proof. Since $\Lambda(M, M)=0$, we have

$$
0 \leq 2 \widetilde{\Lambda}(M, M)=(\mathrm{wt}(M), \mathrm{wt}(M)) .
$$

If $(\operatorname{wt}(M), \operatorname{wt}(M))=0$, then $\widetilde{\Lambda}(M, M)=0$ and one has

$$
\mathbf{r}_{M, M}(u \otimes v) \in \tau_{w[n, n]}(v \otimes u)+\sum_{w<w[n, n]} \tau_{w}(M \otimes M) .
$$


Hence $\mathbf{r}_{M, M} \notin \mathbf{k}$ id $_{M \circ M}$, which contradicts the fact that $M \circ M$ is simple.

Proposition 3.13 ([16, Proposition 2.10, Proposition 2.11]). Let $M$ and $N$ be simple $R$-modules. Suppose that $M$ is real and admits an affinization $\widehat{\mathrm{M}}$.

(i) $M \circ N$ has a simple head and a simple socle. Moreover, $\operatorname{Im}\left(\mathbf{r}_{M, N}\right)$ is equal to $M \nabla N$ and $N \Delta M$ up to grading shifts.

$$
\begin{array}{ll}
\operatorname{Hom}_{R}(M \circ N, M \circ N)=\mathbf{k} \text { id }_{M \circ N}, & \operatorname{Hom}_{R}(N \circ M, N \circ M)=\mathbf{k} \text { id }_{N \circ M}, \\
\operatorname{Hom}_{R}(M \circ N, N \circ M)=\mathbf{k} \mathbf{r}_{M, N}, & \operatorname{Hom}_{R}(N \circ M, M \circ N)=\mathbf{k} \mathbf{r}_{N, M} .
\end{array}
$$

(iii)

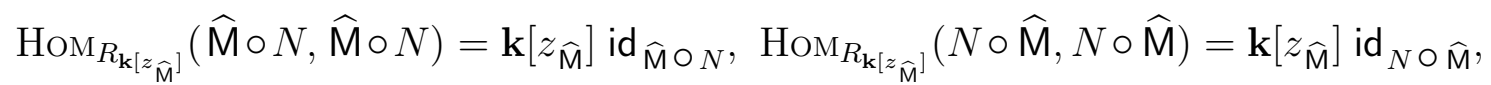

$$
\begin{aligned}
& \operatorname{Hom}_{R_{\mathbf{k}\left[z_{\widehat{M}}\right]}}(\widehat{M} \circ N, N \circ \widehat{M})=\mathbf{k}\left[z_{\widehat{M}}\right] R_{\widehat{M}, N}^{\text {norm }}, \quad \operatorname{Hom}_{R_{\mathbf{k}\left[z_{\widehat{M}}\right]}}(N \circ \widehat{M}, \widehat{M} \circ N)=\mathbf{k}\left[z_{\widehat{M}}\right] R_{N, \widehat{M}}^{\text {norm }} .
\end{aligned}
$$

Now assume that $N$ has an affinization $\left(\widehat{N}, z_{\widehat{N}}\right)$. Then similarly to the case of $M$, we can define $\mathrm{R}_{M, \widehat{\mathrm{N}}}^{\text {norm }}$. The proposition above implies that $\operatorname{deg} \mathrm{R}_{\widehat{\mathrm{M}}, N}^{\text {norm }}=\operatorname{deg} \mathrm{R}_{M, \widehat{N}}^{\text {norm }}$, and $\left.\mathrm{R}_{\widehat{\mathrm{M}}, N}^{\text {norm }}\right|_{z_{\widehat{\mathrm{M}}}=0}=\left.\mathrm{R}_{M, \widehat{\mathrm{N}}}^{\text {norm }}\right|_{z_{\widehat{\mathrm{N}}}=0}$ up to a constant multiple. Hence $\Lambda(M, N)$ and $\mathbf{r}_{M, N}$ (up to a constant multiple) are well defined when either $M$ or $N$ admits an affinization. Moreover, they do not depend on the choice of the affinization.

Lemma $3.14-3.21$ below were proved when $R$ is symmetric. However they still hold also for non-symmetric case. Since the proofs are similar, we omit the proofs.

Lemma 3.14 ([14, Lemma 3.1.4]). Let $M$ and $N$ be self-dual simple $R$-modules. If one of them is real and also admits an affinization, then $q^{\widetilde{\Lambda}(M, N)} M \nabla N$ is a self-dual simple R-module.

Lemma 3.15 (cf. [15, Corollary 3.8]). Let $M$ be a simple R-module. For $i \in I$, we have

$$
\begin{aligned}
& \Lambda(L(i), M)=\left(\alpha_{i}, \alpha_{i}\right) \varepsilon_{i}(M)+\left(\alpha_{i}, \operatorname{wt}(M)\right), \\
& \Lambda(M, L(i))=\left(\alpha_{i}, \alpha_{i}\right) \varepsilon_{i}^{*}(M)+\left(\alpha_{i}, \operatorname{wt}(M)\right) .
\end{aligned}
$$

Lemma 3.16 ([14, Proposition 3.2.8 and 3.2.10]). Let $M, N, N^{\prime}$ and $L$ be simple modules. Assume that $M$ is real and admits an affinization. Let $N \circ N^{\prime} \rightarrow L$ be an epimorphism. Then we have

(i) $\Lambda(M, L) \leq \Lambda(M, N)+\Lambda\left(M, N^{\prime}\right)$, 
(ii) if $\Lambda(M, L)=\Lambda(M, N)+\Lambda\left(M, N^{\prime}\right)$, then the following diagram commutes up to a constant multiple.

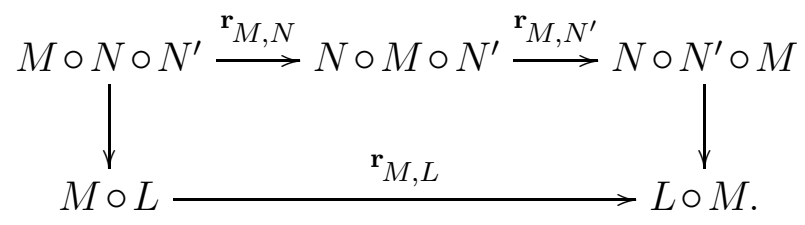

Note that $L \simeq N \nabla N^{\prime}$ if either $N$ or $N^{\prime}$ is real and admits an affinization.

Lemma 3.17 ([14, Corollary 4.1.2]). Let $M$ and $N$ be simple $R$-modules such that $M$ and $N$ do not strongly commute. Assume that either $M$ or $N$ is real and admits an affinization. Then, in the Grothendieck group $K(R$-gmod), we can write

$$
[M \circ N]=[M \nabla N]+[M \Delta N]+\sum_{k}\left[S_{k}\right]
$$

for some simple $R$-modules $S_{k}$.

(i) If $M$ is real and admits an affinization, then

$$
\begin{aligned}
& \Lambda(M, M \Delta N), \Lambda\left(M, S_{k}\right)<\Lambda(M, N)=\Lambda(M, M \nabla N), \\
& \Lambda(M \nabla N, M), \Lambda\left(S_{k}, M\right)<\Lambda(N, M)=\Lambda(N \nabla M, M) .
\end{aligned}
$$

In particular $\mathfrak{D}(M, S)<\mathfrak{D}(M, N)$ for any simple subquotient $S$ of $M \circ N$.

(ii) If $N$ is real and admits an affinization, then

$$
\begin{aligned}
& \Lambda(N, M \nabla N), \Lambda\left(N, S_{k}\right)<\Lambda(N, M)=\Lambda(N, N \nabla M) \\
& \Lambda(M \Delta N, N), \Lambda\left(S_{k}, N\right)<\Lambda(M, N)=\Lambda(M \nabla N, N) .
\end{aligned}
$$

In particular $\mathfrak{D}(S, N)<\mathfrak{D}(M, N)$ for any simple subquotient $S$ of $M \circ N$.

Corollary 3.18. Let $M$ be a real simple module which admits an affinization. Let $X$ be an $R$-module in $R$-gmod. Let $n \in \mathbb{Z}_{>0}$ and assume that any simple subquotient $S$ of $X$ satisfies $\mathrm{D}(M, S) \leq n$. Then any simple subquotient $S$ of $M \circ X$ satisfies $\mathrm{D}(M, S)<n$. In particular, any simple subquotient of $M^{\circ} \circ X$ strongly commutes with $M$.

Proof. We can reduce to the case where $X$ is simple. Then the assertion follows from Lemma 3.17.

Lemma 3.19 ([14, Theorem 4.1.3]). Let $M$ be a simple $R$-module, and let $x$ be the element of the Grothendieck group $K(R$-gmod) given by

$$
x=\sum_{b \in B(\infty)} a_{b}\left[L_{b}\right]
$$


where $L_{b}$ is the self-dual simple module corresponding to $b$ in the crystal $B(\infty)$ of $U_{q}^{-}(\mathfrak{g})$ and $a_{b} \in \mathbb{Z}\left[q, q^{-1}\right]$. If

(a) $M$ is real and admits an affinization,

(b) $x[M]=q^{l}[M] x$ for some $l \in \mathbb{Z}$,

then we have, for $b \in B(\infty)$ with $a_{b} \neq 0$,

(i) $M$ strongly commutes with $L_{b}$,

(ii) $\Lambda\left(M, L_{b}\right)=l$.

Lemma 3.20 ([14, Corollary 4.1.4]). Let $M$ and $N$ be simple R-modules. Suppose that

(i) either $M$ or $N$ is real and admits an affinization,

(ii) $[M][N]=q^{l}[N][M]$ for some $l \in \mathbb{Z}$ in the Grothendieck group $K(R$-gmod).

Then, $M$ and $N$ strongly commute and $\Lambda(M, N)=-\Lambda(N, M)=-l$.

Lemma 3.21 ([13, Proposition 3.6, Corollary 3.7]). Let $M$ be a simple $R(\beta)$-module. Suppose that $M$ is real and admits an affinization. Then we have the following.

(i) For a simple $R(\beta+\gamma)$-module $L$, the $R(\gamma)$-module $\operatorname{Hom}_{R(\beta+\gamma)}(M \circ R(\gamma), L)$ is either zero or has a simple socle.

(ii) The map $N \mapsto M \nabla N$ between the set of the isomorphism classes of simple $R$-modules is injective.

Proposition 3.22. Let $M, N$ and $N^{\prime}$ be simple $R$-modules. Suppose that $M$ is real and admits an affinization. If there exists non-zero homomorphism $f \in \operatorname{Hom}_{R}(M \circ N$, $\left.N^{\prime} \circ M\right)$, then $N \simeq N^{\prime}$ up to a grading shift.

Proof. Suppose that $N \not N^{\prime}$. Since $M$ is real, $M \nabla N$ is simple and appears in $\operatorname{Im}(f) \subset N^{\prime} \circ M$ as a subquotient, which tells that $M \nabla N$ also appears in $M \circ N^{\prime}$ as a subquotient. As $N \nsucceq N^{\prime}$, Lemma 3.21 implies $M \nabla N \not M \nabla N^{\prime}$. Thus, by Lemma 3.17, we have

$$
\Lambda(M, N)=\Lambda(M, M \nabla N)<\Lambda\left(M, N^{\prime}\right)
$$

On the other hand, taking the dual of $f$, we have a non-zero homomorphism $f^{\star}: M \circ N^{\prime} \rightarrow N \circ M$ up to a grading shift. Thus, by the same argument as above, we obtain

$$
\Lambda\left(M, N^{\prime}\right)<\Lambda(M, N)
$$

which is a contradiction. Therefore, $N$ and $N^{\prime}$ are isomorphic to each other. 


\subsection{Determinantial modules.}

Let $A=\oplus_{n \geq 0} A_{n}$ be a commutative positively graded algebra as in Section 1.3. We assume $A_{0}=\mathbf{k}$ for simplicity. Let $\Lambda \in \mathrm{P}_{+}$. We take a family $a_{\Lambda}=\left\{a_{\Lambda, i}\left(t_{i}\right)\right\}$ of parameters in Section 1.3 such that $a_{\Lambda, i}\left(t_{i}\right) \in A\left[t_{i}\right]$ is monic. Recall that $R_{A}^{a_{\Lambda}}(\lambda)$ denotes the associated cyclotomic quiver Hecke algebra for $\lambda \in \mathrm{P}$.

Let $\lambda, \mu \in \mathrm{W} \Lambda$ and we choose $w, v \in \mathrm{W}$ such that $\lambda=w \Lambda$ and $\mu=v \Lambda$. Take reduced expressions $\underline{w}=s_{i_{1}} \cdots s_{i_{l}}$ and $\underline{v}=s_{j_{1}} \cdots s_{j_{t}}$, and set $m_{k}=\left\langle h_{i_{k}}, s_{i_{k+1}} \cdots s_{i_{l}} \Lambda\right\rangle$ for $k=1, \ldots l$, and $n_{k}=\left\langle h_{j_{k}}, s_{j_{k+1}} \cdots s_{j_{t}} \Lambda\right\rangle$ for $k=1, \ldots, t$. We define

$$
\begin{aligned}
& \mathrm{M}\left(\lambda, \Lambda ; a_{\Lambda}\right):=F_{i_{1}}^{a_{\Lambda}\left(m_{1}\right)} \cdots F_{i_{l}}^{a_{\Lambda}\left(m_{l}\right)} A, \\
& \mathrm{M}\left(\lambda, \mu ; a_{\Lambda}\right):=E_{j_{1}}^{*\left(n_{1}\right)} \cdots E_{j_{t}}^{*\left(n_{t}\right)} \mathrm{M}\left(\lambda, \Lambda ; a_{\Lambda}\right),
\end{aligned}
$$

where $A$ is regarded as the module over $R_{A}^{\Lambda}(\Lambda) \simeq A$.

When $A=\mathbf{k}$ and $a_{\Lambda}=\left\{a_{\Lambda, i}\left(t_{i}\right)\right\}$ is given by $a_{\Lambda, i}\left(t_{i}\right)=t_{i}^{\left\langle h_{i}, \Lambda\right\rangle}$, we write $\mathrm{M}(\lambda, \Lambda)$ and $\mathrm{M}(\lambda, \mu)$ instead of $\mathrm{M}\left(\lambda, \Lambda ; a_{\Lambda}\right)$ and $\mathrm{M}\left(\lambda, \mu ; a_{\Lambda}\right)$.

Proposition 3.23 ([11] and [15, Lemma 1.7, Proposition 4.2]). Let $\Lambda \in \mathrm{P}_{+}$, and $\lambda, \mu \in \mathrm{W} \Lambda$ with $\lambda \preceq \mu$.

(i) $\mathrm{M}\left(\lambda, \mu ; a_{\Lambda}\right)$ is a finitely generated projective $A$-module.

(ii) $\mathrm{M}(\lambda, \mu)$ is a real simple module.

(iii) If $\left\langle h_{i}, \lambda\right\rangle \leq 0$ and $s_{i} \lambda \preceq \mu$, then

$$
\varepsilon_{i}\left(\mathrm{M}\left(\lambda, \mu ; a_{\Lambda}\right)\right)=-\left\langle h_{i}, \lambda\right\rangle \quad \text { and } \quad E_{i}^{\left(-\left\langle h_{i}, \lambda\right\rangle\right)} \mathrm{M}\left(\lambda, \mu ; a_{\Lambda}\right) \simeq \mathrm{M}\left(s_{i} \lambda, \mu ; a_{\Lambda}\right) .
$$

(iv) If $\left\langle h_{i}, \mu\right\rangle \geq 0$ and $\lambda \preceq s_{i} \mu$, then

$$
\varepsilon_{i}^{*}\left(\mathrm{M}\left(\lambda, \mu ; a_{\Lambda}\right)\right)=\left\langle h_{i}, \mu\right\rangle \quad \text { and } \quad E_{i}^{*\left(\left\langle h_{i}, \mu\right\rangle\right)} \mathrm{M}\left(\lambda, \mu ; a_{\Lambda}\right) \simeq \mathrm{M}\left(\lambda, s_{i} \mu ; a_{\Lambda}\right) .
$$

Proof. (i) follows from [11] and (ii)-(iv) for $\mathrm{M}(\lambda, \mu)$ follows from [15, Lemma 1.7, Proposition 4.2]. (iii) and (iv) for $\mathrm{M}\left(\lambda, \mu ; a_{\Lambda}\right)$ follows from the case $\mathrm{M}(\lambda, \mu)$ by noticing that $\mathbf{k} \otimes_{A} \mathrm{M}\left(\lambda, \mu ; a_{\Lambda}\right) \simeq \mathrm{M}(\lambda, \mu)$, and hence $\mathrm{M}\left(\lambda, \mu ; a_{\Lambda}\right) \simeq 0$ if and only if $\mathrm{M}(\lambda, \mu) \simeq 0$

Proposition 3.24 ([15, Corollary 4.7]). Let $\Lambda \in \mathrm{P}_{+}$.

(i) Let $i \in I$ and $v \in \mathrm{W}$ such that $v s_{i}\left\langle v\right.$ and $n:=\left\langle h_{i}, \Lambda\right\rangle>0$. Then $\mathrm{M}\left(v \Lambda, v s_{i} \Lambda\right)$ is a $\preceq$-cuspidal $R\left(-n v \alpha_{i}\right)$-module, where $\preceq$ is a convex order corresponding to a reduced expression of $v$ given in Proposition 1.8.

(ii) Let $\preceq$ be a convex order corresponding to a reduced expression $\underline{w}=s_{i_{1}} \cdots s_{i_{\ell}}$ of $w \in$ $\mathrm{W}$ given in Proposition 1.8. Then the sequence obtained from $\left(\mathrm{M}\left(w_{\ell} \Lambda, w_{\ell-1} \Lambda\right)\right.$, $\left.\ldots, \mathrm{M}\left(w_{1} \Lambda, w_{0} \Lambda\right)\right)$ by removing $\mathrm{M}\left(w_{k} \Lambda, w_{k-1} \Lambda\right)$ such that $w_{k} \Lambda=w_{k-1} \Lambda$, is a $\preceq$-cuspidal decomposition of $\mathrm{M}(w \Lambda, \Lambda)$, where $w_{k}=s_{i_{1}} \cdots s_{i_{k}}$ for $k=1, \ldots, \ell$. 
By Proposition 1.4, we have

$$
\operatorname{END}_{R_{A}^{a_{\Lambda}}}\left(\mathrm{M}\left(\lambda, \Lambda ; a_{\Lambda}\right)\right) \simeq \operatorname{END}_{R_{A}^{a_{\Lambda}}(\Lambda)}(A) \simeq A .
$$

Hence $\chi_{i}\left(\mathrm{M}\left(\lambda, \Lambda ; a_{\Lambda}\right)\right)$ and $\mathfrak{e}_{i}\left(\mathrm{M}\left(\lambda, \Lambda ; a_{\Lambda}\right)\right)$ are regarded as elements of $A\left[t_{i}\right]$. Note that, by Lemma 3.9 and $(3.3)$, if $\left\langle h_{i}, \lambda\right\rangle \leq 0$, we have

$$
\chi_{i}\left(\mathrm{M}\left(\lambda, \Lambda ; a_{\Lambda}\right)\right)=\mathfrak{e}_{i}\left(\mathrm{M}\left(\lambda, \Lambda ; a_{\Lambda}\right)\right) \chi_{i}\left(\mathrm{M}\left(s_{i} \lambda, \Lambda ; a_{\Lambda}\right)\right) \in A\left[t_{i}\right] .
$$

For $i \in I$ and $\mu \in \mathrm{W} \Lambda$, we set

$$
F_{i, \mu}\left(t_{i}\right):=\left.a_{\Lambda, i}\left(t_{i}\right) \cdot\left(\sum_{\nu \in I^{\Lambda-\mu}} \prod_{1 \leq k \leq n, \nu_{k} \neq i} \mathcal{Q}_{i, \nu_{k}}\left(t_{i}, x_{k}\right) e(\nu)\right)\right|_{\mathrm{M}\left(\mu, \Lambda ; a_{\Lambda}\right)} \in A\left[t_{i}\right] .
$$

Here $n=\operatorname{ht}(\Lambda-\mu)$.

For $i, j \in I$ such that $i \neq j$ and $\mu \in \mathrm{W} \Lambda$ such that $n:=-\left\langle h_{j}, \mu\right\rangle \geq 0$, we set

$$
\mathrm{q}_{i, j, \mu}\left(t_{i}\right):=\left.\left(\prod_{k=1}^{n} \mathcal{Q}_{i, j}\left(t_{i}, x_{k}\right)\right)\right|_{e\left(j^{n}, *\right) \mathrm{M}\left(\mu, \Lambda ; a_{\Lambda}\right)} \in A\left[t_{i}\right] .
$$

Thanks to $(3.3), F_{i, \mu}\left(t_{i}\right)$ and $\mathrm{q}_{i, j, \mu}\left(t_{i}\right)$ can be viewed as quasi-monic polynomials in $A\left[t_{i}\right]$.

\section{Proposition 3.25.}

(i) For any $\mu \in \mathrm{W} \Lambda$, we have

$$
F_{i, \mu}\left(t_{i}\right)=\gamma \chi_{i}\left(\mathrm{M}\left(\mu, \Lambda ; a_{\Lambda}\right)\right)\left(t_{i}\right) \cdot \chi_{i}\left(\mathrm{M}\left(s_{i} \mu, \Lambda ; a_{\Lambda}\right)\right)\left(t_{i}\right)
$$

for some $\gamma \in \mathbf{k}^{\times}$.

(ii) For $i, j \in I$ and $\lambda \in \mathrm{W} \Lambda$ such that $\lambda \prec s_{i} \lambda \prec s_{j} s_{i} \lambda$, set $\mu=s_{i} \lambda$ and $\zeta=s_{j} \mu$. Then we have

$$
\mathfrak{e}_{i}\left(\mathrm{M}\left(\lambda, \Lambda ; a_{\Lambda}\right)\right)\left(t_{i}\right)= \begin{cases}\gamma \mathrm{q}_{i, j, \mu}\left(t_{i}\right) \mathfrak{e}_{i}\left(\mathrm{M}\left(s_{i} \zeta, \Lambda ; a_{\Lambda}\right)\right)\left(t_{i}\right) & \text { if }\left\langle h_{i}, \zeta\right\rangle \geq 0 \\ \frac{\gamma \mathrm{q}_{i, j, \mu}\left(t_{i}\right)}{\mathfrak{e}_{i}\left(\mathrm{M}\left(\zeta, \Lambda ; a_{\Lambda}\right)\right)\left(t_{i}\right)} & \text { if }\left\langle h_{i}, \zeta\right\rangle \leq 0\end{cases}
$$

for some $\gamma \in \mathbf{k}^{\times}$.

Proof. For simplicity, we set

$$
\mathrm{M}(\xi):=\mathrm{M}\left(\xi, \Lambda ; a_{\Lambda}\right) \quad \text { and } \quad \chi_{i, \xi}\left(t_{i}\right):=\chi_{i}(\mathrm{M}(\xi))\left(t_{i}\right), \quad \mathfrak{e}_{i, \xi}\left(t_{i}\right):=\mathfrak{e}_{i}(\mathrm{M}(\xi))\left(t_{i}\right),
$$

for $\xi \in \mathrm{W} \Lambda$.

Let us show (i) by induction on $\operatorname{ht}(\Lambda-\mu)$. If $\mu=\Lambda$, it is trivial since $\chi_{i}\left(\mathrm{M}\left(s_{i} \Lambda\right)\right)=$ $\mathfrak{e}_{i}\left(\mathrm{M}\left(s_{i} \Lambda\right)\right)=a_{\Lambda, i}\left(t_{i}\right)$ by Proposition 3.6. Assume that $\mu \neq \Lambda$. 
If $\left\langle h_{i}, \mu\right\rangle<0$, then we have

$$
F_{i, \mu}\left(t_{i}\right)=F_{i, s_{i} \mu}\left(t_{i}\right)=\gamma \chi_{i}(\mathrm{M}(\mu)) \cdot \chi_{i}\left(\mathrm{M}\left(s_{i} \mu\right)\right) \quad \text { for some } \gamma \in \mathbf{k}^{\times},
$$

where the last equality follows from induction hypothesis applied to $s_{i} \mu$.

Hence we may assume that $\left\langle h_{i}, \mu\right\rangle \geq 0$. Set $\lambda=s_{i} \mu$. Take $j \in I$ such that $\left\langle h_{j}, \mu\right\rangle<0$, and set $\zeta=s_{j} \mu$. For such $\mu$ and $\lambda$, we shall show (i) and (ii). We set $l:=\left|\left\langle h_{i}, \zeta\right\rangle\right|$, $m:=\left\langle h_{i}, \mu\right\rangle$ and $n:=\left\langle h_{j}, \zeta\right\rangle$.

Note that

$$
\chi_{i, \zeta}\left(t_{i}\right)=\chi_{i, \mu}\left(t_{i}\right)
$$

By the definitions, $\mathfrak{e}_{i, \lambda}\left(t_{i}\right)$ and $\mathbf{q}_{i, j, \mu}\left(t_{i}\right)$ are quasi-monic, and

$$
\operatorname{deg} \mathfrak{e}_{i, \lambda}\left(t_{i}\right)=2\left(\alpha_{i}, \mu\right), \quad \operatorname{deg} \mathbf{q}_{i, j, \mu}\left(t_{i}\right)=2\left(\alpha_{i}, \mu-\zeta\right)
$$

(Case 1): Assume that $l=\left\langle h_{i}, \zeta\right\rangle \geq 0$. Note that $\operatorname{deg}\left(\mathfrak{e}_{i, \lambda}\left(t_{i}\right)\right)=\operatorname{deg}\left(\mathfrak{q}_{i, j, \mu}\left(t_{i}\right) \mathfrak{e}_{i, s_{i} \zeta}\left(t_{i}\right)\right)$ by (3.7). Thus, by Lemma 3.5, in order to see (ii), it suffices to show that

$$
\mathbf{q}_{i, j, \mu}\left(x_{m}\right) \mathfrak{e}_{i, s_{i} \zeta}\left(x_{m}\right) u=0 \quad \text { for any } u \in e\left(i^{m}, *\right) \mathrm{M}(\lambda)
$$

Let us first show

$$
\mathfrak{e}_{i, s_{i} \zeta}\left(x_{1}\right) e(i, *) R_{A}^{a_{\Lambda}}\left(\zeta-\alpha_{i}\right) e(i, *)=0 .
$$

If $l=\left\langle h_{i}, \zeta\right\rangle=0$, then $R_{A}^{a_{\Lambda}}\left(\zeta-\alpha_{i}\right) \simeq 0$ and (3.8) holds. Assume $\left\langle h_{i}, \zeta\right\rangle>0$. Lemma 3.5 implies that $\mathfrak{e}_{i, s_{i} \zeta}\left(x_{l}\right) e\left(i^{l}, *\right) \mathrm{M}\left(s_{i} \zeta\right)=0$. Since $\mathrm{M}\left(s_{i} \zeta\right)$ is a unique indecomposable projective $R_{A}^{a_{\Lambda}}\left(s_{i} \zeta\right)$-module,

$$
\mathfrak{e}_{i, s_{i} \zeta}\left(x_{l}\right) e\left(i^{l}, *\right) R_{A}^{a_{\Lambda}}\left(s_{i} \zeta\right)=0 .
$$

On the other hand, we have by [22, Lemma 4.14]

$$
E_{i}^{a_{\Lambda}(l-1)} F_{i}^{a_{\Lambda}(l)} R_{A}^{a_{\Lambda}}(\zeta) \simeq F_{i}^{a_{\Lambda}} R_{A}^{a_{\Lambda}}(\zeta) \simeq R_{A}^{a_{\Lambda}}\left(\zeta-\alpha_{i}\right) e(i, *)
$$

up to grading shifts. Since $e(i, *) R_{A}^{a_{\Lambda}}\left(\zeta-\alpha_{i}\right)$ is a direct summand of $E_{i}^{a_{\Lambda} l} F_{i}^{a_{\Lambda} l} R_{A}^{a_{\Lambda}}(\zeta) \simeq$ $e\left(i^{l}, *\right) R_{A}^{a_{\Lambda}}\left(s_{i} \zeta\right) e\left(i^{l}, *\right)$, we obtain $(3.8)$.

Then, we have

$$
\mathfrak{e}_{i, s_{i} \zeta}\left(x_{m+n}\right) e\left(i^{m-1}, j^{n}, i, *\right) \mathrm{M}(\lambda)=0,
$$


because $e\left(i^{m-1}, j^{n}, i, *\right) \mathrm{M}(\lambda)$ is an $e\left(i^{m-1}, j^{n}\right) \otimes e(i, *) R_{A}^{a_{\Lambda}}\left(\zeta-\alpha_{i}\right) e(i, *)$-module. Hence, for any $u \in e\left(i^{m}, j^{n}, *\right) \mathrm{M}(\lambda)$, we have

$$
\begin{aligned}
0 & =\left(\tau_{m} \tau_{m+1} \cdots \tau_{m+n-1}\right) \mathfrak{e}_{i, s_{i} \zeta}\left(x_{m+n}\right)\left(\tau_{m+n-1} \cdots \tau_{m+1} \tau_{m}\right) u \\
& =\mathfrak{e}_{i, s_{i} \zeta}\left(x_{m}\right)\left(\prod_{k=1}^{n} \mathcal{Q}_{i, j}\left(x_{m}, x_{m+k}\right)\right) u \\
& =\mathfrak{e}_{i, s_{i} \zeta}\left(x_{m}\right) \mathbf{q}_{i, j, \mu}\left(x_{m}\right) u
\end{aligned}
$$

Thus, we have proved (ii).

Let us show (i). By the induction hypothesis, we have $F_{i, \zeta}\left(t_{i}\right)=\gamma \chi_{i, \zeta}\left(t_{i}\right) \chi_{i, s_{i} \zeta}\left(t_{i}\right)$ for some $\gamma \in \mathbf{k}^{\times}$. Since $F_{i, \mu}\left(t_{i}\right)=\mathrm{q}_{i, j, \mu}\left(t_{i}\right) F_{i, \zeta}\left(t_{i}\right)$, we have, up to a constant multiple,

$$
\begin{aligned}
F_{i, \mu}\left(t_{i}\right) & =\mathrm{q}_{i, j, \mu}\left(t_{i}\right) F_{i, \zeta}\left(t_{i}\right)=\mathrm{q}_{i, j, \mu}\left(t_{i}\right) \chi_{i, \zeta}\left(t_{i}\right) \chi_{i, s_{i} \zeta}\left(t_{i}\right)=\mathrm{q}_{i, j, \mu}\left(t_{i}\right) \chi_{i, \zeta}\left(t_{i}\right) \mathfrak{e}_{i, s_{i} \zeta}\left(t_{i}\right) \chi_{i, \zeta}\left(t_{i}\right) \\
& =\mathfrak{e}_{i, \lambda}\left(t_{i}\right) \chi_{i, \mu}\left(t_{i}\right) \chi_{i, \mu}\left(t_{i}\right)=\chi_{i, s_{i} \mu}\left(t_{i}\right) \chi_{i, \mu}\left(t_{i}\right) .
\end{aligned}
$$

Here the second equality follows from the induction hypothesis applied to $\zeta$, and the fourth equality follows from (ii) and (3.6).

(Case 2): We assume that $\left\langle h_{i}, \zeta\right\rangle<0$. Since $e(i, *) \mathrm{M}(\mu)=0$, we have

$$
0=\left(\tau_{n} \cdots \tau_{2} \tau_{1}\right)\left(\tau_{1} \tau_{2} \cdots \tau_{n}\right) v=\left(\prod_{k=1}^{n} \mathcal{Q}_{i, j}\left(x_{n+1}, x_{k}\right)\right) v=\mathrm{q}_{i, j, \mu}\left(x_{n+1}\right) v
$$

for any $v \in e\left(j^{n}, i, *\right) \mathrm{M}(\mu)$. Hence we have $\mathrm{q}_{i, j, \mu}\left(x_{1}\right) e(i, *) \mathrm{M}(\zeta)=0$.

Thus, Lemma 3.5 implies that

$$
\mathfrak{e}_{i, \zeta}\left(t_{i}\right) \text { divides } \mathbf{q}_{i, j, \mu}\left(t_{i}\right) .
$$

We shall set

$$
S_{i, \mu}\left(t_{i}\right):=\left(\chi_{i, \mu}\left(t_{i}\right)\right)^{2}
$$

Then (i) is equivalent to

$$
F_{i, \mu}\left(t_{i}\right)=\mathfrak{e}_{i, \lambda}\left(t_{i}\right) S_{i, \mu}\left(t_{i}\right) .
$$

Now we have, by (3.6) and the induction hypothesis,

$$
\begin{aligned}
F_{i, \mu}\left(t_{i}\right) & =\mathrm{q}_{i, j, \mu}\left(t_{i}\right) F_{i, \zeta}\left(t_{i}\right)=\mathrm{q}_{i, j, \mu}\left(t_{i}\right) \chi_{i, \zeta}\left(t_{i}\right) \chi_{i, s_{i} \zeta}\left(t_{i}\right) \\
& =\mathrm{q}_{i, j, \mu}\left(t_{i}\right) \chi_{i, \zeta}\left(t_{i}\right)^{2} \mathfrak{e}_{i, \zeta}\left(t_{i}\right)^{-1}=\mathrm{q}_{i, j, \mu}\left(t_{i}\right) \mathfrak{e}_{i, \zeta}\left(t_{i}\right)^{-1} S_{i, \mu}\left(t_{i}\right) .
\end{aligned}
$$

Note that $\mathrm{q}_{i, j, \mu}\left(t_{i}\right) \mathfrak{e}_{i, \zeta}\left(t_{i}\right)^{-1}$ belongs to $A\left[t_{i}\right]$ by (3.10).

We now shall use results of [11] with $\beta=\Lambda-\mu{ }^{1}$

\footnotetext{
${ }^{1}$ The convention of $F_{i}, E_{i}$ in [11] is different from ours. The notation $E_{i} M$ is $e(*, i) M$ in [11] and $e(i, *) M$ in our paper, etc.
} 
In the diagram $[11,(5.6)], K_{1}^{\prime}, K_{0}^{\prime}$ and $F_{i}^{\Lambda} E_{i}^{\Lambda} R^{\Lambda}(\beta)$ in the first row vanish. Hence the diagram reduces (under our notations) to

$$
0 \longrightarrow q^{2\left(\alpha_{i}, \mu\right)} A\left[t_{i}\right] \otimes_{A} R_{A}^{a_{\Lambda}}(\mu) \stackrel{\mathscr{A}}{\longrightarrow} A\left[t_{i}\right] \otimes_{A} R_{A}^{a_{\Lambda}}(\mu) \stackrel{p}{\longrightarrow} e(i, *) R_{A}^{a_{\Lambda}}\left(\mu-\alpha_{i}\right) e(i, *) \longrightarrow 0 .
$$

Here $\mathscr{A}$ is an $R_{A}^{a_{\Lambda}}(\mu)$-bilinear map ${ }^{2}$ and $p$ is given by $p\left(f\left(t_{i}\right) \otimes a\right)=f\left(x_{1}\right) e(i) \otimes a$.

Under the notation in $[11,(5.10)], \varphi_{0}\left(t_{i}\right):=\mathscr{A}(1)$ commutes with $R_{A}^{a_{\Lambda}}(\mu)$. Hence $\varphi_{0}\left(t_{i}\right) \in A\left[t_{i}\right]$. Since $\varphi_{0}\left(t_{i}\right)$ is the image of $\mathscr{A}$, we have $\varphi_{0}\left(x_{1}\right) e(i, *) R_{A}^{a_{\Lambda}}\left(\mu-\alpha_{i}\right) e(i, *)=$ 0 . Setting $m=\left\langle h_{i}, \mu\right\rangle$, we can regard $e\left(i^{m}, *\right) \mathrm{M}\left(s_{i} \mu\right)$ as an $e(i, *) R_{A}^{a_{\Lambda}}\left(\mu-\alpha_{i}\right) e(i, *)$ module, and hence we have $\varphi_{0}\left(x_{m}\right) e\left(i^{m}, *\right) \mathrm{M}\left(s_{i} \mu\right)=0$. Then Lemma 3.5 implies that $\mathfrak{e}_{i, s_{i} \mu}\left(t_{i}\right)$ divides $\varphi_{0}\left(t_{i}\right)$. Since $\varphi_{0}\left(t_{i}\right)$ is a quasi-monic polynomial in $t_{i}$ of degree $\left\langle h_{i}, \mu\right\rangle$ by [11, Proposition 5.3], $\varphi_{0}\left(t_{i}\right)$ coincides with $\mathfrak{e}_{i, s_{i} \mu}\left(t_{i}\right)$ up to constant multiples.

By [11, Lemma 5.5], we have

$$
F_{i, \mu}\left(t_{i}\right)=\gamma \varphi_{0}\left(t_{i}\right) S_{i, \mu}\left(t_{i}\right)+\left(\text { a polynomial in } t_{i} \text { of degree }<\operatorname{deg}_{t_{i}} S_{i, \mu}\left(t_{i}\right)\right),
$$

for some $\gamma \in \mathbf{k}^{\times}$. Hence (3.12) implies that

$$
\mathfrak{e}_{i, s_{i} \mu}\left(t_{i}\right)=\varphi_{0}\left(t_{i}\right)=\mathrm{q}_{i, j, \mu}\left(t_{i}\right) \mathfrak{e}_{i, \zeta}\left(t_{i}\right)^{-1} \quad \text { up to a constant multiple, }
$$

and $F_{i, \mu}\left(t_{i}\right)=\mathfrak{e}_{i, s_{i} \mu}\left(t_{i}\right) S_{i, \mu}\left(t_{i}\right)$ up to a constant multiple.

Let $z$ be an indeterminate of degree $d \in 2 \mathbb{Z}_{>0}$ such that $2\left(\alpha_{i}, \Lambda\right) \in d \mathbb{Z}$, and set

$$
A=\mathbf{k}[z] \text {. }
$$

We take a family $a_{\Lambda}=\left\{a_{\Lambda, i}\left(t_{i}\right)\right\}$ of parameters as in Section 1.3 such that $a_{\Lambda, i}\left(t_{i}\right) \in A\left[t_{i}\right]$ is a monic polynomial and

$$
a_{\Lambda, i}(0) \in \mathbf{k}^{\times} z^{2\left(\alpha_{i}, \Lambda\right) / d} .
$$

Then one can consider the cyclotomic quiver Hecke algebra and the modules $\mathrm{M}\left(\lambda, \mu ; a_{\Lambda}\right)$ for $\lambda, \mu \in \mathrm{W} \Lambda$.

Theorem 3.26. For $\lambda, \mu \in \mathrm{W} \Lambda$ with $\lambda \preceq \mu, \mathrm{M}\left(\lambda, \mu ; a_{\Lambda}\right)$ is an affinization of $\mathrm{M}(\lambda, \mu)$.

Proof. We shall first treat the case $\mu=\Lambda$. We know already that $\mathrm{M}\left(\lambda, \Lambda ; a_{\Lambda}\right)$ is a finitely generated projective $A$-module, and $\mathbf{k} \otimes_{A} \mathrm{M}\left(\lambda, \Lambda ; a_{\Lambda}\right) \simeq \mathrm{M}(\lambda, \Lambda)$. Hence we have an exact sequence

$$
0 \rightarrow q^{d} \mathrm{M}\left(\lambda, \Lambda ; a_{\Lambda}\right) \stackrel{z}{\rightarrow} \mathrm{M}\left(\lambda, \Lambda ; a_{\Lambda}\right) \rightarrow \mathrm{M}(\lambda, \Lambda) \rightarrow 0 .
$$

The only remaining condition which we have to check is that $\left.\mathfrak{p}_{i}\right|_{\mathrm{M}\left(\lambda, \Lambda ; a_{\Lambda}\right)} \neq 0$.

We shall use induction on $\mathrm{ht}(\Lambda-\lambda)$. Since the case $\lambda=\Lambda$ is trivial, we may assume that $\lambda \neq \Lambda$. If there exists $j \in I$ such that $j \neq i$ and $\left\langle h_{j}, \lambda\right\rangle<0$, then we have

\footnotetext{
${ }^{2}$ The homomorphism $\mathscr{A}$ is denoted by $A$ in [11].
} 
$\left.\mathfrak{p}_{i}\right|_{\mathrm{M}\left(\lambda, \Lambda ; a_{\Lambda}\right)}=\left.\mathfrak{p}_{i}\right|_{\mathrm{M}\left(s_{j} \lambda, \Lambda ; a_{\Lambda}\right)}$ does not vanish by the induction hypothesis applied to $s_{j} \lambda$. Hence, we may assume that $\left\langle h_{i}, \lambda\right\rangle<0$. We have

$$
\left.\mathfrak{p}_{i}\right|_{\mathrm{M}\left(\lambda, \Lambda ; a_{\Lambda}\right)}= \pm \chi_{i}\left(\mathrm{M}\left(\lambda, \Lambda ; a_{\Lambda}\right)\right)(0) .
$$

By Proposition $3.25(\mathrm{i}), \chi_{i}\left(\mathrm{M}\left(\lambda, \Lambda ; a_{\Lambda}\right)\right)\left(t_{i}\right)$ divides $F_{i, \lambda}\left(t_{i}\right)$. Hence we have

$$
F_{i, \lambda}(0) \in \mathbf{k} \chi_{i}\left(\mathrm{M}\left(\lambda, \Lambda ; a_{\Lambda}\right)\right)(0) .
$$

Hence it is enough to show that $F_{i, \lambda}(0)$ does not vanish. We have $F_{i, \lambda}\left(t_{i}\right)=F_{i, s_{i} \lambda}\left(t_{i}\right)$ and $F_{i, s_{i} \lambda}(0)$ is a product of $a_{i, \Lambda}(0)$, a power of $\chi_{j}\left(\mathrm{M}\left(s_{i} \lambda, \Lambda ; a_{\Lambda}\right)\right)(0)(j \in I)$ and an element of $\mathbf{k}^{\times}$. The induction hypothesis applied to $s_{i} \lambda$ shows that they does not vanish. Hence $F_{i, \lambda}(0)$ does not vanish.

The case $\mu \prec \Lambda$ follows from Lemma 3.3 applied to $\mathrm{M}\left(\lambda, \Lambda ; a_{\Lambda}\right)$.

Proposition 3.27 (cf. [14, Proposition 10.2.3]). Let $\lambda, \mu \in \mathrm{P}_{+}$, and $s, s^{\prime}, t, t^{\prime} \in \mathrm{W}$ such that $\ell\left(s^{\prime} s\right)=\ell\left(s^{\prime}\right)+\ell(s), \ell\left(t^{\prime} t\right)=\ell\left(t^{\prime}\right)+\ell(t), s^{\prime} s \lambda \preceq t^{\prime} \lambda$, and $s^{\prime} \mu \preceq t^{\prime} t \mu$. Then we obtain

(i) $\mathrm{M}\left(s^{\prime} s \lambda, t^{\prime} \lambda\right)$ and $\mathrm{M}\left(s^{\prime} \mu, t^{\prime} t \mu\right)$ strongly commute,

(ii) $\Lambda\left(\mathrm{M}\left(s^{\prime} s \lambda, t^{\prime} \lambda\right), \mathrm{M}\left(s^{\prime} \mu, t^{\prime} t \mu\right)\right)=\left(s^{\prime} s \lambda+t^{\prime} \lambda, t^{\prime} t \mu-s^{\prime} \mu\right)$,

(iii) $\tilde{\Lambda}\left(\mathrm{M}\left(s^{\prime} s \lambda, t^{\prime} \lambda\right), \mathrm{M}\left(s^{\prime} \mu, t^{\prime} t \mu\right)\right)=\left(t^{\prime} \lambda, t^{\prime} t \mu-s^{\prime} \mu\right)$,

(iv) $\widetilde{\Lambda}\left(\mathrm{M}\left(s^{\prime} \mu, t^{\prime} t \mu\right), \mathrm{M}\left(s^{\prime} s \lambda, t^{\prime} \lambda\right)\right)=\left(s^{\prime} \mu-t^{\prime} t \mu, s^{\prime} s \lambda\right)$.

Proof. By [14, Proposition 9.1.6] (cf. [5, (10.2)]), we have

$$
\mathrm{D}\left(s^{\prime} s \lambda, t^{\prime} \lambda\right) \mathrm{D}\left(s^{\prime} \mu, t^{\prime} t \mu\right)=q^{\left(s^{\prime} s \lambda+t^{\prime} \lambda, s^{\prime} \mu-t^{\prime} t \mu\right)} \mathrm{D}\left(s^{\prime} \mu, t^{\prime} t \mu\right) \mathrm{D}\left(s^{\prime} s \lambda, t^{\prime} \lambda\right) .
$$

Thus, the assertions follow from $[\mathrm{M}(\lambda, \mu)]=\mathrm{D}(\lambda, \mu)([15$, Proposition 4.1]) and Lemma 3.20 .

\section{BRAiders in $R$-gmod}

4.1. Non-degenerate braiders. Let $R$ be a quiver Hecke algebra of arbitrary type. Then any module has a structure of braiders in $R$-gmod. We set $\Lambda=Q_{+}$.

Proposition 4.1. Let $\beta \in \mathrm{Q}_{+}$, and let $M$ be a simple $R(\beta)$-module. We assume that, for every $i \in I$, an $\left(R\left(\beta+\alpha_{i}\right), R\left(\alpha_{i}\right)\right)$-bilinear homomorphism $R_{i}: M \circ R\left(\alpha_{i}\right) \rightarrow$ $q^{\phi_{i}} R\left(\alpha_{i}\right) \circ M$ is given. Then, there exists a graded braider $\left(M, R_{M}, \phi\right)$ in $R$-gmod such that the morphism $R_{M}\left(R\left(\alpha_{i}\right)\right): M \circ R\left(\alpha_{i}\right) \rightarrow q^{\phi\left(\alpha_{i}\right)} R\left(\alpha_{i}\right) \circ M$ coincides with $R_{i}$ for every $i \in I$. Moreover, such a graded braider is unique up to an isomorphism. 
Proof. For $\alpha \in \mathrm{Q}_{+}$with $n=\mathrm{ht}(\alpha)$, we set

$$
\widetilde{R}(\alpha):=\mathbf{k}\left[x_{1}^{ \pm 1}, \ldots, x_{n}^{ \pm 1}\right] \otimes_{\mathbf{k}\left[x_{1}, \ldots, x_{n}\right]} R(\alpha) .
$$

The algebra structure of $\widetilde{R}(\alpha)$ is given similarly to $R(\alpha)$ with the use of (1.1).

For $i \in I$, we set $\widetilde{R}_{i}:=R_{i} \otimes_{R\left(\alpha_{i}\right)} \widetilde{R}\left(\alpha_{i}\right): M \circ \widetilde{R}\left(\alpha_{i}\right) \rightarrow \widetilde{R}\left(\alpha_{i}\right) \circ M$. Then one has $\widetilde{R}_{i}=c_{i} z_{i}^{m_{i}} \mathrm{R}_{M, \widetilde{R}\left(\alpha_{i}\right)}$ for some $c_{i} \in \mathbf{k}$ and $m_{i} \in \mathbb{Z}$ by Proposition 3.13 (iii). Here, $z_{i} \in \operatorname{End}_{\widetilde{R}\left(\alpha_{i}\right)}\left(\widetilde{R}\left(\alpha_{i}\right)\right)$ is the right multiplication by $x_{1}$. For any $\gamma \in \mathrm{Q}_{+} \operatorname{with} \operatorname{ht}(\gamma)=m$, we define $R_{M}(\widetilde{R}(\gamma))$ by $a_{\gamma} \mathrm{R}_{M, \widetilde{R}(\gamma)}$, where

$$
a_{\gamma}=\sum_{\nu \in I^{\gamma}}\left(\prod_{k=1}^{n} c_{\nu_{k}} x_{k}^{m_{\nu_{k}}} e(\nu)\right) \in \widetilde{R}(\gamma) .
$$

Since $a_{\gamma}$ belongs to the center of $\widetilde{R}(\gamma), R_{M}(\widetilde{R}(\gamma))$ is an $(R(\beta+\gamma), R(\gamma))$-bilinear homomorphism. Since it sends $M \circ R(\gamma)$ to $R(\gamma) \circ M$, we obtain an $(R(\beta+\gamma), R(\gamma))$ bilinear homomorphism $R_{M}(R(\gamma)): M \circ R(\gamma) \rightarrow R(\gamma) \circ M$. Thus, it is enough to define $R_{M}(X)=R_{M}(R(\gamma)) \otimes_{R(\gamma)} X: M \circ X \rightarrow X \circ M$.

The uniqueness is obvious by the construction.

Definition 4.2. Let $M$ be a simple $R$-module. A graded braider $\left(M, R_{M}, \phi\right)$ in $R$-gmod is non-degenerate if $R_{M}(L(i)): M \circ L(i) \rightarrow q^{\phi\left(\alpha_{i}\right)} L(i) \circ M$ is a non-zero homomorphism.

By Proposition 4.1 and the construction of a graded braider there, we have the following uniqueness statement.

Lemma 4.3. Let $M$ be a simple $R$-module. Then we have

(i) there exists a non-degenerate graded braider structure $(M, R, \phi)$,

(ii) if $\left(M, R^{\prime}, \phi^{\prime}\right)$ is another non-degenerate graded braider structure, then $\phi=\phi^{\prime}$ and there exists a group homomorphism $c: \mathbf{Q} \rightarrow \mathbf{k}^{\times}$such that $R^{\prime}(X)=c(\beta) R(X)$ for any $X \in R(\beta)$-gmod.

For a non-degenerate graded braider $(M, R, \phi)$, one has

$$
R_{M}\left(R\left(\alpha_{i}\right)\right)=\mathrm{R}_{M, R\left(\alpha_{i}\right)}^{\text {norm }} \text { up to a constant multiple. }
$$

Note that we have

$$
\phi\left(\alpha_{i}\right)=-\Lambda(M, L(i)) \text { for a non-degenerate graded braider }(M, R, \phi) .
$$

Proposition 4.4. Let $M$ be a simple module which is real and admits an affinization. Let $\left(M, R_{M}, \phi\right)$ be a non-degenerate graded braider in $R$-gmod. Let $\beta \in Q_{+}$and let $N$ be a simple $R(\beta)$-module. Then we have 
(i) $\Lambda(M, N) \leq-\phi(\beta)$.

(ii) The morphism $R_{M}(N): M \circ N \rightarrow q^{\phi(\beta)} N \circ M$ vanishes if $\Lambda(M, N)<-\phi(\beta)$, and does not vanish if $\Lambda(M, N)=-\phi(\beta)$.

Proof. We argue by induction on ht $(\beta)$. If $\beta=0$, then the assertion is trivial. If $\beta \neq 0$ take $i \in I$ such that $\varepsilon_{i}(N) \neq 0$. Then set $N_{0}=\widetilde{E}_{i}(N)$. Then we have $N \simeq L(i) \nabla N_{0}$. By Lemma 3.16, we have

$$
\Lambda(M, N) \leq \Lambda(M, L(i))+\Lambda\left(M, N_{0}\right) \leq-\phi\left(\alpha_{i}\right)-\phi\left(\beta-\alpha_{i}\right)=-\phi(\beta) .
$$

Hence we obtain (i).

Let us show (ii). If $\Lambda(M, N)<-\phi(\beta)$, then $R_{M}(N)=0$ since $\operatorname{Hom}(M \circ N, N \circ M)=$ $\mathbf{k r}_{M, N}$ is concentrated in degree $\Lambda(M, N)$.

Assume that $\Lambda(M, N)=-\phi(\beta)$. Then $\Lambda\left(M, N_{0}\right)=-\phi\left(\beta-\alpha_{i}\right)$ and $\Lambda(M, N)=$ $\Lambda(M, L(i))+\Lambda\left(M, N_{0}\right)$ by (4.3). Hence $R_{M}\left(N_{0}\right)$ is equal to $\mathbf{r}_{M, N_{0}}$ up to a constant multiple, and $R_{M}(N)=R_{M}\left(N_{0}\right) \circ R_{M}(L(i))$ is equal to $\mathbf{r}_{M, N}$ up to a constant multiple by Lemma 3.16 .

Corollary 4.5. Let $M$ be a simple module which is real and admits an affinization, and let $\left(M, R_{M}, \phi\right)$ be a non-degenerate graded braider in $R$-gmod. Let $N, N^{\prime}$ and $L$ be simple modules, and assume that there is an epimorphism $N \circ N^{\prime} \rightarrow L$. If $R_{M}(L)$ is non-zero, then so are $R_{M}(N)$ and $R_{M}\left(N^{\prime}\right)$. Equivalently, if we have $\Lambda(M, L)=$ $\phi(\operatorname{wt}(L))$, then $\phi(\operatorname{wt}(N))=\Lambda(M, N)$ and $\phi\left(\operatorname{wt}\left(N^{\prime}\right)\right)=\Lambda\left(M, N^{\prime}\right)$.

Note that $L \simeq N \nabla N^{\prime}$ when either $N$ or $N^{\prime}$ is real and admits an affinization.

Proof. By Lemma 3.16, we have

$$
\Lambda(M, N)+\Lambda\left(M, N^{\prime}\right) \geq \Lambda(M, L)=\phi(\operatorname{wt}(N))+\phi\left(\operatorname{wt}\left(N^{\prime}\right)\right) \geq \Lambda(M, N)+\Lambda\left(M, N^{\prime}\right) .
$$

Hence we obtain $\phi(\operatorname{wt}(N))=\Lambda(M, N)$ and $\phi\left(\operatorname{wt}\left(N^{\prime}\right)\right)=\Lambda\left(M, N^{\prime}\right)$.

As seen below, the real commutativity of a family of non-degenerate graded braiders can be checked easily.

Lemma 4.6. Let $\left\{S_{a}\right\}_{a \in A}$ be a family of simple modules, and assume that every $S_{a}$ is real and admits an affinization. Let $\left(S_{a}, R_{S_{a}}, \phi_{a}\right)$ be the associated non-degenerate graded braiders. The family $\left\{\left(S_{a}, R_{S_{a}}, \phi_{a}\right)\right\}_{a \in A}$ is a real commuting family of graded braiders if and only if $\phi_{a}\left(\operatorname{wt}\left(S_{b}\right)\right)+\phi_{b}\left(\operatorname{wt}\left(S_{a}\right)\right)=0$ for any $a, b \in A$.

Proof. Assume that $\phi_{a}\left(\operatorname{wt}\left(S_{b}\right)\right)+\phi_{b}\left(\operatorname{wt}\left(S_{a}\right)\right)=0$ for any $a, b \in A$. Since $0 \leq$ $2 \mathrm{~d}\left(S_{a}, S_{b}\right)=\Lambda\left(S_{a}, S_{b}\right)+\Lambda\left(S_{b}, S_{a}\right) \leq \phi_{a}\left(\operatorname{wt}\left(S_{b}\right)\right)+\phi_{b}\left(\operatorname{wt}\left(S_{a}\right)\right)=0$, we have $\phi_{a}\left(\operatorname{wt}\left(S_{b}\right)\right)=$ 
$\Lambda\left(S_{a}, S_{b}\right)$ and $\mathfrak{D}\left(S_{a}, S_{b}\right)=0$. Hence $R_{S_{a}}\left(S_{b}\right)$ is an isomorphism for any $a, b$. Thus $\left\{\left(S_{a}, R_{S_{a}}, \phi_{a}\right)\right\}_{a \in A}$ is a real commuting family of graded braiders.

The converse can be proved similarly.

Example 4.7. We shall provide an example of real simple modules $C_{1}, C_{2}$ where the associated non-degenerate braiders form a real commuting family of braiders, but any associated non-degenerate braiders $\left(C_{k}, R_{C_{k}}, \phi_{k}\right)(k=1,2)$ cannot satisfy $R_{C_{k}}\left(C_{k}\right)=$ $\mathrm{id}_{C_{k} \circ C_{k}}(k=1,2)$ and $R_{C_{2}}\left(C_{1}\right) \circ R_{C_{1}}\left(C_{2}\right)=\mathrm{id}_{C_{1} \circ C_{2}}$ at once. This is the reason why we should employ (a), (b) in Definition 2.2 for the definition of real commuting family.

Take $\mathfrak{g}=A_{2}, I=\{1,2\}, \mathcal{Q}_{12}(u, v)=c_{1} u+c_{2} v$ for $c_{1}, c_{2} \in \mathbf{k}^{\times}$. Then $\overline{\mathcal{Q}}_{12}=c_{1}$ and $\overline{\mathcal{Q}}_{21}=c_{2}$. Let $C_{1}=\langle 12\rangle, C_{2}=\langle 21\rangle$. Here, the $R\left(\alpha_{1}+\alpha_{2}\right)$-module $C_{1}$ is the onedimensional vector space with a basis $\langle 12\rangle$ on which $R\left(\alpha_{1}+\alpha_{2}\right)$ acts by $e(1,2)\langle 12\rangle=$ $\langle 12\rangle, x_{k}\langle 12\rangle=0(k=1,2)$ and $\tau_{1}\langle 12\rangle=0$. The $R\left(\alpha_{1}+\alpha_{2}\right)$-module $C_{2}$ is similarly defined.

Then $R_{C_{1}}$ is defined as follows for some constants $a_{1}, a_{2} \in \mathbf{k}^{\times}$.

$$
\begin{aligned}
\left(R_{C_{1}}\left(L(1)_{z}\right)\right)\left(\langle 12\rangle \otimes 1_{z}\right) & =z^{-1} a_{1} \varphi_{2} \varphi_{1}\left(1_{z} \otimes\langle 12\rangle\right)=z^{-1} a_{1} \tau_{2}\left(\tau_{1} z+1\right)\left(1_{z} \otimes\langle 12\rangle\right) \\
& =a_{1} \tau_{2} \tau_{1}\left(1_{z} \otimes\langle 12\rangle\right), \\
\left(R_{C_{1}}\left(L(2)_{z}\right)\right)\left(\langle 12\rangle \otimes 2_{z}\right) & =a_{2} \varphi_{2} \varphi_{1}\left(2_{z} \otimes\langle 12\rangle\right)=a_{2}\left(\tau_{2}\left(x_{2}-x_{3}\right)+1\right) \tau_{1}\left(2_{z} \otimes\langle 12\rangle\right) \\
& =a_{2}\left(z \tau_{2}+1\right) \tau_{1}\left(2_{z} \otimes\langle 12\rangle\right) .
\end{aligned}
$$

Similarly $R_{C_{2}}$ is defined for some constants $b_{1}, b_{2} \in \mathbf{k}^{\times}$:

$$
\begin{aligned}
& \left(R_{C_{2}}\left(L(1)_{z}\right)\right)\left(\langle 21\rangle \otimes 1_{z}\right)=b_{1}\left(z \tau_{2}+1\right) \tau_{1}\left(1_{z} \otimes\langle 21\rangle\right), \\
& \left(R_{C_{2}}\left(L(2)_{z}\right)\right)\left(\langle 21\rangle \otimes 2_{z}\right)=b_{2} \tau_{2} \tau_{1}\left(2_{z} \otimes\langle 21\rangle\right) .
\end{aligned}
$$

By a calculation, we can see that

$$
\begin{aligned}
R_{C_{1}}\left(C_{1}\right) & =a_{1} a_{2} c_{1} \mathrm{id}_{C_{1} \circ C_{1}}, \\
R_{C_{2}}\left(C_{2}\right) & =b_{1} b_{2} c_{2} \mathrm{id}_{C_{2} \circ C_{2}}, \\
R_{C_{2}}\left(C_{1}\right) R_{C_{1}}\left(C_{2}\right) & =-a_{1} a_{2} b_{1} b_{2} c_{1} c_{2} \mathrm{id}_{C_{1} \circ C_{2}} .
\end{aligned}
$$

4.2. Localization. Let $\mathscr{C}$ be a full subcategory of $R$-gmod satisfying

$\mathscr{C}$ contains 1 and is stable by taking subquotients, convolutions, extensions and grading shifts.

Let $\left\{C_{a}\right\}_{a \in A}$ be a family of simple objects of $\mathscr{C}$. Let $\left(C_{a}, R_{C_{a}}, \phi_{a}\right)$ be non-degenerate graded braiders in $R$-gmod. Assume that every $C_{a}$ admits affinization.

Assume that $\left\{\left(C_{a}, R_{C_{a}}, \phi_{a}\right)\right\}_{a \in A}$ is a real commuting family of graded braiders. 
Set $\Gamma=\mathbb{Z}^{\oplus A}$ and $\Gamma_{\geq 0}=\mathbb{Z}_{\geq 0}^{\oplus A}$, and for $\alpha \in \Gamma_{\geq 0}$, let $C^{\alpha} \in \mathscr{C}$ be the object constructed in Subsection 2.3. Let $\widetilde{\mathscr{C}}:=\mathscr{C}\left[C_{a}^{\circ-1} \mid a \in A\right]$ be the localization of $\mathscr{C}$. We still denote by $\circ$ the tensor functor of $\tilde{\mathscr{C}}$. Let $\Phi: \mathscr{C} \rightarrow \widetilde{\mathscr{C}}$ be the canonical functor. They enjoy the following properties.

(a) $\widetilde{\mathscr{C}}$ is an abelian category and $\Phi$ is exact,

(b) $\widetilde{C}_{a}:=\Phi\left(C_{a}\right)$ is an invertible central graded braider in $\widetilde{\mathscr{C}}$.

We define $\widetilde{C}^{\alpha}$ in $\widetilde{\mathscr{C}}$ for $\alpha \in \Gamma$ such that $\widetilde{C}^{\alpha+\beta} \simeq \widetilde{C}^{\alpha} \circ \widetilde{C}^{\beta}$ (up to a grading shift) for $\alpha, \beta \in \Gamma$ and $\widetilde{C}^{\alpha}=\Phi\left(C^{\alpha}\right)$ for $\alpha \in \Gamma_{\geq 0}$. Then

(c) any object of $\tilde{\mathscr{C}}$ is isomorphic to $\widetilde{C}^{\alpha} \circ \Phi(X)$ for some $X \in \mathscr{C}$ and $\alpha \in \Gamma$.

Proposition 4.8. Let $\Phi: \mathscr{C} \rightarrow \widetilde{\mathscr{C}}$ be the canonical functor. Then we have the following properties.

(i) Let $S$ be a simple object of $\mathscr{C}$. The object $\Phi(S)$ is a simple object of $\tilde{\mathscr{C}}$ if $\phi_{a}(\operatorname{wt}(S))=\Lambda\left(C_{a}, S\right)$ for any $a \in A$. Otherwise, $\Phi(S)$ vanishes.

(ii) Any object of $\widetilde{\mathscr{C}}$ has finite length.

(iii) For simple modules $S, S^{\prime} \in \mathscr{C}$ such that $\Phi(S) \simeq \Phi\left(S^{\prime}\right) \not 40$, we have $S \simeq S^{\prime}$.

Proof. In the course of the proof, we ignore grading shifts.

(i) Let $S$ be a simple object of $\mathscr{C}$ such that $\phi_{a}(\operatorname{wt}(S)) \neq \Lambda\left(C_{a}, S\right)$ for some $a \in A$. Then $R_{C_{a}}(S): C_{a} \circ S \rightarrow S \circ C_{a}$ vanishes. Hence $\Phi\left(\operatorname{id}_{S}\right)=0$ and $\Phi(S) \simeq 0$.

Now assume that $\phi_{a}(\operatorname{wt}(S))=\Lambda\left(C_{a}, S\right)$ for any $a \in A$. Then $R_{C^{\alpha}}(S)$ does not vanish for any $\alpha \in \Gamma_{\geq 0}$ by Proposition 4.4. It means that $\Phi\left(\mathrm{id}_{S}\right)$ does not vanish. Hence $\Phi(S)$ does not vanish. Let us show that any subobject of $\Phi(S)$ is either zero or $\Phi(S)$. In order to see this, it is enough to show that for any $\alpha \in \Gamma_{\geq 0}$ and a non-zero morphism $f: X \rightarrow S \circ C^{\alpha}$ in $\mathscr{C}$, the morphism $\Phi(f): \Phi(X) \rightarrow \Phi\left(S \circ C^{\alpha}\right)$ is an epimorphism. The image $f(X)$ contains $S \Delta C^{\alpha}$. Hence $X^{\prime} \rightarrow S \Delta C^{\alpha}$ is an epimorphism where $X^{\prime}=f^{-1}\left(S \Delta C^{\alpha}\right) \subset X$. Hence $\Phi\left(X^{\prime}\right) \rightarrow \Phi\left(S \Delta C^{\alpha}\right)$ is an epimorphism. On the other hand, we have $\phi_{C_{\alpha}}(\operatorname{wt}(S))=\Lambda\left(C^{\alpha}, S\right)$, and hence $S \Delta C^{\alpha}$ coincides with the image of $R_{C_{\alpha}}(S)$. Since $\Phi\left(R_{C_{\alpha}}(S)\right)$ is an isomorphism, the morphism $\Phi\left(S \Delta C^{\alpha}\right) \rightarrow \Phi\left(S \circ C^{\alpha}\right)$ is an isomorphism. Hence $\Phi\left(X^{\prime}\right) \rightarrow \Phi\left(S \circ C^{\alpha}\right)$ is an epimorphism, which implies that $\Phi(X) \rightarrow \Phi\left(S \circ C^{\alpha}\right)$ is an epimorphism.

(ii) It is enough to show that $\Phi(X)$ has a finite length for any $X \in \mathscr{C}$. Let $0=X_{-1} \subset$ $X_{0} \subset \cdots \subset X_{n}=X$ be a composition series of $X$. Then (i) implies that $\Phi\left(X_{k} / X_{k-1}\right)$ is simple or vanishes. Hence $\Phi(X)$ has a finite length. 
(iii) By $\Phi\left(S^{\prime}\right) \not 40$, (i) implies that $\phi_{C^{\alpha}}\left(\operatorname{wt}\left(S^{\prime}\right)\right)=\Lambda\left(C_{\alpha}, S^{\prime}\right)$. If $\Phi(S) \simeq \Phi\left(S^{\prime}\right)$, then there exist $\alpha \in \Gamma_{\geq 0}$ and a non-zero morphism $C^{\alpha} \circ S \rightarrow q^{-\Lambda\left(C_{\alpha}, S^{\prime}\right)} S^{\prime} \circ C^{\alpha}$. Hence Proposition 3.22 implies that $S$ and $S^{\prime}$ are isomorphic.

Proposition 4.9. Assume that $A$ is a finite set. Let $\mathcal{Z}_{\mathscr{C}}\left(C_{a} \mid a \in A\right)$ be the full subcategory of $\mathscr{C}$ consisting of objects $X \in \mathscr{C}$ such that $R_{C_{a}}(X)$ is an isomorphism for any $a \in A$. Assume that $\alpha \in \Gamma$ satisfies $\alpha-e_{a} \in \Gamma_{\geq 0}$ for every $a \in A$, and set $C=C^{\alpha}$.

(i) $\mathcal{Z}_{R \text {-gmod }}\left(C_{a} \mid a \in A\right)$ contains 1 and is stable by taking subquotients, convolutions, extensions, grading shifts.

(ii) For $X \in \mathcal{C}, X \in \mathcal{Z}_{\mathscr{C}}\left(C_{a} \mid a \in A\right)$ if and only if $R_{C}(X)$ is an isomorphism.

(iii) Setting $\mathcal{Z}=\mathcal{Z}_{\mathscr{C}}\left(C_{a} \mid a \in A\right)$, the functor $\mathcal{Z}\left[C_{a}^{\circ-1} \mid a \in A\right] \longrightarrow \widetilde{\mathscr{C}}=\mathscr{C}\left[C_{a}^{\circ-1} \mid a \in\right.$ $A]$ is an equivalence of categories.

Proof. (i) Let us show that if $X \in \mathcal{Z}_{R \text {-gmod }}\left(C_{a} \mid a \in A\right)$, then any subobject $Y$ of $X$ belongs to $\mathcal{Z}_{R \text {-gmod }}\left(C_{a} \mid a \in A\right)$. For any $a \in A, R_{C_{a}}(X)$ is a monomorphism. Hence $R_{C_{a}}(Y): C_{a} \circ Y \rightarrow Y \circ C_{a}$ is a monomorphism. Since $C_{a} \circ Y$ and $Y \circ C_{a}$ have same dimension, $R_{C_{a}}(Y)$ is an isomorphism. The other properties of $\mathcal{Z}_{R \text {-gmod }}\left(C_{a} \mid a \in A\right)$ can be proved similarly.

(ii) It is obvious that $R_{C}(X)$ is an isomorphism for any $X \in \mathcal{Z}$. Conversely, assume that $R_{C}(X)$ is an isomorphism. Then $R_{C}(X)=\left(R_{C^{\alpha-e_{a}}}(X) \otimes C_{a}\right) \circ\left(C^{\alpha-e_{a}} \otimes R_{C_{a}}(X)\right)$ is injective, and hence $R_{C_{a}}(X)$ is injective. Then we conclude that it is an isomorphism.

(iii) We shall show that $\mathcal{Z}\left[C^{\otimes-1}\right] \rightarrow \mathscr{C}\left[C^{\otimes-1}\right]$ is essentially surjective (see Lemma 2.8). It is enough to show that for any $X \in \mathscr{C}, \Phi(X) \in \tilde{\mathscr{C}}$ is contained in the image of $\mathcal{Z}\left[C^{\otimes-1}\right]$. By Corollary 3.18, for $n \gg 0$, every simple subquotient of $C^{\circ} \circ X$ commutes with $C$. Hence, by replacing $X$ with $C^{\circ n} \circ X$, we may assume from the beginning that every simple subquotient of $X$ commutes with $C$. Then we have $\ell(X)=\ell\left(C^{\circ n} \circ X\right)=\ell\left(X \circ C^{\circ n}\right)$ for any $n \in \mathbb{Z}_{\geq 0}$. Here $\ell$ denote the length function. Since $\operatorname{Im}\left(R_{C^{\circ(m+n)}}(X)\right)=\operatorname{Im}\left(R_{C^{\circ m}}\left(\operatorname{Im} R_{C^{\circ n}}(X)\right)\right), \ell\left(\operatorname{Im} R_{C^{\circ n}}(X)\right)$ is a decreasing sequence in $n$. Hence, by replacing $X$ with $\operatorname{Im}\left(R_{C^{\circ} n}(X)\right)$ for $n \gg 0$, we may assume from the beginning that $\ell\left(\operatorname{Im} R_{C}(X)\right)=\ell(X)=\ell(C \circ X)$. Then we conclude that $R_{C}(X): C \circ X \rightarrow X \circ C$ is an isomorphism.

Example 4.10. Take $\mathfrak{g}=A_{2}, I=\{1,2\}$ and $C=L(1)$. Set $\widetilde{\mathscr{C}}=R$-gmod $\left[C^{\circ-1}\right]$ and $\Phi: R$-gmod $\longrightarrow \widetilde{\mathscr{C}}$ the canonical functor. Then $\Phi(L(2)) \simeq C^{\circ-1} \circ \Phi(L(1,2))$, $\Phi(L(2,1)) \simeq 0$ because $-1=\Lambda(C, L(2,1))<\phi_{C}(\operatorname{wt}(L(2,1))=\Lambda(C, L(2))+\Lambda(C, L(1))$ $=1$. Hence any simple object in $\widetilde{\mathscr{C}}$ is isomorphic to $L(1,2)^{\circ m} \circ C^{\circ n}$ for some $m \in \mathbb{Z}_{\geq 0}$ and $n \in \mathbb{Z}$. 
4.3. Left duals. In this subsection, we take $\beta \in \mathrm{Q}_{+}$, a simple $R(\beta)$-module $C$ and a real non-degenerate graded braider $(C, R, \phi)$. We assume that $C$ has an affinization. We shall consider the localization $(R-\operatorname{gmod})^{\sim}:=R$-gmod $\left[C^{\circ-1}\right]$.

Proposition 4.11. Let $X$ and $Y$ be simple $R$-modules. Let $\varepsilon: X \circ Y \rightarrow C$ be an epimorphism. Then we have

(i) $\phi(\operatorname{wt}(X))=\Lambda(C, X), \phi(\operatorname{wt}(Y))=\Lambda(C, Y)$, and $\Lambda(C, X)+\Lambda(X, C)=0$,

(ii) $R_{C}(X)$ is an isomorphism,

(iii) $\left(C^{\circ-1} \circ X, Y\right)$ is a dual pair in $(R \text {-gmod })^{\sim}$.

Proof. Since $\phi(\operatorname{wt}(C))=\Lambda(C, C)$, Corollary 4.5 implies that $\phi(\operatorname{wt}(X))=\Lambda(C, X)$ and $\phi(\operatorname{wt}(Y))=\Lambda(C, Y)$. Since $\phi(\operatorname{wt}(C))=0$, one has $\Lambda(C, X)+\Lambda(C, Y)=0$.

Taking the dual of $\varepsilon$ we have a monomorphism

$$
\eta: C \longmapsto q^{n} Y \circ X
$$

for some $n \in \mathbb{Z}$. Then by [14, Lemma 3.1.5 (i)], the compositions

$$
\begin{aligned}
& X \circ C \stackrel{\eta}{\longrightarrow} q^{n} X \circ Y \circ X \stackrel{\varepsilon}{\longrightarrow} q^{n} C \circ X, \\
& C \circ Y \stackrel{\eta}{\longrightarrow} q^{n} Y \circ X \circ Y \stackrel{\varepsilon}{\longrightarrow} q^{n} Y \circ C
\end{aligned}
$$

are non-zero. Hence, we have $\Lambda(X, C)=-n$ and $\Lambda(C, Y)=-n$. Hence $\Lambda(C, X)+$ $\Lambda(X, C)=0$. Thus we obtain (i). Note that we have

$$
n=\phi(\mathrm{wt}(X))=-\phi(\mathrm{wt}(Y)) \text {. }
$$

(ii) follows from (i).

(iii) Set $\widetilde{X}=C^{\circ-1} \circ X \in(R \text {-gmod })^{\sim}$. The isomorphism $R_{C}(X): C \circ X \rightarrow q^{-n} X \circ C$ in $(R \text {-gmod })^{\sim}$ induces $\tilde{X} \simeq q^{n} X \circ C^{\circ-1}$. Let

$$
\widetilde{\varepsilon}:=C^{\circ-1} \circ \varepsilon: \widetilde{X} \circ Y \rightarrow \mathbf{1} \text { and } \tilde{\eta}:=\eta \circ C^{\circ-1}: \mathbf{1} \rightarrow q^{n} Y \circ X \circ C^{-1} \simeq Y \circ \widetilde{X}
$$

be morphisms in $(R \text {-gmod })^{\sim}$.

Note that

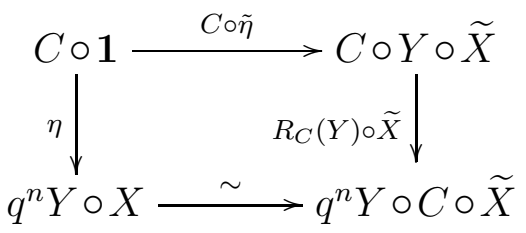

commutes up to a constant multiple. Indeed, after convoluting $C$ from the right, the diagram commutes up to a constant multiple, which follows from $R_{C}(C) \in \mathbf{k}^{\times} \mathrm{id}_{C \circ C}$ 
and the commutativity of the diagram below:

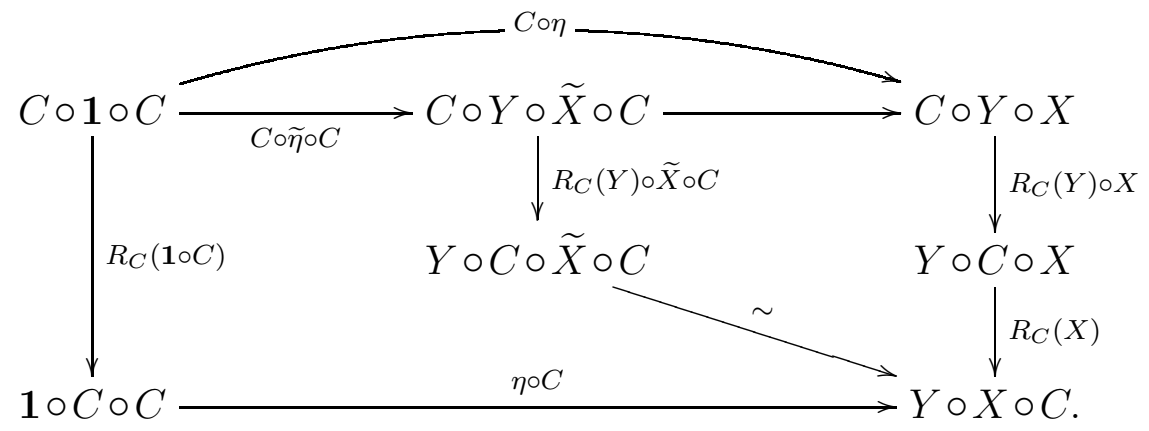

Let us show that $(\tilde{\varepsilon}, \tilde{\eta})$ is a quasi-adjunction.

The composition

$$
X \circ C \stackrel{X \circ \eta}{\longrightarrow} X \circ Y \circ X \stackrel{\varepsilon \circ X}{\longrightarrow} C \circ X
$$

is non-zero by [14, Lemma 3.1.5 (i)], and hence it is an isomorphism since $C \circ X$ and $X \circ C$ are simple by (ii). It implies that the composition

$$
\tilde{X} \circ 1 \stackrel{\widetilde{X} \circ \widetilde{\eta}}{\longrightarrow} \tilde{X} \circ Y \circ \widetilde{X} \stackrel{\widetilde{\varepsilon} \circ \widetilde{X}}{\longrightarrow} 1 \circ \widetilde{X}
$$

is an isomorphism.

Similarly, since $C \circ Y \stackrel{\eta \circ Y}{\longrightarrow} Y \circ X \circ Y \stackrel{Y \circ \varepsilon}{\longrightarrow} Y \circ C$ does not vanish, Proposition 3.13 (ii) implies the composition is equal to $R_{C}(Y)$ up to a constant multiple. Hence it is an isomorphism in $(R \text {-gmod })^{\sim}$. Hence, the composition

$$
\mathbf{1} \circ Y \stackrel{\widetilde{\eta} \circ Y}{\longrightarrow} Y \circ \widetilde{X} \circ Y \stackrel{Y \circ \widetilde{\varepsilon}}{\longrightarrow} Y \circ \mathbf{1}
$$

is also an isomorphism. Indeed, it is enough to show that it is an isomorphism after operating $C \circ *$, which follows from the following commutative diagram (up to constant multiples).

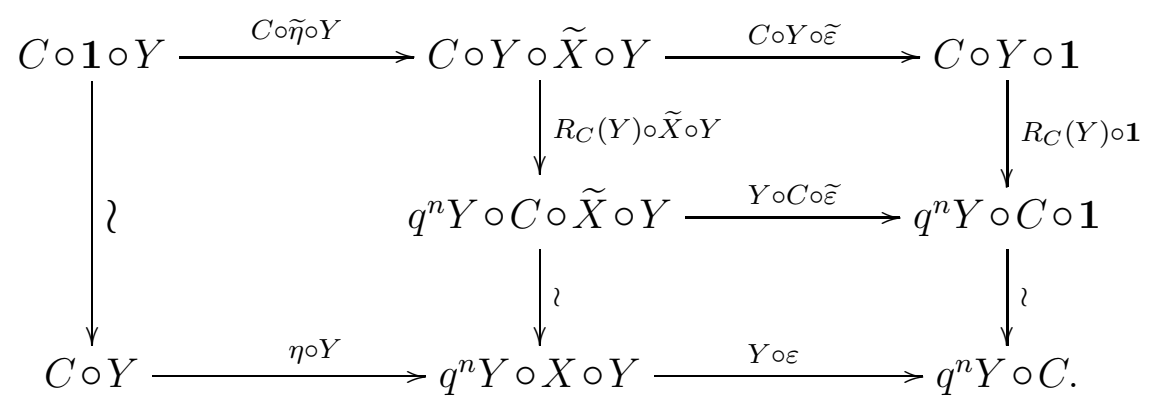


Let $i \in I$. Let $z$ be an indeterminate of degree $\left(\alpha_{i}, \alpha_{i}\right)$ and set $\widetilde{L}(i)=\mathbf{k}[z]\langle i\rangle_{z}$. Let $\widetilde{L}(i)$ endow with the $R\left(\alpha_{i}\right)$-module structure by $x_{1}\langle i\rangle_{z}=z\langle i\rangle_{z}$. Then $(\widetilde{L}(i), z)$ is an affinization of $L(i)$.

For $\ell \in \mathbb{Z}_{>0}$, we set $L_{\ell}(i)=\widetilde{L}(i) / z^{\ell} \widetilde{L}(i)$.

Theorem 4.12. Let $i \in I$ and assume that $\varepsilon_{i}^{*}(C)=1$. For $\ell \in \mathbb{Z}_{>0}$, we set $K_{\ell}=$ $E_{i}^{*}\left(C^{\circ \ell}\right)$. Then $C^{\circ-\ell} \circ K_{\ell}$ is a left dual of $L_{\ell}(i)$ in $(R \text {-gmod) })^{\sim}$.

The rest of this subsection is devoted to the proof of this theorem. Set $m=\operatorname{ht}(\beta)$.

For simplicity, we write

$$
L_{\ell}:=L_{\ell}(i) \text { and } C_{\ell}:=C^{\mathrm{o} \ell} \text {. }
$$

Note that $L_{1}(i)=L(i)$. We write $\langle i\rangle_{\ell} \in L_{\ell}$ for the image of $\langle i\rangle_{z} \in \widetilde{L}(i)$. Hence $L_{\ell}=R\left(\alpha_{i}\right)\langle i\rangle_{\ell}$.

We have an epimorphism $K_{1} \circ L_{1} \rightarrow C$. Hence, Proposition 4.11 implies that $C^{\circ-1} \circ$ $K_{1}$ is a left dual of $L_{1}$. The same proposition and Lemma 3.15 imply $\phi\left(\operatorname{wt}\left(L_{1}\right)\right)=$ $\Lambda\left(C, L_{1}\right)=-\left(\operatorname{wt}\left(K_{1}\right), \operatorname{wt}\left(L_{1}\right)\right)=-\left(\beta-\alpha_{i}, \alpha_{i}\right)$. Hence we obtain

$$
\phi\left(\alpha_{i}\right)=\left(\beta-\alpha_{i}, \alpha_{i}\right) .
$$

Lemma 4.13. There exist a surjective $R(\ell \beta)$-module homomorphism

$$
\varepsilon_{\ell}: K_{\ell} \circ L_{\ell} \rightarrow C_{\ell}
$$

and an injective $R(\ell \beta)$-module homomorphism

$$
\eta_{\ell}: C_{\ell} \longmapsto q^{\ell \phi\left(\alpha_{i}\right)} L_{\ell} \circ K_{\ell}
$$

Proof. Since $C_{\ell}$ is a simple module which satisfies $\varepsilon_{i}^{*}\left(C_{\ell}\right)=\ell$, we have

$$
\left(x_{\ell m}\right)^{\ell} e\left(\ell \beta-\alpha_{i}, i\right) C_{\ell}=0
$$

by Lemma 3.5 and $\mathfrak{e}_{i}^{*}\left(C_{\ell}\right)(t)=t^{\ell}$. Hence we obtain a non-zero morphism

$$
\varepsilon_{\ell}: K_{\ell} \circ L_{\ell} \rightarrow C_{\ell}
$$

by $u \otimes\left(x_{1}^{k}\langle i\rangle_{z}\right) \mapsto\left(x_{\ell m}\right)^{k} u$ for $u \in e(*, i) C_{\ell}$. It is an epimorphism.

Recall that $(M \circ N)^{\star} \simeq q^{(\operatorname{wt}(M), \operatorname{wt}(N))} N^{\star} \circ M^{\star}$ for $R$-modules $M$ and $N$. Taking the dual of $K_{\ell} \circ L_{\ell} \rightarrow C_{\ell}$, we have

$$
\left(C_{\ell}\right)^{\star} \longmapsto q^{\left(\alpha_{i}, \ell \beta-\alpha_{i}\right)} L_{\ell}^{\star} \circ\left(K_{\ell}\right)^{\star} \simeq q^{\left(\alpha_{i}, \ell \beta-\alpha_{i}\right)-(\ell-1)\left(\alpha_{i}, \alpha_{i}\right)} L_{\ell} \circ\left(K_{\ell}\right)^{\star} .
$$

Since $E_{i}^{*}$ commutes with the duality $\star$, setting $\left(C_{\ell}\right)^{\star} \simeq q^{a} C_{\ell}$, we obtain

$$
\left(K_{\ell}\right)^{\star} \simeq\left(E_{i}^{*} C_{\ell}\right)^{\star} \simeq E_{i}^{*}\left(\left(C_{\ell}\right)^{\star}\right) \simeq q^{a} E_{i}^{*}\left(C_{\ell}\right) \simeq q^{a} K_{\ell}
$$


Hence, we obtain

$$
C_{\ell} \longmapsto q^{\ell\left(\beta-\alpha_{i}, \alpha_{i}\right)} L_{\ell} \circ K_{\ell}=q^{\ell \phi\left(\alpha_{i}\right)} L_{\ell} \circ K_{\ell}
$$

Here the last equality follows from (4.7).

We now define

$$
\widetilde{K}_{\ell}:=\left(C_{\ell}\right)^{\circ(-1)} \circ K_{\ell} \in(R \text {-gmod })^{\sim} .
$$

Note that

$$
\widetilde{K}_{\ell} \simeq q^{-\ell \phi\left(\ell \beta-\alpha_{i}\right)} K_{\ell} \circ\left(C_{\ell}\right)^{\circ(-1)} \simeq q^{\ell \phi\left(\alpha_{i}\right)} K_{\ell} \circ\left(C_{\ell}\right)^{\circ(-1)} .
$$

Hence, Lemma 4.13 gives morphisms

$$
\widetilde{\varepsilon}_{\ell}: \widetilde{K}_{\ell} \circ L_{\ell} \rightarrow \mathbf{1} \text { and } \widetilde{\eta}_{\ell}: \mathbf{1} \longmapsto L_{\ell} \circ \widetilde{K}_{\ell} .
$$

We shall show that $\left(\widetilde{\varepsilon}_{\ell}, \widetilde{\eta}_{\ell}\right)$ is a quasi-adjunction by induction on $\ell$.

We know already that the assertion holds when $\ell=1$. Suppose that $\ell>1$ and $\left(\widetilde{\varepsilon}_{k}, \widetilde{\eta}_{k}\right)$ is a quasi-adjunction for $k<\ell$.

Let us take $a, b \in \mathbb{Z}_{>0}$ such that $\ell=a+b$. By the definition, we have the following short exact sequences:

$$
0 \longrightarrow q_{i}^{2 b} L_{a} \stackrel{z^{b}}{\longrightarrow} L_{\ell} \longrightarrow L_{b} \longrightarrow 0 \text {. }
$$

Here, $q_{i}=q^{\left(\alpha, \alpha_{i}\right) / 2}$, and $q_{i}^{2 b} L_{a} \stackrel{z^{b}}{\longrightarrow} L_{\ell}$ is given by $\langle i\rangle_{a} \mapsto z^{b}\langle i\rangle_{\ell}$.

For $M \in R(\beta)$-Mod and $N \in R(\gamma)$-Mod, there is a short exact sequence (see [18, Proposition 2.18])

$$
0 \longrightarrow M \circ\left(E_{i}^{*} N\right) \longrightarrow E_{i}^{*}(M \circ N) \longrightarrow q^{-\left(\alpha_{i}, \gamma\right)}\left(E_{i}^{*} M\right) \circ N \longrightarrow 0 .
$$

Applying this to $M=C_{a}$ and $N=C_{b}$, we obtain the exact sequence

$$
0 \longrightarrow C_{a} \circ K_{b} \stackrel{f}{\longrightarrow} K_{\ell} \stackrel{g}{\longrightarrow} q^{-b\left(\beta, \alpha_{i}\right)} K_{a} \circ C_{b} \longrightarrow 0 .
$$

By (4.7), we have

$$
K_{a} \circ C_{b} \simeq q^{-b \phi\left(a \beta-\alpha_{i}\right)} C_{b} \circ K_{a}=q^{b\left(\beta-\alpha_{i}, \alpha_{i}\right)} C_{b} \circ K_{a}
$$

in $(R \text {-gmod })^{\sim}$, which yields an exact sequence in $(R \text {-gmod })^{\sim}$ :

$$
0 \longrightarrow C_{a} \circ K_{b} \longrightarrow K_{\ell} \longrightarrow q^{-b\left(\alpha_{i} \alpha_{i}\right)} C_{b} \circ K_{a} \longrightarrow 0 .
$$

From $(4.11)$, we obtain an exact sequence in $(R \text {-gmod })^{\sim}$

$$
0 \longrightarrow \widetilde{K}_{b} \longrightarrow \widetilde{K}_{\ell} \longrightarrow q_{i}^{-2 b} \widetilde{K}_{a} \longrightarrow 0 .
$$


In order to see that $\left(\widetilde{\varepsilon}_{\ell}, \widetilde{\eta}_{\ell}\right)$ is a quasi-adjunction, we shall apply Lemma 1.15 to the short exact sequences (4.9) and (4.12). Hence it is enough to show that the following two diagrams in $(R$-gmod $) \sim$ commute up to constant multiples:

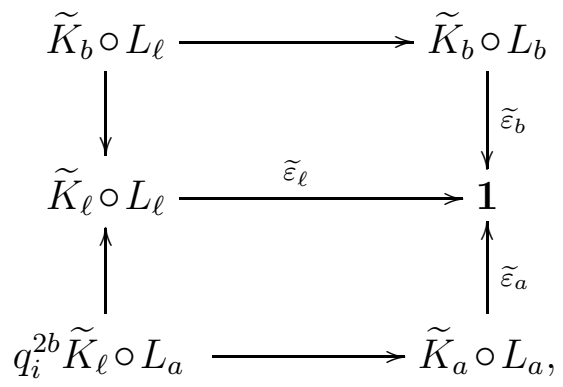

and

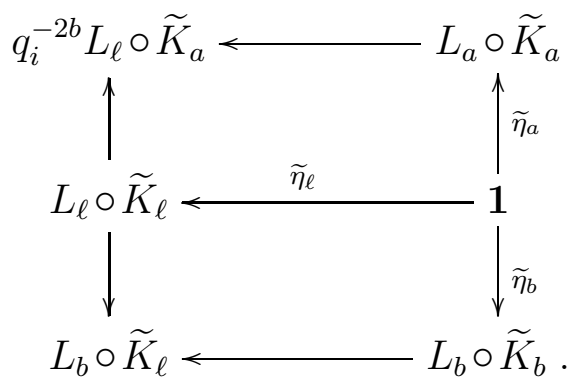

Since the diagram (4.14) is the dual of (4.13) after the exchange of $a$ and $b$, it is enough to show the commutativity (up to constant multiples) of (4.13). Then it is reduced to the commutativity (up to constant multiples) of the following two diagrams in $R$-gmod:

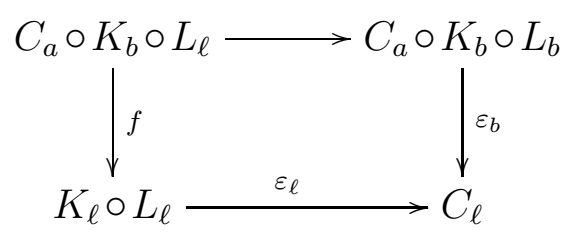

and

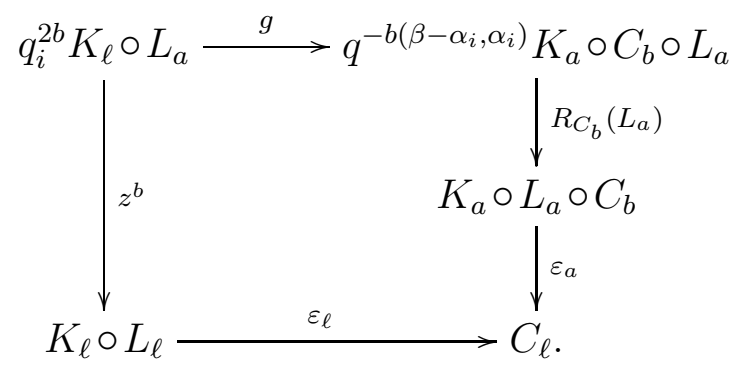

Let us first show the commutativity of (4.15). 
The module $C_{a} \circ K_{b} \circ L_{\ell}$ is generated by $s:=u \otimes v \otimes\langle i\rangle_{\ell}$ as an $R(\ell \beta)$-module. Here, $u \in C_{a}$ and $v \in e(*, i) C_{b}$. Then the element $s$ is sent by $C_{a} \circ K_{b} \circ L_{\ell} \rightarrow C_{a} \circ K_{b} \circ L_{b} \rightarrow$ $C_{\ell}$ as follows:

$$
u \otimes v \otimes\langle i\rangle_{\ell} \longmapsto u \otimes\left(v \otimes\langle i\rangle_{b}\right) \longmapsto u \otimes v .
$$

On the other hand, by $C_{a} \circ K_{b} \circ L_{\ell} \rightarrow K_{\ell} \circ L_{\ell} \rightarrow C_{\ell}$, the element $s$ is sent as follows:

$$
u \otimes v \otimes\langle i\rangle_{\ell} \longmapsto(u \otimes v) \otimes\langle i\rangle_{\ell} \longmapsto u \otimes v .
$$

Since these two images coincide, the diagram (4.15) commutes.

Now, let us show the commutativity (up to constant multiples) of (4.16).

To do it, we need an explicit construction of the morphism $g$. We regard $E_{i}^{*(b)} C_{b}$ as the subspace

$$
\left.T:=\left\{v \in e\left(b\left(\beta-\alpha_{i}\right), i^{b}\right) C_{b} \mid\left(e\left(b\left(\beta-\alpha_{i}\right)\right)\right) \otimes x_{k}\right) v=0 \text { for } 1 \leq k \leq b\right\} \subset C_{b},
$$

because $e\left(*, i^{b}\right) C_{b} \simeq E_{i}^{*(b)} C_{b} \otimes L\left(i^{b}\right)$ as an $R\left(b\left(\beta-\alpha_{i}\right)\right) \otimes R\left(b \alpha_{i}\right)$-module. Then, we have an epimorphism

$$
T \circ L(i)^{\circ b} \rightarrow C_{b}
$$

given by $v \otimes\langle i\rangle^{\circ b} \mapsto v$.

Note that $K_{\ell}$ is generated (as an $R\left(\ell \beta-\alpha_{i}\right)$-module) by $C_{a} \circ K_{b} \subset K_{\ell}$ and the elements $\left(e\left(a \beta-\alpha_{i}\right) \otimes H\right)(u \otimes v)$ with $u \in e(*, i) C_{a}$ and $v \in T$. Here $H=\tau_{b m} \cdots \tau_{1} e(i, b \beta)$ $\in R\left(b \beta+\alpha_{i}\right)$. Note that $e\left(a \beta-\alpha_{i}\right) \otimes H \in R(\ell \beta)$. Then $g: K_{\ell} \rightarrow q^{-b\left(\beta, \alpha_{i}\right)} K_{a} \circ C_{b}$ is given by

$$
g\left(C_{a} \circ K_{b}\right)=0 \text { and } g\left(\left(e\left(a \beta-\alpha_{i}\right) \otimes H\right)(u \otimes v)\right)=u \otimes v .
$$

Let $F:=\varepsilon_{\ell} \circ z^{b}$ and $G:=\varepsilon_{a} \circ R_{C_{b}}\left(L_{a}\right) \circ g$ be the two compositions $q_{i}^{2 b} K_{\ell} \circ L_{a} \rightarrow C_{\ell}$ appearing in (4.16). Then it is easy to see that $C_{a} \circ K_{b} \circ L_{a} \subset K_{\ell} \circ L_{a}$ is sent to zero by $G$, and $F\left(C_{a} \circ K_{b} \circ L_{a}\right)=0$ follows from $x_{b m}^{b} e(*, i) C_{b}=0$.

Hence in order to see the commutativity of (4.16), it is enough to show that $F$ and $G$ send $w:=\left(e\left(a \beta-\alpha_{i}\right) \otimes H\right)(u \otimes v) \otimes\langle i\rangle_{a}$ to the same element in $C_{\ell}$ up to a constant multiple.

Let us first calculate $F(w)$. We have

$$
\begin{aligned}
F(w) & =\varepsilon_{\ell}\left(\left(e\left(a \beta-\alpha_{i}\right) \otimes H\right)(u \otimes v) \otimes z^{b}\langle i\rangle_{\ell}\right) \\
& =x_{\ell m}^{b}\left(e\left(a \beta-\alpha_{i}\right) \otimes H\right) \varepsilon_{\ell}\left((u \otimes v) \otimes\langle i\rangle_{\ell}\right) \\
& =\left(e\left(a \beta-\alpha_{i}\right) \otimes x_{b m+1}^{b}\right)\left(e\left(a \beta-\alpha_{i}\right) \otimes H\right)(u \otimes v) \\
& =\left(e\left(a \beta-\alpha_{i}\right) \otimes\left(x_{b m+1}^{b} \tau_{b m} \cdots \tau_{1}\right)\right)(u \otimes v) .
\end{aligned}
$$


Now, we shall calculate $G(w)$, which is given by

$$
G(w)=\varepsilon_{a}\left(u \otimes R_{C_{b}}\left(L_{a}\right)\left(v \otimes\langle i\rangle_{a}\right)\right) .
$$

Lemma 4.14. For $v \in T$, we have (up to a constant multiple)

$$
R_{C_{b}}(\widetilde{L}(i))\left(v \otimes\langle i\rangle_{z}\right)=x_{b m+1}^{b} \tau_{b m} \cdots \tau_{1}\left(\langle i\rangle_{z} \otimes v\right) .
$$

Proof. Since $\left(C, R_{C}, \phi\right)$ is non-degenerate, we have $R_{C}(\widetilde{L}(i))=\mathrm{R}_{C, \widetilde{L}(i)}^{\text {norm }}$ up to a constant multiple. Since $\Lambda\left(C_{b}, L(i)\right)=\Lambda(T, L(i))+\Lambda\left(L(i)^{\circ b}, L(i)\right)$, the epimorphism $T \circ L(i)^{\circ b} \rightarrow C_{b}$ implies that the diagram

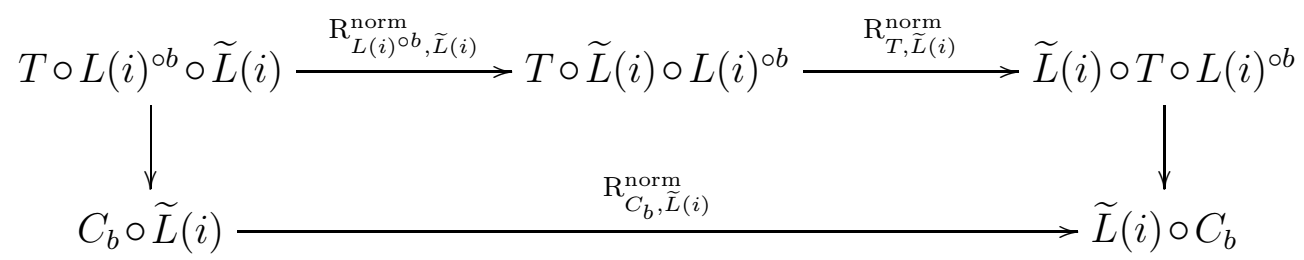

commutes. We have

$$
\mathrm{R}_{L(i)^{\circ b}, \widetilde{L}(i)}^{\mathrm{norm}}\left(\langle i\rangle^{\circ b} \bigotimes\langle i\rangle_{z}\right)=\mathrm{R}_{L(i)^{\circ b}, \widetilde{L}(i)}\left(\langle i\rangle^{\circ b} \otimes\langle i\rangle_{z}\right)=\varphi_{b} \cdots \varphi_{1}\left(\langle i\rangle_{z} \bigotimes\langle i\rangle^{\circ b}\right) .
$$

Now we shall show

$$
\varphi_{b} \cdots \varphi_{1}\left(\langle i\rangle_{z} \otimes\langle i\rangle^{\circ b}\right)=x_{b+1}^{b} \tau_{b} \cdots \tau_{1}\left(\langle i\rangle_{z} \otimes\langle i\rangle^{\circ b}\right)
$$

by induction on $b$. Assuming for $b-1$, we have

$$
\begin{aligned}
\varphi_{b} \cdots \varphi_{1}\left(\langle i\rangle_{z} \otimes\langle i\rangle^{\circ b}\right) & =\varphi_{b} x_{b}^{b-1} \tau_{b-1} \cdots \tau_{1}\left(\langle i\rangle_{z} \otimes\langle i\rangle^{\circ b}\right) \\
& =x_{b+1}^{b-1} \varphi_{b} \tau_{b-1} \cdots \tau_{1}\left(\langle i\rangle_{z} \otimes\langle i\rangle^{\circ b}\right) \\
& =x_{b+1}^{b-1}\left(x_{b+1} \tau_{b}-\tau_{b} x_{b+1}\right) \tau_{b-1} \cdots \tau_{1}\left(\langle i\rangle_{z} \otimes\langle i\rangle^{\circ b}\right) \\
& =x_{b+1}^{b} \tau_{b} \tau_{b-1} \cdots \tau_{1}\left(\langle i\rangle_{z} \otimes\langle i\rangle^{\circ b}\right) .
\end{aligned}
$$

Thus the induction proceeds, and we have obtained (4.20).

Hence we obtained

$$
\mathrm{R}_{L(i)^{\circ b}, \widetilde{L}(i)}^{\mathrm{norm}}\left(\langle i\rangle^{\circ b} \otimes\langle i\rangle_{z}\right)=x_{b+1}^{b} \tau_{b} \tau_{b-1} \cdots \tau_{1}\left(\langle i\rangle_{z} \otimes\langle i\rangle^{\circ b}\right) .
$$

On the other hand, since $\alpha_{i} \notin W^{*}(T)$, we have by [15, Proposition 2.12]

$$
\mathrm{R}_{T, \widetilde{L}(i)}^{\mathrm{norm}}\left(y \otimes\langle i\rangle_{z}\right)=\tau_{b(m-1)} \cdots \tau_{1}\left(\langle i\rangle_{z} \otimes y\right)
$$


for $y \in T$. Hence we have

$$
\begin{aligned}
& \left(\mathrm{R}_{T, \widetilde{L}(i)}^{\text {norm }} \circ L(i)^{\circ b}\right) \circ\left(T \circ \mathrm{R}_{L(i)^{\circ b}, \widetilde{L}(i)}^{\text {norm }}\right)\left(y \otimes\langle i\rangle^{\circ b} \otimes\langle i\rangle_{z}\right) \\
& \quad=\left(\mathrm{R}_{T, \widetilde{L}(i)}^{\text {norm }} \circ L(i)^{\circ b}\right)\left(y \otimes\left(x_{b+1}^{b} \tau_{b} \cdots \tau_{1}\left(\langle i\rangle_{z} \otimes\langle i\rangle^{\circ b}\right)\right)\right) \\
& \quad=x_{b m+1}^{b} \tau_{b m} \cdots \tau_{b m-b+1}\left(\left(\mathrm{R}_{T, \widetilde{L}(i)}^{\text {norm }}\left(y \otimes\langle i\rangle_{z}\right)\right) \otimes\langle i\rangle^{\circ b}\right) \\
& \quad=x_{b m+1}^{b} \tau_{b m} \cdots \tau_{1}\left(\langle i\rangle_{z} \otimes y \otimes\langle i\rangle^{\circ b}\right) .
\end{aligned}
$$

Together with the commutativity of diagram (4.19), we obtain the desired result.

Now, we resume the calculation of $G(w)$. By the lemma above, we obtain (up to a constant multiple)

$$
\begin{aligned}
G(w) & =\varepsilon_{a}\left(u \otimes\left(x_{b m+1}^{b} \tau_{b m} \cdots \tau_{1}\right)\left(\langle i\rangle_{a} \otimes v\right)\right) \\
& =\left(e\left(a \beta-\alpha_{i}\right) \otimes\left(x_{b m+1}^{b} \tau_{b m} \cdots \tau_{1}\right)\right)\left(\left(\varepsilon_{a}\left(u \otimes\langle i\rangle_{a}\right)\right) \otimes v\right) \\
& =\left(e\left(a \beta-\alpha_{i}\right) \otimes\left(x_{b m+1}^{b} \tau_{b m} \cdots \tau_{1}\right)\right)(u \otimes v) .
\end{aligned}
$$

Comparing it with (4.17), we obtain $F(w)=G(w)$ up to a constant multiple. Thus the induction proceeds, and we conclude that $\left(\widetilde{\varepsilon}_{\ell}, \widetilde{\eta}_{\ell}\right)$ is a quasi-adjunction for any $\ell$. It completes the proof of Theorem 4.12.

\section{LOCALiZATION OF $\mathscr{C}_{w}$}

We fix an element $w \in \mathbf{W}$ throughout this section.

5.1. Graded braiders associated with a Weyl group element. For $\Lambda \in \mathrm{P}_{+}$, we define

$$
\mathrm{C}_{w, \Lambda}:=\mathrm{M}(w \Lambda, \Lambda) .
$$

If no confusion arises, we simply write $C_{\Lambda}$ for $C_{w, \Lambda}$. For $i \in I$, we simply set

$$
\mathrm{C}_{i}:=\mathrm{C}_{\Lambda_{i}}
$$

Note that, for $\Lambda=\sum_{k=1}^{n} \Lambda_{i_{k}}, \mathrm{C}_{\Lambda}$ is a self-dual real simple $R$-module which is isomorphic to $C_{\Lambda_{i_{1}}} \circ C_{\Lambda_{i_{2}}} \circ \cdots \circ C_{\Lambda_{i_{n}}}$ up to a grading shift [15, Proposition 4.2]. We shall show that $\mathrm{C}_{i}$ is a real graded braider in the category $R$-gmod with the non-degenerate graded braider structure.

For $i \in I$, we set

$$
\lambda_{i}:= \begin{cases}w \Lambda_{i}+\Lambda_{i} & \text { if } w \Lambda_{i} \neq \Lambda_{i} \\ 0 & \text { if } w \Lambda_{i}=\Lambda_{i}\end{cases}
$$


Note that $\mathrm{C}_{i} \simeq \mathbf{1}$ if and only if $w \Lambda_{i}=\Lambda_{i}$. By Lemma 3.15, we have

$$
\Lambda\left(\mathrm{C}_{i}, L(j)\right)=\left(\alpha_{j}, \alpha_{j}\right) \varepsilon_{j}^{*}\left(\mathrm{C}_{i}\right)+\left(\alpha_{j}, w \Lambda_{i}-\Lambda_{i}\right)=\left(\lambda_{i}, \alpha_{j}\right) .
$$

Applying Lemma 4.3, we have the non-degenerate graded braider $\left(\mathrm{C}_{i}, R_{\mathrm{C}_{i}}, \phi_{i}\right)$ for $i \in I$.

Proposition 5.1. The family $\left(\mathrm{C}_{i}, R_{\mathrm{C}_{i}}, \phi_{i}\right)_{i \in I}$ is a real commuting family of graded braiders in $R$-gmod with the non-degenerate graded braider structure and

$$
\phi_{i}(\beta)=-\left(\lambda_{i}, \beta\right) \quad \text { for any } \beta \in \mathbf{Q} \text {. }
$$

Proof. The reality follows from Lemma 4.6 and $\left(\lambda_{i}, \operatorname{wt}\left(\mathrm{C}_{j}\right)\right)+\left(\lambda_{j}, \operatorname{wt}\left(\mathrm{C}_{i}\right)\right)=0$.

Theorem 5.2. For $i \in I$ and any $R(\beta)$-module $N$ in $\mathscr{C}_{w}, R_{\mathrm{C}_{i}}(N)$ is an isomorphism.

Proof. We take a reduced expression $\underline{w}=s_{i_{1}} \cdots s_{i_{\ell}}$ of $w$, and set $w_{0}=\mathrm{id}, w_{k}=$ $s_{i_{1}} \cdots s_{i_{k}}, \beta_{k}:=w_{k-1} \alpha_{i_{k}}$ and $\mathrm{S}_{k}:=\mathrm{M}\left(w_{k} \Lambda_{i_{k}}, w_{k-1} \Lambda_{i_{k}}\right)$ for $k=1, \ldots, \ell$. Then $\mathrm{S}_{k}$ is the cuspidal $R\left(\beta_{k}\right)$-module corresponding to the positive root $\beta_{k}$ by Proposition 3.24. Setting $\lambda=\Lambda_{i}, \mu=\Lambda_{i_{k}}, s^{\prime}=w_{k}, s=w_{k} w, t^{\prime}=$ id and $t=w_{k-1}$ in Proposition 3.27, we have that $C_{i}$ and $S_{k}$ strongly commute and

$$
\Lambda\left(C_{i}, \mathrm{~S}_{k}\right)=\left(\lambda_{i}, \beta_{k}\right)=-\phi_{i}\left(\beta_{k}\right) .
$$

Hence Proposition 4.4 implies that $R_{\mathrm{C}_{i}}\left(\mathrm{~S}_{k}\right)$ is an isomorphism. Let $\mathscr{C}$ be the full subcategory of $\mathscr{C}_{w}$ consisting of objects $M$ such that $R_{\mathrm{C}_{i}}(M)$ is an isomorphism. Then $\mathscr{C}$ has the following proprieties:

(a) $\mathscr{C}$ is stable by taking extensions, kernels, cokernels, and convolutions,

(b) $\mathscr{C}$ contains all the $S_{k}$ 's.

Since $\mathscr{C}_{w}$ is the smallest subcategory of $\mathscr{C}_{w}$ which has these properties, $\mathscr{C}=\mathscr{C}_{w}$.

We set $\Gamma:=\mathbb{Z}^{\oplus I}$ and define a $\mathbb{Z}$-bilinear map $\mathrm{H}: \Gamma \times \Gamma \rightarrow \mathbb{Z}$ by

$$
\mathrm{H}\left(e_{i}, e_{j}\right):=-\widetilde{\Lambda}\left(\mathrm{C}_{i}, \mathrm{C}_{j}\right)=\left(\Lambda_{i}, w \Lambda_{j}-\Lambda_{j}\right) .
$$

Then, for $i, j \in I$, we have

$$
\begin{aligned}
\phi_{i}\left(-\operatorname{wt}\left(C_{j}\right)\right) & =\left(w \Lambda_{i}+\Lambda_{i}, w \Lambda_{j}-\Lambda_{j}\right)=\left(\Lambda_{i}, w \Lambda_{j}\right)-\left(\Lambda_{j}, w \Lambda_{i}\right) \\
& =\mathrm{H}\left(e_{i}, e_{j}\right)-\mathrm{H}\left(e_{j}, e_{i}\right) .
\end{aligned}
$$

Thanks to Theorem 2.12 and Proposition 5.1, we have the localization of $\mathscr{C}_{w}$ by the non-degenerate graded braiders $C_{i}$, which we denote by

$$
\widetilde{\mathscr{C}}_{w}:=\mathscr{C}_{w}\left[C_{i}^{0-1} \mid i \in I\right] \text {. }
$$


By the choice above of $\mathrm{H}$, for $\alpha=\sum_{i \in I} m_{i} e_{i} \in \Gamma_{\geq 0}$, we have

$$
\mathrm{C}_{\Lambda} \simeq(\mathbf{1}, \alpha), \quad \mathrm{C}_{\Lambda}^{-1} \simeq q^{\mathrm{H}(\alpha, \alpha)}(\mathbf{1},-\alpha)
$$

where $\Lambda=\sum_{i \in I} m_{i} \Lambda_{i}$ by Lemma 3.14. Thus, for $\Lambda=\sum_{i \in I} a_{i} \Lambda_{i} \in \mathrm{P}$, we simply write $\mathrm{C}_{\Lambda}:=\left(\mathbf{1}, \sum_{i \in I} a_{i} e_{i}\right) \in \widetilde{\mathscr{C}}_{w}$.

Therefore, there exists a monoidal functor $\Phi: \mathscr{C}_{w} \rightarrow \widetilde{\mathscr{C}}_{w}$ such that

(i) the objects $\Phi\left(C_{i}\right)$ are invertible in $\widetilde{\mathscr{C}}_{w}$,

(ii) $\Phi\left(R_{\mathrm{C}_{\Lambda}}(X)\right)$ is an isomorphism for any $\Lambda \in \mathrm{P}_{+}$and $X \in \mathscr{C}_{w}$,

(iii) for any simple object $S$ of $\mathscr{C}_{w}$, the object $\Phi(S)$ is simple in $\widetilde{\mathscr{C}}_{w}$,

(iv) every simple object of $\widetilde{\mathscr{C}}_{w}$ is isomorphic to $\mathrm{C}_{\Lambda} \circ \Phi(S)$ for some simple object $S$ of $\mathscr{C}_{w}$ and $\Lambda \in \mathrm{P}$,

(v) for two simple objects $S$ and $S^{\prime}$ in $\mathscr{C}_{w}$ and $\Lambda, \Lambda^{\prime} \in \mathrm{P}, \mathrm{C}_{\Lambda} \circ \Phi(S) \simeq \mathrm{C}_{\Lambda^{\prime}} \circ \Phi\left(S^{\prime}\right)$ in $\widetilde{\mathscr{C}}_{w}$ if and only if $q^{\mathrm{H}(\Lambda, \mu)} \mathrm{C}_{\Lambda+\mu} \circ S \simeq q^{\mathrm{H}\left(\Lambda^{\prime}, \mu\right)} C_{\Lambda^{\prime}+\mu} \circ S^{\prime}$ in $\mathscr{C}_{w}$ for some $\mu \in \mathrm{P}$ such that $\Lambda+\mu, \Lambda^{\prime}+\mu \in \mathrm{P}_{+}$,

(vi) the category $\widetilde{\mathscr{C}}_{w}$ is abelian and every objects has finite length.

Here, (iii)-(vi) follow from Proposition 4.8. The grading shift functor $q$ and the contravariant functor $M \mapsto M^{\star}$ on $\mathscr{C}_{w}$ are extended to $\widetilde{\mathscr{C}}_{w}$. Moreover we have

$$
(M \circ N)^{\star} \simeq q^{(\operatorname{wt}(M), \mathrm{wt}(N))} N^{\star} \circ M^{\star} \text { for } M, N \in \widetilde{\mathscr{C}}_{w} .
$$

Since every simple object of $\widetilde{\mathscr{C}}_{w}$ is isomorphic to $C^{\alpha} \circ \Phi(S)$ for some simple module $S$ of $\mathscr{C}_{w}$, one can prove the following.

Lemma 5.3. For any simple module $M \in \widetilde{\mathscr{C}}_{w}$, there exists a unique $n \in \mathbb{Z}$ such that $q^{n} M$ is self-dual.

Corollary 5.4. The Grothendieck ring $K\left(\widetilde{\mathscr{C}}_{w}\right)$ of $\widetilde{\mathscr{C}}_{w}$ is isomorphic to the left ring of quotients of the ring $K\left(\mathscr{C}_{w}\right)$ with respect to the multiplicative subset

$$
S:=\left\{q^{k} \prod_{i \in I}\left[\mathrm{C}_{i}\right]^{a_{i}} \mid k \in \mathbb{Z},\left(a_{i}\right)_{i \in I} \in \mathbb{Z}_{\geq 0}^{I}\right\} .
$$

Proof. Note that the set $S$ is a left denominator set in $K\left(\mathscr{C}_{w}\right)$ since $\left[\mathrm{C}_{i}\right]$ is $q$-commuting with any class of simple object. Since $S$ is invertible in $K\left(\widetilde{\mathscr{C}}_{w}\right)$, we have an algebra map $S^{-1} K\left(\mathscr{C}_{w}\right) \rightarrow K\left(\widetilde{\mathscr{C}}_{w}\right)$. It is evidently surjective. In order to see injectivity, it is enough to show that the left multiplication on $K\left(\mathscr{C}_{w}\right)$ by $\left[\mathrm{C}_{i}\right]$ is injective. It is well known.

Remark 5.5. The $\operatorname{ring} \mathbb{Q}\left(q^{1 / 2}\right) \otimes_{\mathbb{Z}\left[q^{ \pm 1}\right]} K\left(\widetilde{\mathscr{C}}_{w}\right)$, which is a localization of the quantum cluster algebra $\mathbb{Q}\left(q^{1 / 2}\right) \otimes_{\mathbb{Z}\left[q^{ \pm 1}\right]} K\left(\mathscr{C}_{w}\right)$, is the subalgebra of the skew field of fractions $K$ of an initial quantum torus $\mathbb{Q}\left(q^{1 / 2}\right)\left[X_{i}^{ \pm 1} \mid i \in J\right]$ generated by all the cluster variables 
and the inverses of the frozen variables. Thus it is the quantum cluster algebra in the sense of [5].

5.2. Rank-one case. Let us briefly describe the localization $\widetilde{\mathscr{C}}_{w}$ without proof when $\mathfrak{g}$ is of rank one. Set $I=\{i\}$. Let $\widetilde{\mathscr{R}}$ be the localization of $R$-gmod by $L(i)$. Note that $R$-gmod is a symmetric monoidal category, since $\mathrm{R}_{M, N}$ (see Section 3.2) is always an isomorphism. We have the decomposition

$$
\widetilde{\mathscr{R}}=\bigoplus_{n \in \mathbb{Z}} \widetilde{\mathscr{R}}_{0} \circ L(i)^{\circ n} .
$$

Here $\widetilde{\mathscr{R}}_{0}$ is the full subcategory of $\widetilde{\mathscr{R}}$ consisting of objects of weight 0 . In another words, it is the full subcategory consisting of the objects of the form $M \circ L(i)^{\circ-n}$ for $n \in \mathbb{Z}_{>0}$ and $M \in R\left(n \alpha_{i}\right)$-gmod. The monoidal category $\widetilde{\mathscr{R}}_{0}$ is described as follows.

Let $\mathrm{S}$ be the ring of symmetric polynomials. Hence, $\mathrm{S}=\bigoplus_{d \in \mathbb{Z}_{\geq 0}} \mathrm{~S}_{d}$ is positively graded and

$$
\mathrm{S}_{d}=\lim _{n \in \mathbb{Z}_{>0}} \mathbf{k}\left[x_{1}, \ldots, x_{n}\right]_{d}^{\mathfrak{S}_{n}}
$$

where $\mathbf{k}\left[x_{1}, \ldots, x_{n}\right]_{d}^{\mathfrak{S}_{n}}$ is the space of symmetric polynomials of $n$ variables with degree d. Then

$$
\mathbf{S}=\mathbf{k}\left[e_{k} \mid k \in \mathbb{Z}_{>0}\right]
$$

where $e_{k}$ is the elementary symmetric polynomial of degree $k$. Let us take the generating function

$$
E(t)=\sum_{k=0}^{\infty} e_{k} t^{k}
$$

where $e_{0}=1$.

The ring $\mathbf{S}$ has a well known structure of (co-commutative) Hopf algebra by the coproduct defined by

$$
\Delta(E(t))=E(t) \otimes E(t) .
$$

Namely,

$$
\Delta\left(e_{n}\right)=\sum_{k=0}^{n} e_{k} \otimes e_{n-k} .
$$

Then its antipode $S$ is given by

$$
S(E(t))=E(t)^{-1}
$$

and its counit is given by

$$
\varepsilon(E(t))=1
$$


Let us denote by S-gmod the category of graded S-modules which are finite-dimensional over k. Then, S-gmod has a structure of monoidal category. Then, $\widetilde{\mathscr{R}}_{0}$ is equivalent to the category S-gmod as a monoidal category.

By this correspondence, the full subcategory $R\left(n \alpha_{i}\right) \operatorname{gmod} \otimes L(i)^{\circ-n} \subset \widetilde{\mathscr{R}}_{0}$ is precisely equivalent to the full subcategory of S-gmod consisting of modules $M$ such that $\left.e_{k}\right|_{M}=0$ for $k>n$, namely $\mathbf{k}\left[x_{1}, \ldots, x_{n}\right]^{\mathfrak{S}_{n}}$-gmod. This equivalence is obtained by the Morita equivalence through $\mathbf{k}\left[x_{1}, \ldots, x_{n}\right]^{\mathfrak{S}_{n}} \simeq \operatorname{END}_{R\left(n \alpha_{i}\right)}\left(P\left(i^{n}\right)\right)$. Here $P\left(i^{n}\right)$ is a unique indecomposable projective $R\left(n \alpha_{i}\right)$-module.

\subsection{Left rigidity of $\widetilde{\mathscr{C}}_{w}$.}

First note the following lemma which immediately follows from Proposition 4.11.

Lemma 5.6. Let $M$ be a simple module in $\mathscr{C}_{w}$. Then $M$ has a right dual (respectively, left dual) in $\widetilde{\mathscr{C}}_{w}$ if and only if there exists a surjective homomorphism $M \circ X \rightarrow \mathrm{C}_{\Lambda}$ (respectively, $X \circ M \rightarrow \mathrm{C}_{\Lambda}$ ) for some module $X$ in $\mathscr{C}_{w}$ and for some $\Lambda \in \mathrm{P}_{+}$.

We now consider duals of objects in the localization $\widetilde{\mathscr{C}}_{w}$. For an object $M$ in $\widetilde{\mathscr{C}}_{w}$, we denote by $\mathscr{D}(M)$ the right dual of $M$ and by $\mathscr{D}^{-1}(M)$ the left dual of $M$, if they exist.

Theorem 5.7. Every simple object $M$ in $\widetilde{\mathscr{C}}_{w}$ has a right dual.

Proof. For simplicity, we ignore the grading in the course of the proof. We will proceed by induction on $l=\ell(w)$. We take a reduced expression $\underline{w}=s_{i_{1}} \cdots s_{i_{l}}$ of $w$, and set $w_{0}=$ id and $w_{<k+1}=w_{\leq k}=s_{i_{1}} \cdots s_{i_{k}}$. Note that the full subcategory consisting of objects in $\mathscr{C}_{w}$ which have right duals in $\tilde{\mathscr{C}}_{w}$ is closed by taking the kernels, the cokernels and the convolution products. Note also that any simple object is contained in the smallest full subcategory of $\mathscr{C}_{w}$ which is closed by taking the kernels, the cokernels and the convolution products and contains all the cuspidal modules $\mathrm{S}_{k}:=\mathrm{M}\left(w_{\leq k} \Lambda_{i_{k}}, w_{<k} \Lambda_{i_{k}}\right)$ $(1 \leq k \leq l)$. Hence it is enough to show that every $\mathrm{S}_{k}$ has a right dual.

For this, we shall show that, for any $k$, we can find an epimorphism $\mathrm{S}_{k} \circ X \rightarrow \mathrm{C}_{\Lambda}$ for some $X \in \mathscr{C}_{w}$ and some $\Lambda \in \mathrm{P}_{+}$. We argue by induction on $l$.

Since there exists a surjective homomorphism

$$
\mathrm{S}_{l} \circ \mathrm{M}\left(w_{<l} \Lambda_{i_{l}}, \Lambda_{i_{l}}\right) \rightarrow \mathrm{M}\left(w \Lambda_{i_{l}}, \Lambda_{i_{l}}\right)=\mathrm{C}_{i_{l}}
$$

by $[15$, Proposition 4.6$]$, the object $S_{l}$ has a right dual in $\widetilde{\mathscr{C}}_{w}$.

Now assume that $k<l$, and set $w^{\prime}:=w_{<l}$. Note that $\mathrm{S}_{k} \in \mathscr{C}_{w^{\prime}}$. By the induction hypothesis, there exist a simple module $X \in \mathscr{C}_{w^{\prime}}, a_{i} \in \mathbb{Z}_{\geq 0}(i \in I)$ and a surjective homomorphism

$$
\mathrm{S}_{k} \circ X \rightarrow \mathrm{O}_{i \in I} \mathrm{M}\left(w_{<l} \Lambda_{i}, \Lambda_{i}\right)^{\circ a_{i}}
$$

Hence it is enough to show that $\mathrm{M}\left(w_{<l} \Lambda_{i}, \Lambda_{i}\right)$ has a right dual in $\widetilde{\mathscr{C}}_{w}$ for all $i \in I$. 
If $i \neq i_{l}$, then $\mathrm{M}\left(w_{<l} \Lambda_{i}, \Lambda_{i}\right)=\mathrm{M}\left(w \Lambda_{i}, \Lambda_{i}\right)=\mathrm{C}_{i}$.

Suppose that $i=i_{l}$. By the T-system ([14, Proposition 10.2.5 (i)]), we have

$$
\mathrm{M}\left(w_{<l} \Lambda_{i_{l}}, \Lambda_{i_{l}}\right) \circ \mathrm{M}\left(w \Lambda_{i_{l}}, s_{i_{l}} \Lambda_{i_{l}}\right) \rightarrow \mathrm{O}_{j \neq i_{l}} \mathrm{M}\left(w \Lambda_{j}, \Lambda_{j}\right)^{\mathrm{O}-a_{j, i_{l}}} .
$$

Note that the T-system ([9, Proposition 3.2], [14, Proposition 10.2.5 (i)]) also holds for non-symmetric case if $q$ is replaced by $q_{i}$. Hence $\mathrm{M}\left(w_{<l} \Lambda_{i_{l}}, \Lambda_{i_{l}}\right)$ has a right dual.

We shall show that any object in $\widetilde{\mathscr{C}}_{w}$ has a left dual. By Proposition 5.1, there exists a localization of $R$-gmod by the non-degenerate graded braiders $C_{i}$, which is denoted by $\widetilde{\mathscr{R}}$; i.e.,

$$
\widetilde{\mathscr{R}}:=R-\operatorname{gmod}\left[\mathrm{C}_{i}^{\circ-1} \mid i \in I\right] .
$$

Since $\mathscr{C}_{w}$ is a full subcategory of $R$-gmod, the canonical embedding $\mathscr{C}_{w} \longmapsto R$-gmod induces the fully faithful monoidal functor

$$
\imath: \widetilde{\mathscr{C}}_{w} \longmapsto \widetilde{\mathscr{R}}
$$

by the construction of localization. Moreover, the essential image is stable under taking subquotients, extensions and grading shifts by (a graded version of) Proposition 2.10.

Set $I_{w}:=\left\{i \in I \mid w \Lambda_{i} \neq \Lambda_{i}\right\}$. Note that $I_{w}=\left\{i_{1}, \ldots, i_{l}\right\}$ for any reduced expression $\underline{w}=s_{i_{1}} \cdots s_{i_{l}}$ of $w$.

Theorem 5.8. Assume that $I=I_{w}$.

(i) Every object in $\widetilde{\mathscr{R}}$ has a left dual.

(ii) Moreover, the left dual of any object in $\widetilde{\mathscr{R}}$ belongs to $\widetilde{\mathscr{C}}_{w}$.

Proof. By Theorem 4.12 and $\varepsilon_{i}^{*}\left(\mathrm{C}_{i}\right)=1, L_{\ell}(i)$ has a left dual $\left(\mathrm{C}_{i}^{\circ \ell}\right)^{\circ-1} \circ E_{i}^{*}\left(\mathrm{C}_{i}^{\circ \ell}\right)$ in $\widetilde{\mathscr{R}}$ for any $i \in I$ and $\ell \in \mathbb{Z}_{>0}$. Since $E_{i}^{*}\left(C_{i}^{\circ}\right)$ belongs to $\mathscr{C}_{w}, \mathscr{D}^{-1}\left(L_{\ell}(i)\right)$ belongs to $\widetilde{\mathscr{C}}_{w}$.

It suffices to show that any finite-dimensional $R$-module has a left dual which is contained in $\widetilde{\mathscr{C}}_{w}$. Let $Q$ be a nonzero finite-dimensional $R$-module. Since the generators $x_{i}$ act on any finite-dimensional $R$-module nilpotently, there exist $R$-modules $P_{1}$ and $P_{2}$ which are direct sums of modules of the form $L_{\ell_{1}}\left(\nu_{1}\right) \circ L_{\ell_{2}}\left(\nu_{2}\right) \circ \cdots \circ L_{\ell_{t}}\left(\nu_{t}\right)$ such that

$$
P_{1} \stackrel{\phi}{\longrightarrow} P_{2} \longrightarrow Q \longrightarrow 0
$$

is exact. Since objects of the form $L_{\ell_{1}}\left(\nu_{1}\right) \circ L_{\ell_{2}}\left(\nu_{2}\right) \circ \cdots \circ L_{\ell_{t}}\left(\nu_{t}\right)$ have left duals in $\widetilde{\mathscr{C}}_{w}, P_{1}$ and $P_{2}$ have left duals $\mathscr{D}^{-1}\left(P_{1}\right)$ and $\mathscr{D}^{-1}\left(P_{2}\right)$ respectively in $\widetilde{\mathscr{C}}_{w}$. Thus, the cokernel $Q$ also has a left dual in $\widetilde{\mathscr{C}}_{w}$, which is isomorphic to the kernel of the morphism $\mathscr{D}^{-1}(\phi): \mathscr{D}^{-1}\left(P_{2}\right) \rightarrow \mathscr{D}^{-1}\left(P_{1}\right)$.

Theorem 5.9. Assume that $I=I_{w}$. The functor $\imath: \widetilde{\mathscr{C}}_{w} \longrightarrow \widetilde{\mathscr{R}}$ is an equivalence of categories. 
Proof. Let $S$ be a simple object in $\widetilde{R}$. By Theorem 5.8 , the left dual $\mathscr{D}^{-1}(S)$ belongs to $\widetilde{\mathscr{C}}_{w}$. Then $\mathscr{D}^{-1}(S)$ does not vanish. Let us take a simple quotient $f: \mathscr{D}^{-1}(S) \rightarrow$ $M$ of $\mathscr{D}^{-1}(S)$. Then $M$ has a right dual $\mathscr{D}(M)$ in $\tilde{\mathscr{C}}_{w}$ and we have a morphism $\mathscr{D}(f): \mathscr{D}(M) \rightarrow S$. Let us show that $\mathscr{D}(f)$ is a monomorphism. Let $N=\operatorname{ker}(\mathscr{D}(f))$. Then we have an exact sequence

$$
0 \rightarrow N \stackrel{\psi}{\longrightarrow} \mathscr{D}(M) \rightarrow S
$$

Taking its left dual, we obtain

$$
\mathscr{D}^{-1}(S) \rightarrow M \stackrel{\mathscr{D}^{-1}(\psi)}{\longrightarrow} \mathscr{D}^{-1}(N) .
$$

Since the composition vanishes, we obtain $\mathscr{D}^{-1}(\psi)=0$. Hence $\psi=0$. Thus we conclude that $\mathscr{D}(f)$ is a monomorphism.

Since $S$ is simple, $\mathscr{D}(f)$ is an isomorphism. Hence $\mathscr{D}(M) \simeq S$, and hence $S \in \widetilde{\mathscr{C}}_{w}$.

Thus, we have proved that any simple object of $\widetilde{\mathscr{R}}$ belongs to $\widetilde{\mathscr{C}}_{w}$. Since $\widetilde{\mathscr{C}}_{w}$ is stable by extension, we conclude that $\widetilde{\mathscr{C}}_{w} \simeq \widetilde{\mathscr{R}}$.

Corollary 5.10. Assume that $I=I_{w}$. Then $X \in R$-gmod belongs to $\mathscr{C}_{w}$ if and only if $R_{\mathrm{C}_{i}}(X)$ is an isomorphism for any $i \in I$.

Proof. Assume that $R_{\mathrm{C}_{i}}(X)$ is an isomorphism for any $i \in I$. Let us show that $X \in \mathscr{C}_{w}$.

Since it suffices that any simple subquotient of $X$ belongs to $\mathscr{C}_{w}$, we may assume that $X$ is simple from the beginning (see Proposition 4.9 (i)). There exists $Z \in \mathscr{C}_{w}$ and $\Lambda \in \mathrm{P}_{+}$such that $X \simeq \mathrm{C}_{-\Lambda} \circ Z$ in $(R \text {-gmod })^{\sim}$. We may assume that $Z$ is simple. Then $Z \simeq \mathrm{C}_{\Lambda} \circ X$ by Proposition 4.8 (iii). Hence we have $\mathrm{W}(X) \subset \mathrm{W}(Z) \subset \mathrm{Q}_{+} \cap w \mathrm{Q}_{-}$ by [15, Lemma 2.2].

Since $-\operatorname{wt}(M) \in \sum_{i \in I_{w}} \mathbb{Z}_{\geq 0} \alpha_{i}$ for any module $M \in \mathscr{C}_{w}$, letting $R^{\prime}$ be the quiver Hecke algebra associated with the Cartan matrix $\mathrm{A}^{\prime}=\left(\left\langle h_{i}, \alpha_{j}\right\rangle\right)_{i, j \in I_{w}}$, we regard $\mathscr{C}_{w}$ as a subcategory of $R^{\prime}$-gmod. Then Theorem 5.8 and Theorem 5.9 give the following.

Corollary 5.11. The category $\widetilde{\mathscr{C}}_{w}$ is left rigid, i.e., every object of $\widetilde{\mathscr{C}}_{w}$ has a left dual in $\tilde{\mathscr{C}}_{w}$.

The following conjecture arises naturally from Corollary 5.11.

Conjecture 5.12. The category $\widetilde{\mathscr{C}}_{w}$ is rigid, i.e., every object of $\widetilde{\mathscr{C}}_{w}$ has left and right duals.

In the case of the longest element $w_{0} \in \mathrm{W}$, Conjecture 5.12 is true. 
Theorem 5.13. We assume that the quiver Hecke algebra $R$ is of finite type. Let $w_{0}$ be the longest element of $\mathrm{W}$. Then the category $\widetilde{\mathscr{C}}_{w_{0}}$ is rigid.

Proof. Note that $\mathscr{C}_{w_{0}}=R$-gmod in this case. Thanks to Corollary 5.11, it suffices to show that every object has a right dual.

For $\beta \in \mathrm{Q}_{+}$with $m=\operatorname{ht}(\beta)$, we have the automorphism of $R(\beta)$ defined by

$$
\begin{aligned}
e\left(\nu_{1}, \ldots, \nu_{m}\right) & \mapsto e\left(\nu_{m}, \ldots, \nu_{1}\right), \\
x_{i} e\left(\nu_{1}, \ldots, \nu_{m}\right) & \mapsto x_{m-i+1} e\left(\nu_{m}, \ldots, \nu_{1}\right), \\
\tau_{j} e\left(\nu_{1}, \ldots, \nu_{m}\right) & \mapsto-\tau_{m-j} e\left(\nu_{m}, \ldots, \nu_{1}\right),
\end{aligned}
$$

which induces a covariant functor $\mathfrak{a}: R$-gmod $\rightarrow R$-gmod such that $\mathfrak{a}^{2} \simeq$ id and $\mathfrak{a}(M \circ N) \simeq \mathfrak{a}(N) \circ \mathfrak{a}(M)$. For $i \in I$, let $i^{*}$ be an index such that $\alpha_{i^{*}}=-w_{0} \alpha_{i}$. Set $\beta_{i}=\Lambda_{i}-w_{0} \Lambda_{i}$. Then module $C_{i}$ is a unique (up to an isomorphism) simple $R\left(\beta_{i}\right)$-module satisfying the condition

$$
\varepsilon_{j}^{*}\left(\mathrm{C}_{i}\right)=\delta_{j, i} \quad \text { for any } j \in I .
$$

Since $\varepsilon_{j}^{*}\left(\mathfrak{a}\left(\mathrm{C}_{i}\right)\right)=\varepsilon_{j}\left(\mathrm{C}_{i}\right)=\delta_{j, i^{*}}$, and $\beta_{i^{*}}=\beta_{i}$, we obtain

$$
\mathfrak{a}\left(\mathrm{C}_{i}\right) \simeq \mathrm{C}_{i^{*}} \text { for } i \in I .
$$

Hence the functor $\mathfrak{a}$ induces the functor $\tilde{\mathfrak{a}}: \widetilde{\mathscr{C}}_{w_{0}} \rightarrow \widetilde{\mathscr{C}}_{w_{0}}$ which satisfies

$$
\tilde{\mathfrak{a}}^{2} \simeq \text { id } \quad \text { and } \quad \tilde{\mathfrak{a}}(X \circ Y) \simeq \tilde{\mathfrak{a}}(Y) \circ \tilde{\mathfrak{a}}(X) \quad \text { for } X, Y \in \widetilde{\mathscr{C}}_{w_{0}} .
$$

It is now straightforward to check that $\tilde{\mathfrak{a}}\left(\mathscr{D}^{-1}(\tilde{\mathfrak{a}}(X))\right)$ is a right dual of $X$.

Proposition 5.14. Assume the conditions and notations in Theorem 5.13. Then, we have isomorphisms (ignoring the grading shifts)

$$
\left(\mathscr{D}^{-1}\right)^{3}(L(i)) \simeq L\left(i^{*}\right) \circ C_{-\alpha_{i}} \text { for any } i \in I,
$$

and

$$
\left(\mathscr{D}^{-1}\right)^{6}(M) \simeq M \circ \mathrm{C}_{\beta+w_{0} \beta} \quad \text { for any simple } R(\beta) \text {-module } M .
$$

Proof. In the course of the proof, we ignore grading shifts. Since we have an epimorphism

$$
\mathrm{M}\left(w_{0} \Lambda_{i}, s_{i} \Lambda_{i}\right) \circ L(i) \rightarrow \mathrm{C}_{i}
$$

we have

$$
\mathscr{D}^{-1}(L(i)) \simeq \mathrm{M}\left(w_{0} \Lambda_{i}, s_{i} \Lambda_{i}\right) \circ \mathrm{C}_{i}^{\circ-1} .
$$

Now by T-system ([14, Proposition 10.2.5]), we have an epimorphism

$$
\mathrm{M}\left(w_{0} s_{i} \Lambda_{i}, \Lambda_{i}\right) \circ \mathrm{M}\left(w_{0} \Lambda_{i}, s_{i} \Lambda_{i}\right) \rightarrow \mathrm{M}\left(w_{0} \lambda, \lambda\right) .
$$


Here $\lambda=s_{i} \Lambda_{i}+\Lambda_{i}$. Hence we have

$$
\mathscr{D}^{-1}\left(\mathrm{M}\left(w_{0} \Lambda_{i}, s_{i} \Lambda_{i}\right)\right) \simeq \mathrm{M}\left(w_{0} s_{i} \Lambda_{i}, \Lambda_{i}\right) \circ \mathrm{C}_{-\lambda} .
$$

Finally, the epimorphism

$$
\mathrm{M}\left(w_{0} \Lambda_{i}, w_{0} s_{i} \Lambda_{i}\right) \circ \mathrm{M}\left(w_{0} s_{i} \Lambda_{i}, \Lambda_{i}\right) \rightarrow \mathrm{M}\left(w_{0} \Lambda_{i}, \Lambda_{i}\right)
$$

gives

$$
\mathscr{D}^{-1}\left(\mathrm{M}\left(w_{0} s_{i} \Lambda_{i}, \Lambda_{i}\right)\right) \simeq L\left(i^{*}\right) \circ \mathrm{C}_{-\Lambda_{i}} .
$$

Indeed, $\mathrm{M}\left(w_{0} \Lambda_{i}, w_{0} s_{i} \Lambda_{i}\right) \simeq L\left(i^{*}\right)$ because $w_{0} s_{i} \Lambda_{i}-w_{0} \Lambda_{i}=\alpha_{i^{*}}$.

Thus we obtain

$$
\begin{aligned}
\left(\mathscr{D}^{-1}\right)^{3}(L(i)) & \simeq\left(\mathscr{D}^{-1}\right)^{2}\left(\mathrm{M}\left(w_{0} \Lambda_{i}, s_{i} \Lambda_{i}\right) \circ \mathrm{C}_{-\Lambda_{i}}\right) \\
& \simeq\left(\mathscr{D}^{-1}\right)\left(\mathrm{M}\left(w_{0} s_{i} \Lambda_{i}, \Lambda_{i}\right) \circ \mathrm{C}_{-\lambda}\right) \circ \mathrm{C}_{-\Lambda_{i}} \\
& \simeq L\left(i^{*}\right) \circ \mathrm{C}_{-\Lambda_{i}} \circ \mathrm{C}_{\lambda} \circ \mathrm{C}_{-\Lambda_{i}} \\
& \simeq L\left(i^{*}\right) \circ \mathrm{C}_{-\alpha_{i}} .
\end{aligned}
$$

Then one has

$$
\begin{aligned}
\left(\mathscr{D}^{-1}\right)^{6}(L(i)) & \simeq\left(\mathscr{D}^{-1}\right)^{-3}\left(L\left(i^{*}\right) \circ \mathrm{C}_{-\alpha_{i}}\right) \\
& \simeq L(i) \circ \mathrm{C}_{-\alpha_{i^{*}}} \circ \mathrm{C}_{\alpha_{i}} \\
& \simeq L(i) \circ \mathrm{C}_{w_{0} \alpha_{i}+\alpha_{i}} .
\end{aligned}
$$

Hence we have obtained (5.2) for $\beta \in \mathrm{Q}_{+}$such that $\mathrm{ht}(\beta)=1$. Let us show (5.2) by induction on $\operatorname{ht}(\beta)$. Assume that $\operatorname{ht}(\beta)>1$. Let $M$ be a simple $R(\beta)$-module. Take $i \in I$ such that $\varepsilon_{i}(M)>0$, and set $M_{0}=\widetilde{E}_{i}(M)$. Then we have $M \simeq L(i) \nabla M_{0}$. Hence $M$ is the image of a non-zero homomorphism $f: L(i) \circ M_{0} \rightarrow M_{0} \circ L(i)$. Note that such an $f$ is unique up to a constant multiple. Applying the exact functor $\left(\mathscr{D}^{-1}\right)^{6}$, we conclude that $\left(\mathscr{D}^{-1}\right)^{6}(M)$ is the image of a non-zero morphism

$$
\left(\mathscr{D}^{-1}\right)^{6}(f):\left(\mathscr{D}^{-1}\right)^{6}\left(M_{0}\right) \circ\left(\mathscr{D}^{-1}\right)^{6}(L(i)) \rightarrow\left(\mathscr{D}^{-1}\right)^{6}(L(i)) \circ\left(\mathscr{D}^{-1}\right)^{6}\left(M_{0}\right) .
$$

By the induction hypothesis, $\left(\mathscr{D}^{-1}\right)^{6}(f)$ is isomorphic to

$$
\left(M_{0} \circ L(i) \rightarrow L(i) \circ M_{0}\right) \circ \mathrm{C}_{\beta+w_{0} \beta}
$$

Since it is non-zero, the uniqueness property of $f$ implies that it is equal to $f \circ C_{\beta+w_{0} \beta}$ up to a constant multiple. Hence its image is isomorphic to $M \circ \mathrm{C}_{\beta+w_{0} \beta}$.

Conjecture 5.15. We can choose isomorphism (5.2) functorially in $M \in \widetilde{\mathscr{C}}_{w_{0}}$. 


\section{REFERENCES}

[1] M. Auslander, and I. Reiten, DTr-periodic modules and functors, Representation theory of algebra (Cocoyoc, 1994), CMS Conf. Proc., vol. 18, Amer. Math. Soc., Providence, RI, 1996, pp. 39-50.

[2] A. Berenstein, S. Fomin, and A. Zelevinsky, Parametrizations of canonical bases and totally positive matrices, Adv. Math. 122 (1996), no. 1, 49-149.

[3] A. Berenstein and D. Rupel, Quantum cluster characters of Hall algebras, Selecta Math. (N.S.) 21 (2015), no. 4, 1121-1176.

[4] A. Berenstein and A. Zelevinsky, Total positivity in Schubert varieties, Comment. Math.Helv. 72 (1997), no. 1, 128-166.

[5] _ Q Quantum cluster algebras, Adv. Math. 195 (2005), no. 2, 405-455.

[6] K. Erdmann and N. Snashall, Preprojective algebras of Dynkin type, periodicity and the second Hochschild cohomology, Algebras and modules, II (Geiranger, 1996), CMS Conf. Proc., vol. 24, Amer. Math. Soc., Providence, RI, 1998, pp. 183-193.

[7] J. Hong and S.-J. Kang, Introduction to Quantum Groups and Crystal Bases, Graduate Studies in Mathematics, 42. American Mathematical Society, Providence, RI, 2002.

[8] C. Geiß, B. Leclerc and J. Schröer, Generic bases for cluster algebras and the Chamber Ansatz, J. Amer. Math. Soc. 25 (2012), no. 1, 21-76.

[9] _ Cluster structures on quantum coordinate rings, Selecta Math. (N.S.) 19 (2013), no. 2, $337-397$.

[10] K. R. Goodearl and M. T. Yakimov, The Berenstein-Zelevinsky quantum cluster algebra conjecture, J. Eur. Math. Soc. (JEMS) 22 (2020), no. 8, 2453-2509.

[11] S.-J. Kang and M. Kashiwara, Categorification of Highest Weight Modules via Khovanov-LaudaRouquier Algebras, Invent. Math. 190 (2012), no. 3, 699-742.

[12] S.-J. Kang, M. Kashiwara and M. Kim, Symmetric quiver Hecke algebras and R-matrices of quantum affine algebras, Invent. Math. 211 (2018), no. 2, 591-685.

[13] S.-J. Kang, M. Kashiwara, M. Kim and S.-j. Oh, Simplicity of heads and socles of tensor products, Compos. Math. 151 (2015), no. 2, 377-396.

[14] _ Monoidal categorification of cluster algebras, J. Amer. Math. Soc. 31 (2018), no. 2, 349-426.

[15] M. Kashiwara, M. Kim, S.-j. Oh, and E. Park, Monoidal categories associated with strata of flag manifolds, Adv. Math. 328 (2018), 959-1009.

[16] M. Kashiwara and E. Park, Affinizations and R-matrices for quiver Hecke algebras, J. Eur. Math. Soc. 20, 1161-1193.

[17] M. Kashiwara and P. Schapira, Categories and Sheaves, Grundlehren der Mathematischen Wissenschaften, vol. 332, Springer-Verlag, Berlin (2006).

[18] M. Khovanov and A. Lauda, A diagrammatic approach to categorification of quantum groups I, Represent. Theory 13 (2009), 309-347.

[19] _ A diagrammatic approach to categorification of quantum groups II, Trans. Amer. Math. Soc. 363 (2011), 2685-2700.

[20] Y. Kimura and H. Oya, Twist automorphisms on quantum unipotent cells and dual canonical bases, Internat. Math. Research Notices (IMRN), (2019) (DOI: https://doi.org/10.1093/imrn/rnz040.) 
[21] F. Qin, Triangular bases in quantum cluster algebras and monoidal categorification conjectures, Duke Math. 166 (2017), 2337-2442.

[22] R. Rouquier, 2-Kac-Moody algebras, arXiv:0812.5023v1.

[23] — Quiver Hecke algebras and 2-Lie algebras, Algebra Colloq. 19 (2012), no. 2, 359-410.

[24] P. Tingley and B. Webster, Mirković-Vilonen polytopes and Khovanov-Lauda-Roquuier algebras, Compos. Math. 152 (2016), no. 8, 1648-1696.

[25] M. Varagnolo and E. Vasserot, Canonical bases and KLR algebras, J. Reine Angew. Math. 659 (2011), 67-100.

(Masaki Kashiwara) Kyoto University Institute for Advanced Study, Research Institute for Mathematical Sciences, Kyoto University, Kyoto 606-8502, Japan \& Korea Institute for Advanced Study, Seoul 02455, Korea

Email address, Masaki Kashiwara: masaki@kurims.kyoto-u.ac.jp

(Myungho Kim) Department of Mathematics, Kyung Hee University, Seoul 02447, KoreA

Email address, Myungho Kim: mkim@khu.ac.kr

(Se-jin Oh) Department of Mathematics, Ewha Womans University, Seoul 120-750, KOREA

Email address, Se-jin Oh: sejin092@gmail.com

(Euiyong Park) Department of Mathematics, University of Seoul, Seoul 02504, Korea

Email address, Euiyong Park: epark@uos.ac.kr 